\title{
Selenocyanation of Aryl and Styryl methyl ketones in presence of Selenium dioxide and Malononitrile: An approach for the synthesis of $\alpha$-Carbonyl Selenocyanates
}

\author{
Ibakyntiew D. Marpna, Kmendashisha Wanniang, Tyrchain Mitre Lipon, O. Risuklang \\ Shangpliang and Bekington Myrboh* \\ *Department of Chemistry, North-Eastern Hill University, Shillong, India, 793022 \\ Correspondence: bmyrboh@nehu.ac.in
}

\section{Supporting Information}

1. X-Ray diffraction Data S2- S3

2. ${ }^{1} \mathrm{HNMR}$ and ${ }^{13} \mathrm{CNMR}$ Data S4- S59 


\section{Single-Crystal X-Ray data for compound 3a}

The crystallographic data of compound 3a was recorded using Bruker D8 VENTURE SCXRD using a Mo K- $\alpha$ radiation source $(\lambda=0.71073 \AA)$ equipped with PHOTON 100 CMOS detector source with a highly accurate goniometer. The crystal was kept at 293(2) K during data collection. Using Olex $2,{ }^{1}$ the structure was solved with the ShelXT ${ }^{2}$ structure solution program using Intrinsic Phasing and refined with the ShelXL ${ }^{3}$ refinement package using Least Squares minimisation.

1. Dolomanov, O.V., Bourhis, L.J., Gildea, R.J, Howard, J.A.K. \& Puschmann, H. (2009), J. Appl. Cryst. 42, 339-341.

2. Sheldrick, G.M. (2015). Acta Cryst. A71, 3-8.

3. Sheldrick, G.M. (2015). Acta Cryst. C71, 3-8.

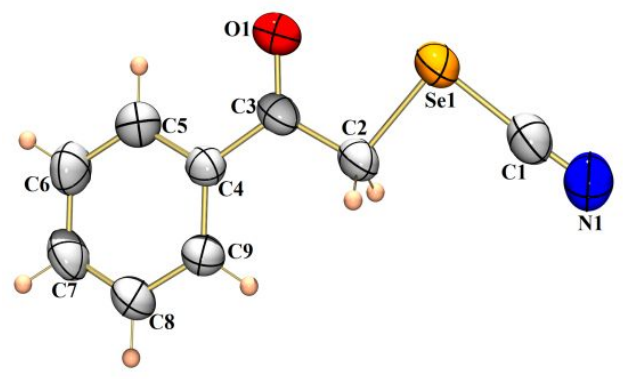

Figure S1. Ortep Diagram of Compound 3a (CCDC 2041476) with 50\% ellipsoid contour

Table 1 Crystal data and structure refinement for $3 a$.

Identification code

1

Empirical formula

$\mathrm{C}_{9} \mathrm{H}_{7} \mathrm{NOSe}$

Formula weight

224.12

Temperature/K

293(2)

Crystal system

monoclinic

Space group

$\mathrm{P} 2{ }_{1} / \mathrm{c}$

$\begin{array}{ll}\mathrm{a} / \AA & 10.7317(10) \\ \mathrm{b} / \AA & 5.7101(4) \\ \mathrm{c} / \AA & 15.3249(12) \\ \alpha /{ }^{\circ} & 90 \\ \beta /{ }^{\circ} & 106.032(3) \\ \gamma /{ }^{\circ} & 90 \\ \mathrm{Volume} / \AA^{3} & 902.57(13) \\ \mathrm{Z} & 4 \\ \rho_{\text {calcg }} / \mathrm{cm}^{3} & 1.649 \\ \mu / \mathrm{mm}^{-1} & 4.108\end{array}$


$\mathrm{F}(000)$

440.0

Crystal size $/ \mathrm{mm}^{3}$

$0.18 \times 0.15 \times 0.15$

Radiation

$\operatorname{MoK} \alpha(\lambda=0.71073)$

$2 \Theta$ range for data collection $/{ }^{\circ} 5.842$ to 59.758

Index ranges

$-13 \leq \mathrm{h} \leq 14,-7 \leq \mathrm{k} \leq 7,-20 \leq 1 \leq 21$

Reflections collected

19003

Independent reflections

$2226\left[\mathrm{R}_{\text {int }}=0.0628, \mathrm{R}_{\text {sigma }}=0.0387\right]$

Data/restraints/parameters

$2226 / 0 / 109$

Goodness-of-fit on $\mathrm{F}^{2}$

1.087

Final $R$ indexes $[\mathrm{I}>=2 \sigma(\mathrm{I})] \quad \mathrm{R}_{1}=0.0778, \mathrm{wR}_{2}=0.2186$

Final $\mathrm{R}$ indexes [all data] $\quad \mathrm{R}_{1}=0.1250, \mathrm{wR}_{2}=0.2660$

Largest diff. peak/hole / e $\AA^{-3} 0.56 /-0.92$ 
${ }^{1} \mathrm{H}$ NMR (400 MHz, $\mathrm{CDCl}_{3}$ ) spectrum of 1-phenyl-2-selenocyanatoethan-1-one (3a)
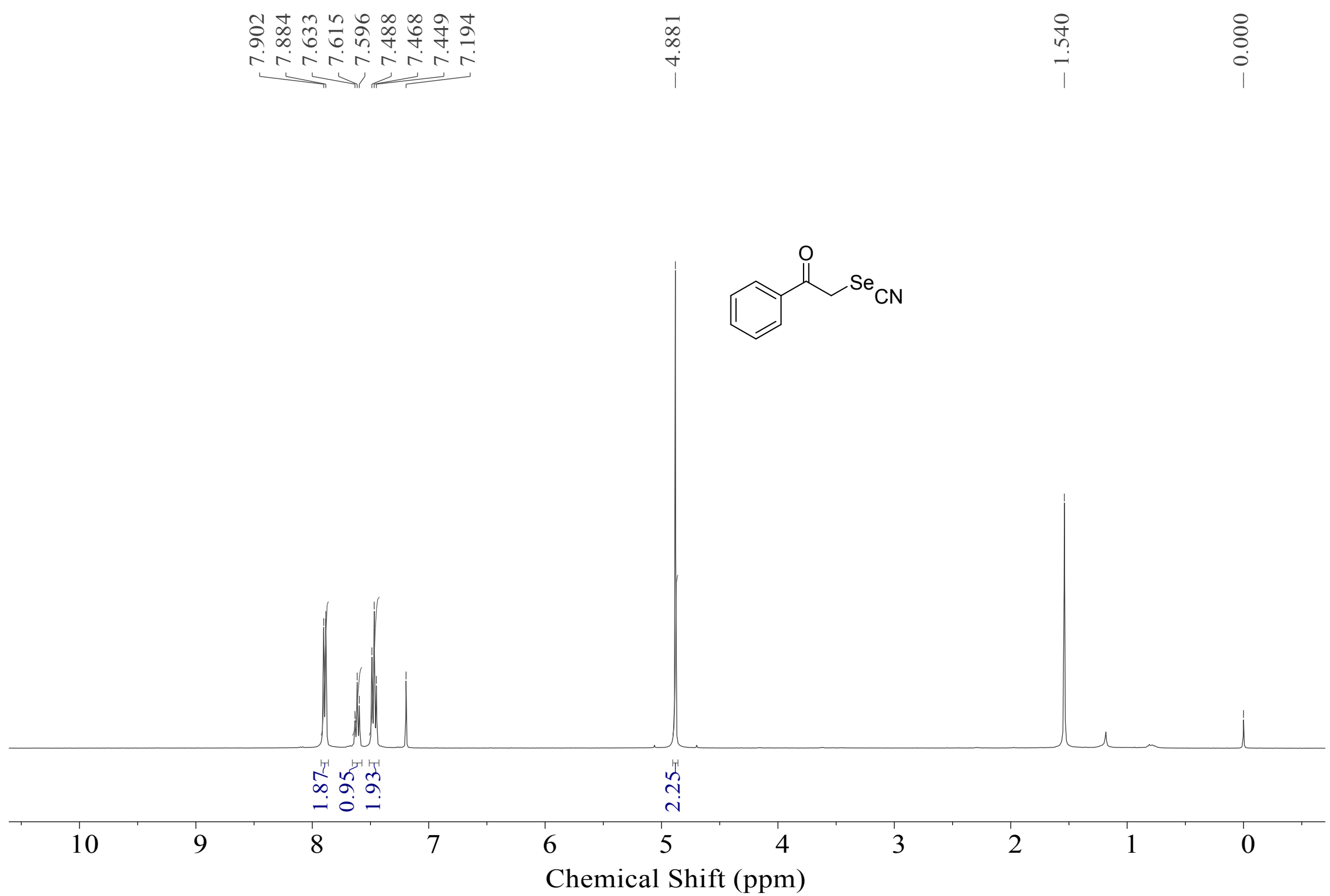
${ }^{13} \mathrm{C}\left\{{ }^{1} \mathrm{H}\right\}$ NMR (100 MHz, $\left.\mathrm{CDCl}_{3}\right)$ spectrum of 1-phenyl-2-selenocyanatoethan-1-one (3a)

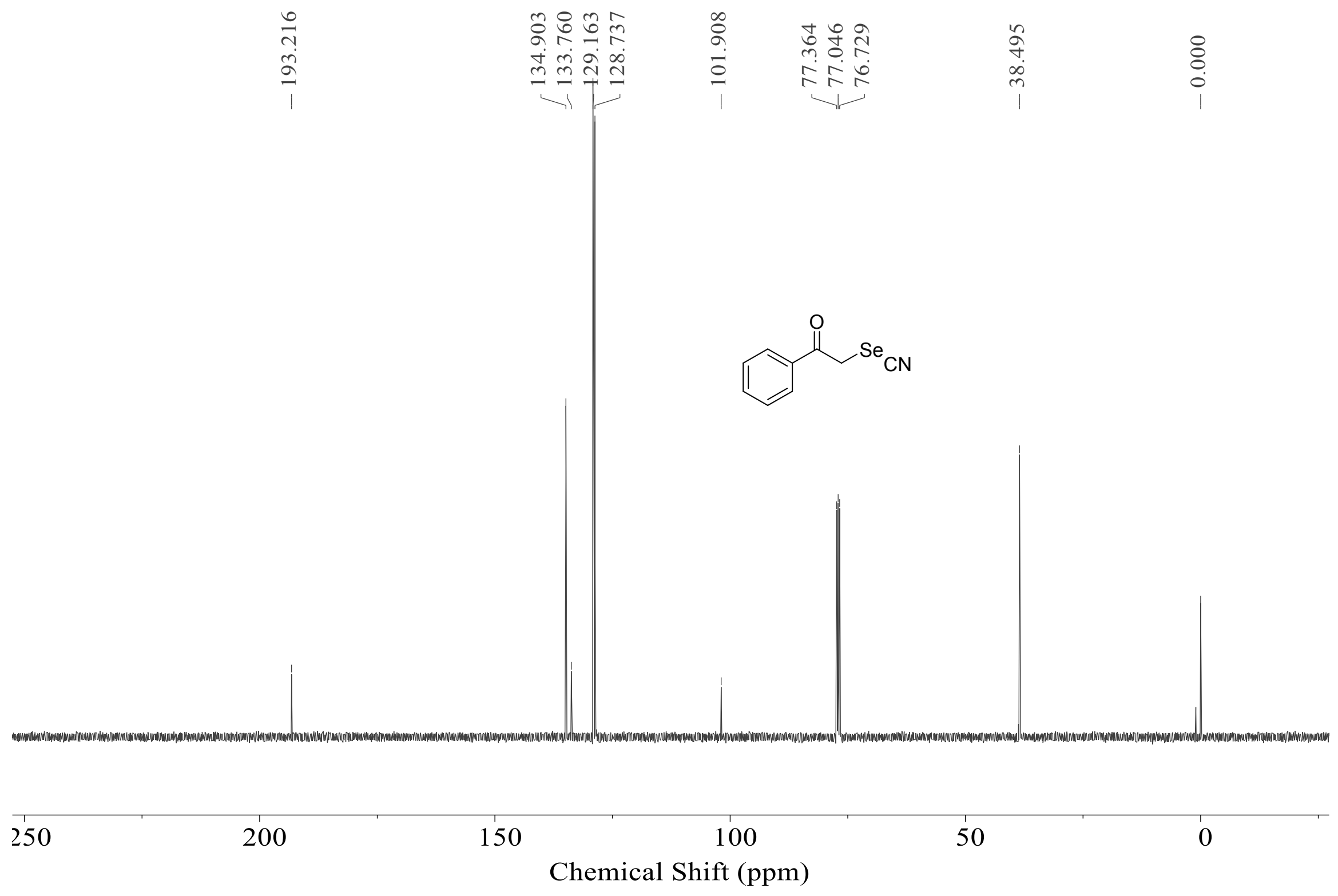


${ }^{1} \mathrm{H}$ NMR (400 MHz, $\mathrm{CDCl}_{3}$ ) spectrum of 2-selenocyanato-1-(p-tolyl)ethan-1-one (3b)

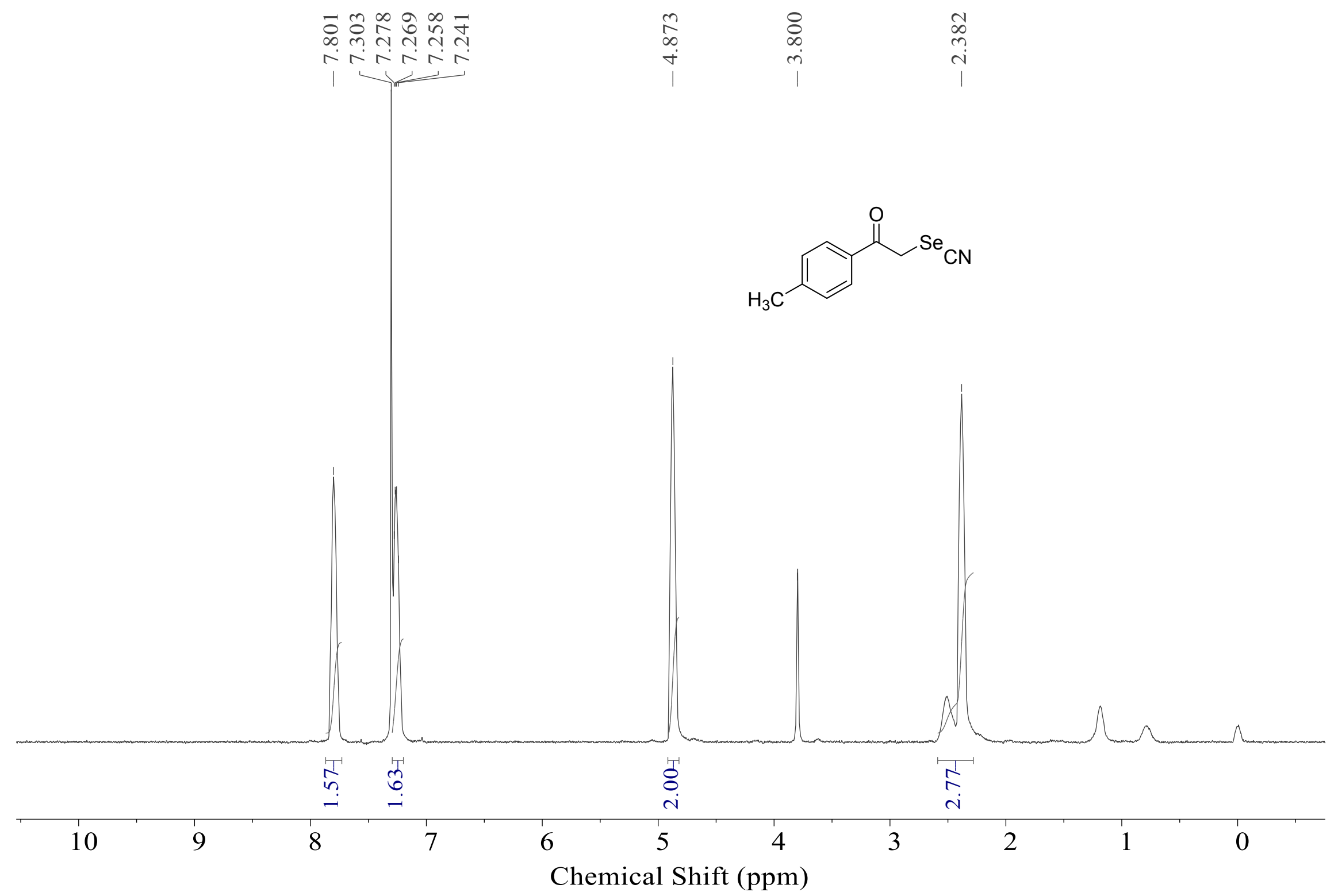


${ }^{13} \mathrm{C}\left\{{ }^{1} \mathrm{H}\right\}$ NMR $\left(100 \mathrm{MHz}, \mathrm{CDCl}_{3}\right)$ spectrum of 2-selenocyanato-1-(p-tolyl)ethan-1-one (3b)

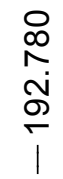

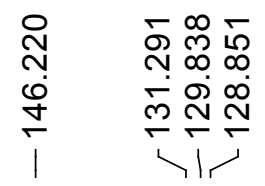

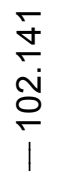

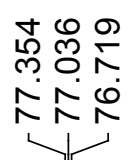

0
0
0
0
0
0

ֻீ

8
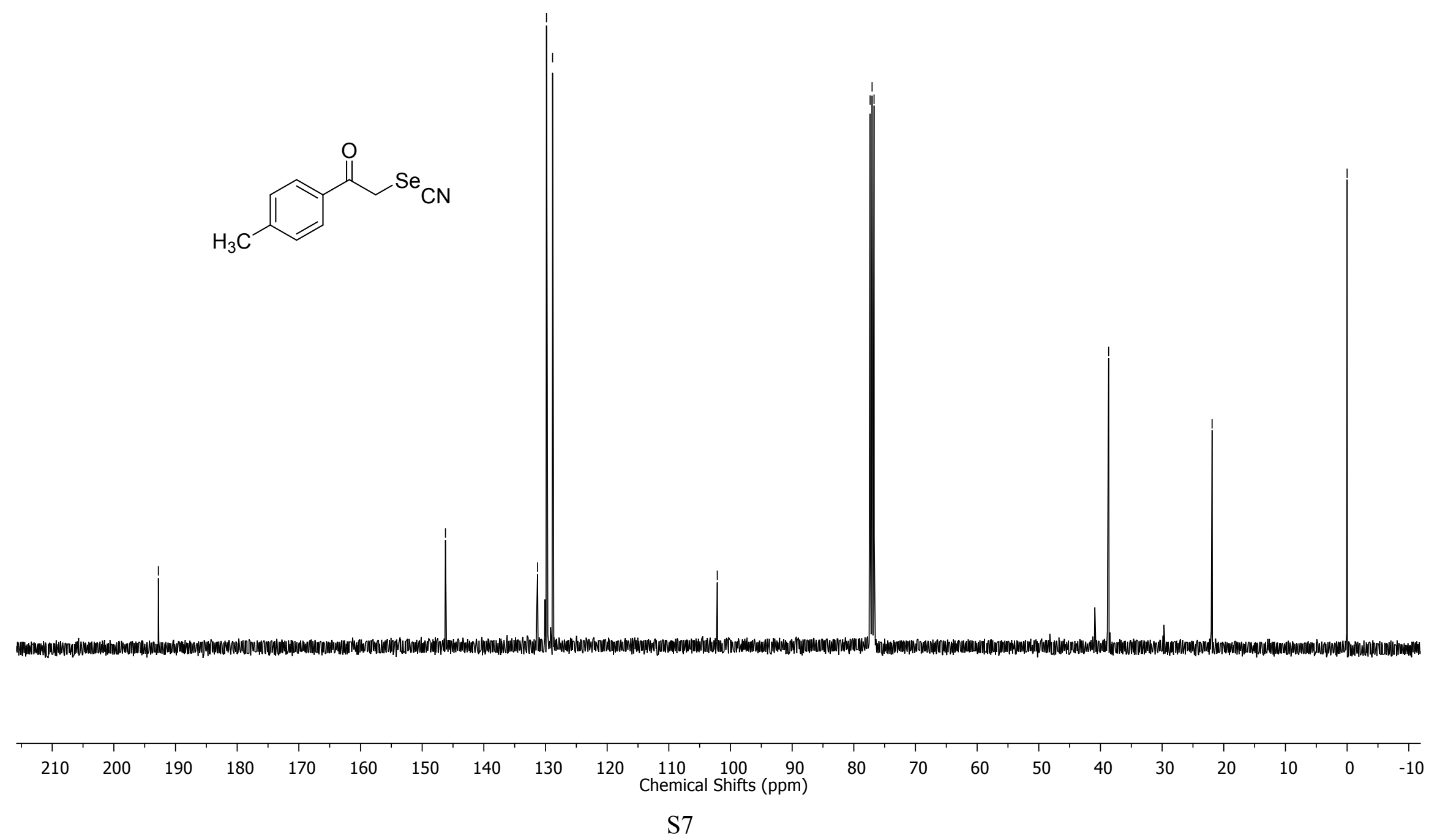
${ }^{1} \mathrm{H}$ NMR (400 MHz, $\mathrm{CDCl}_{3}$ ) spectrum of 1-(4-methoxyphenyl)-2-selenocyanatoethan-1-one (3c)

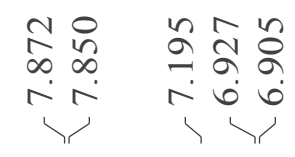

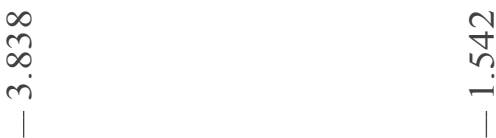
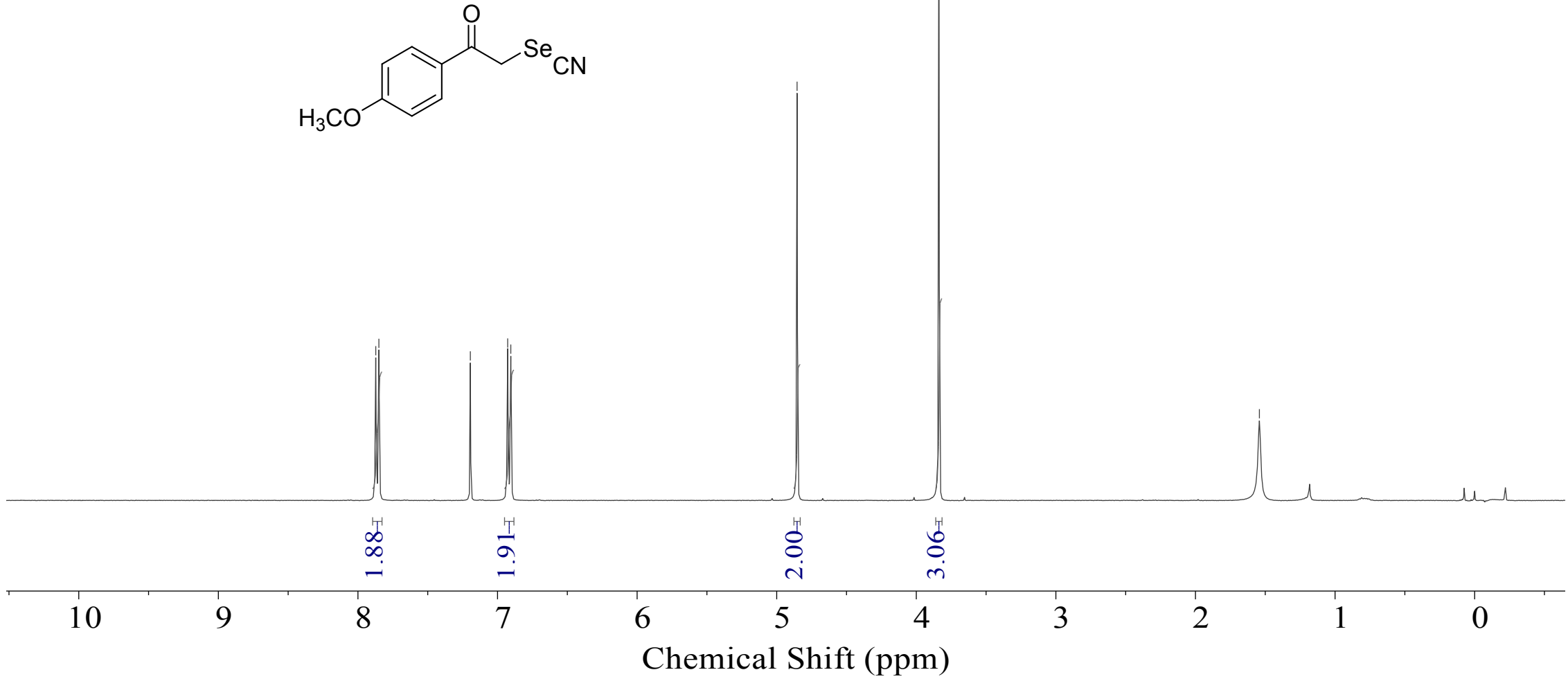
${ }^{13} \mathrm{C}\left\{{ }^{1} \mathrm{H}\right\}$ NMR (100 MHz, $\left.\mathrm{CDCl}_{3}\right)$ spectrum of 1-(4-methoxyphenyl)-2-selenocyanatoethan-1-one (3c)

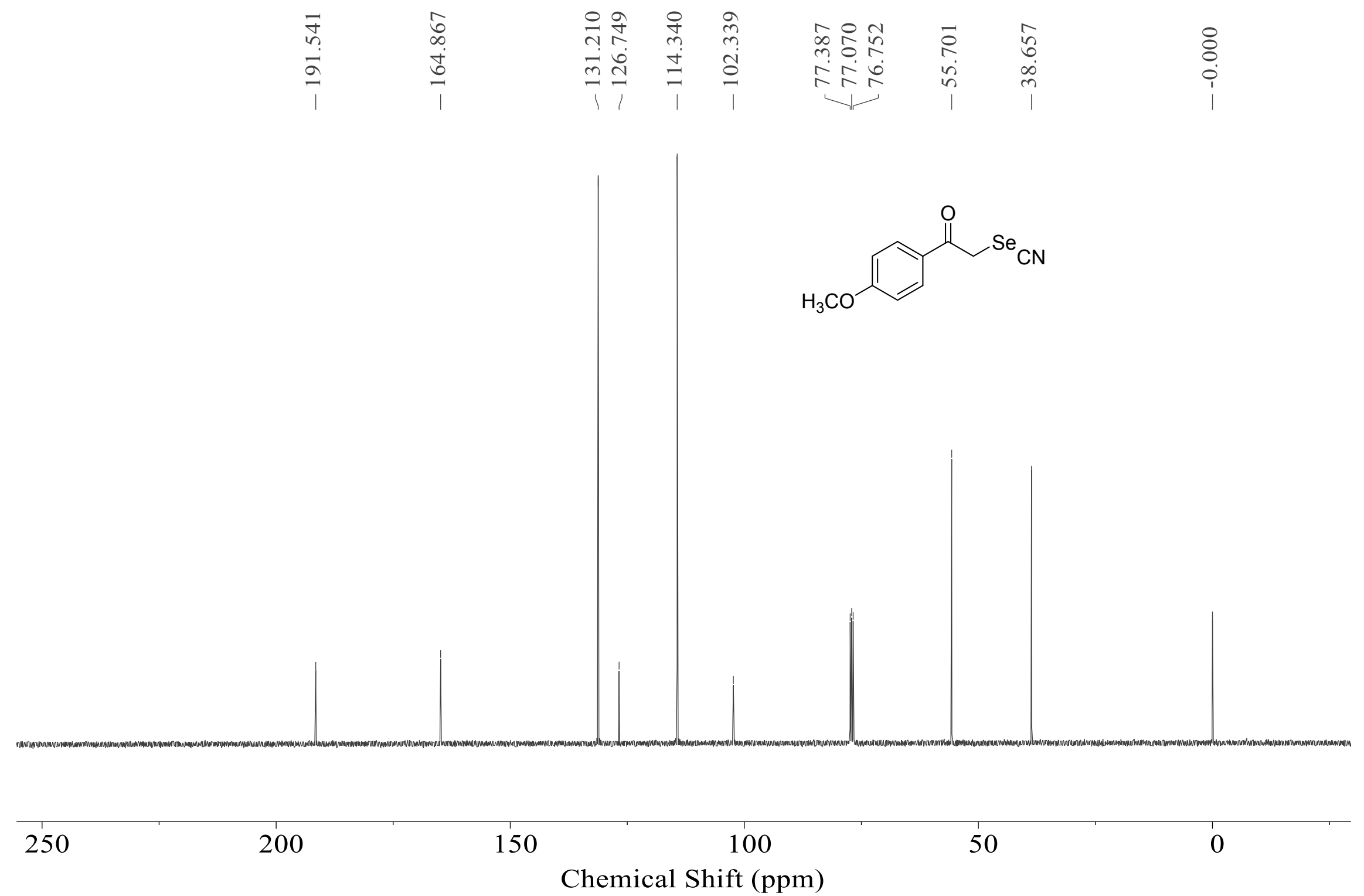


${ }^{1} \mathrm{H}$ NMR (400 MHz, $\mathrm{CDCl}_{3}$ ) spectrum of 1-(4-nitrophenyl)-2-selenocyanatoethan-1-one (3d)

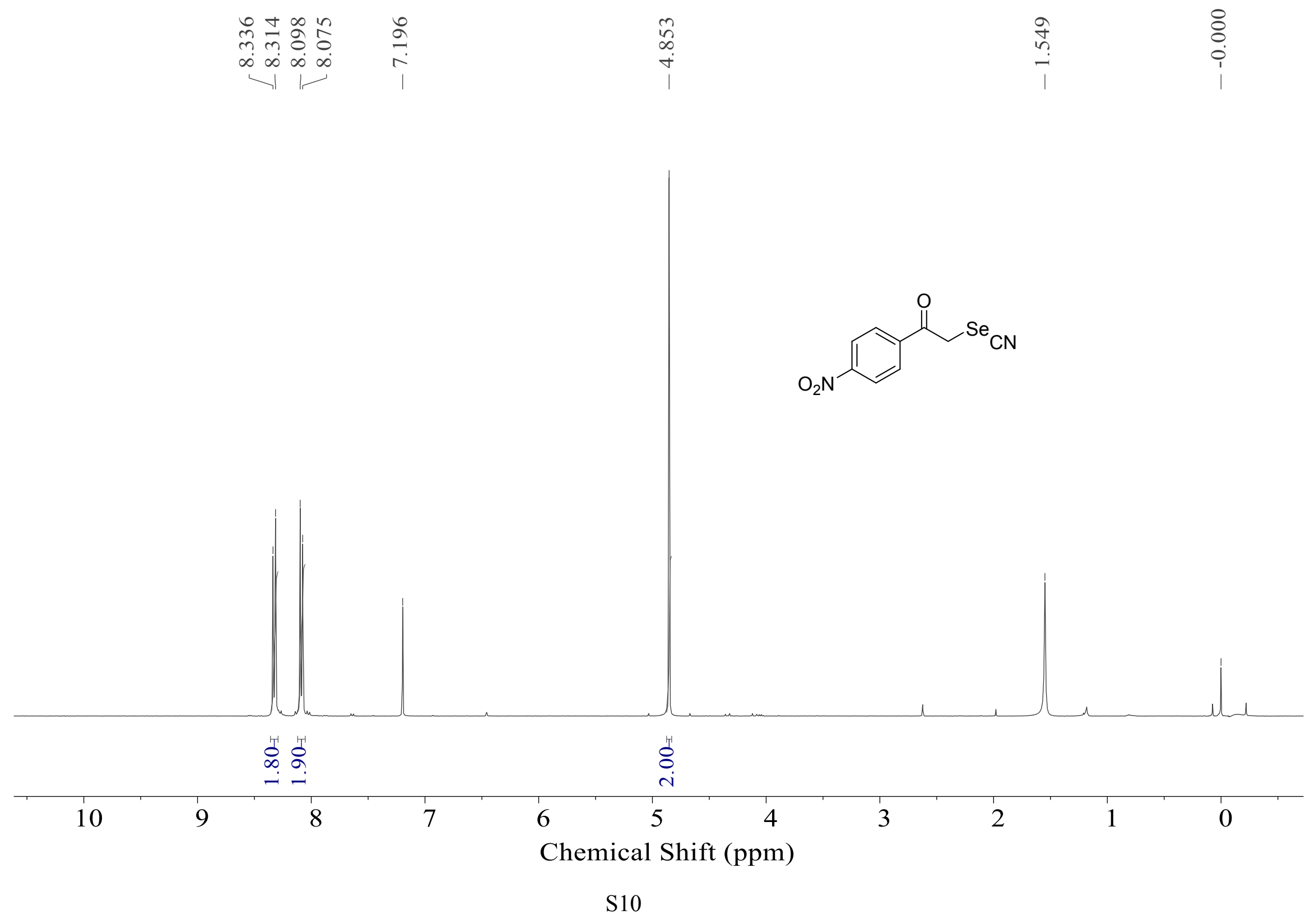


${ }^{13} \mathrm{C}\left\{{ }^{1} \mathrm{H}\right\}$ NMR (100 MHz, $\left.\mathrm{CDCl}_{3}\right)$ spectrum of 1-(4-nitrophenyl)-2-selenocyanatoethan-1-one (3d)

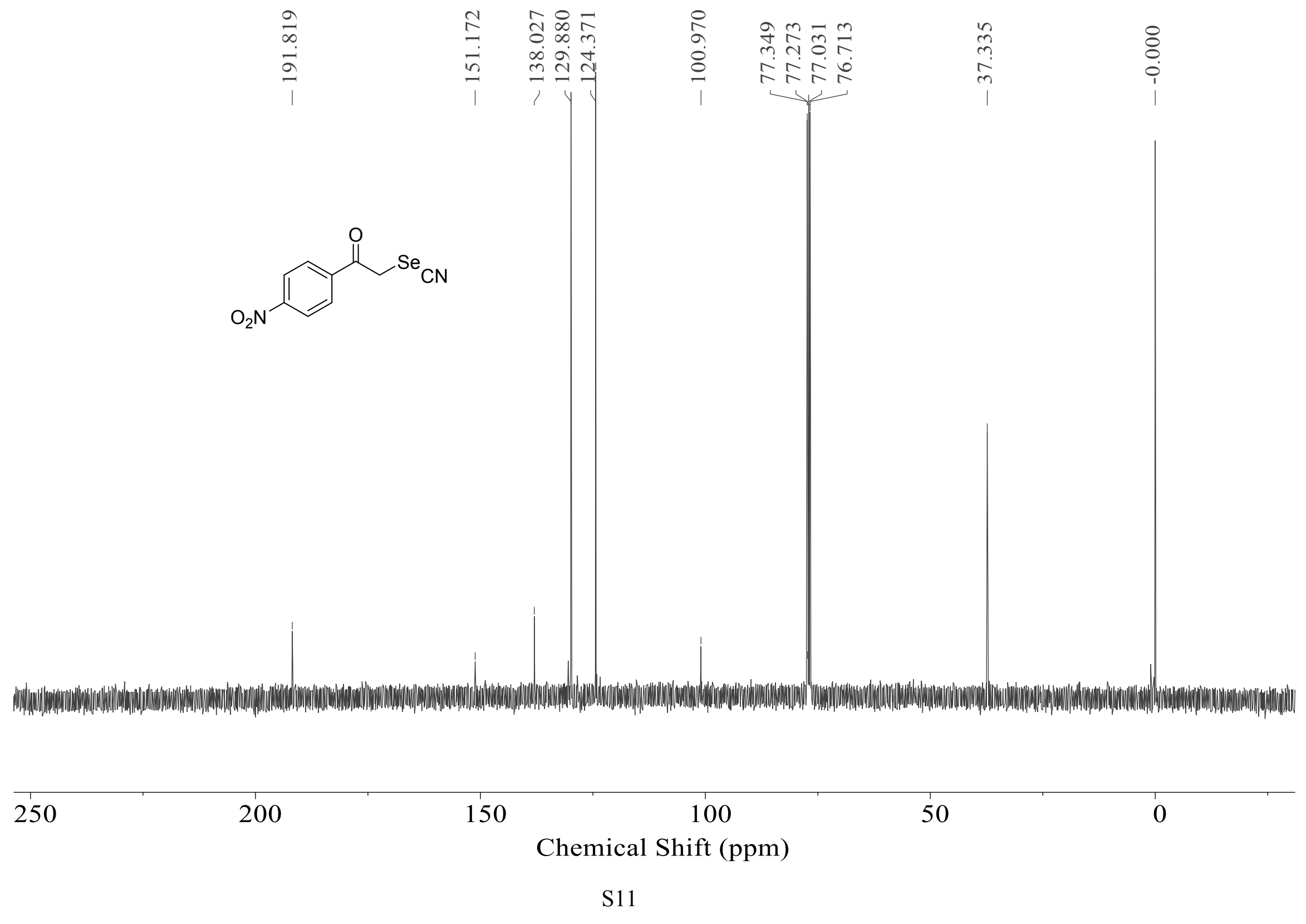


${ }^{1} \mathrm{H}$ NMR (400 MHz, $\mathrm{CDCl}_{3}$ ) spectrum of 1-(4-chlorophenyl)-2-selenocyanatoethan-1-one (3e)

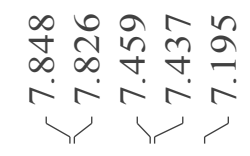

$\vec{\infty}$
$\infty$
$\dot{\sigma}$
1

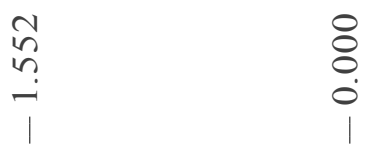
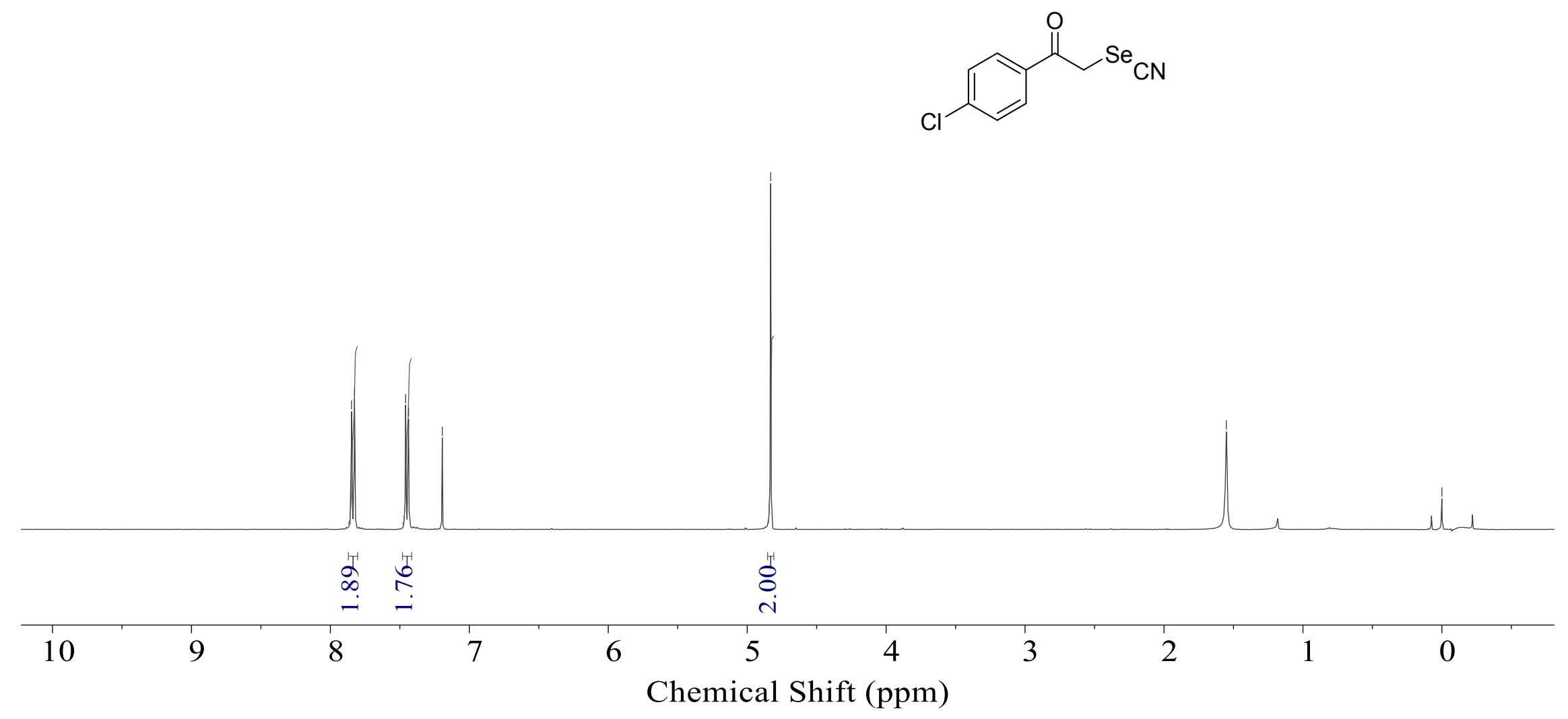
${ }^{13} \mathrm{C}\left\{{ }^{1} \mathrm{H}\right\}$ NMR (100 MHz, $\left.\mathrm{CDCl}_{3}\right)$ spectrum of 1-(4-chlorophenyl)-2-selenocyanatoethan-1-one (3e)

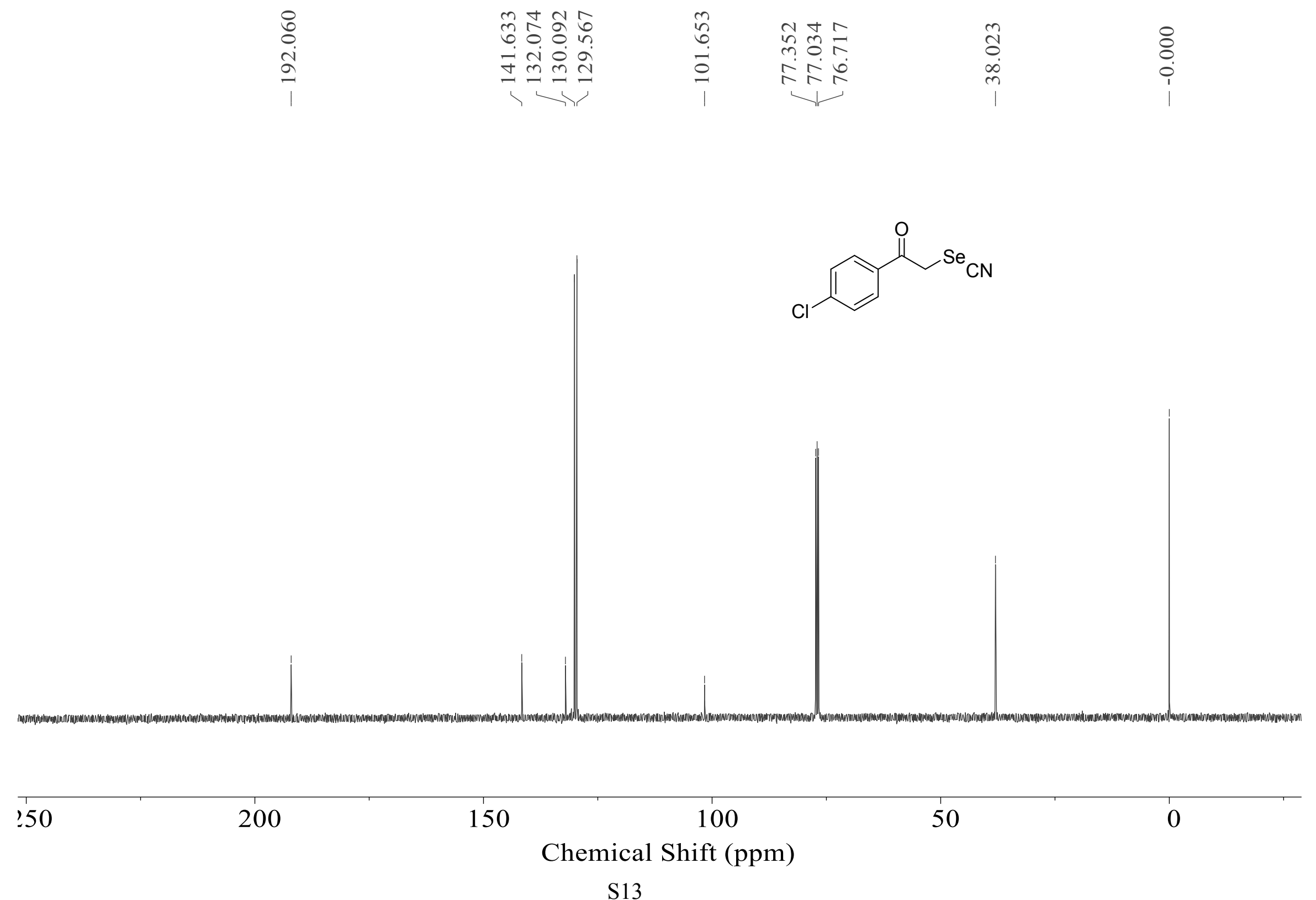


${ }^{1} \mathrm{H}$ NMR (400 MHz, $\mathrm{CDCl}_{3}$ ) spectrum of 1-(4-bromophenyl)-2-selenocyanatoethan-1-one (3f)

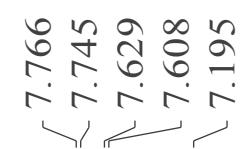

N
$\infty$
+

$\infty$
$n$
$n$
$\cdots$

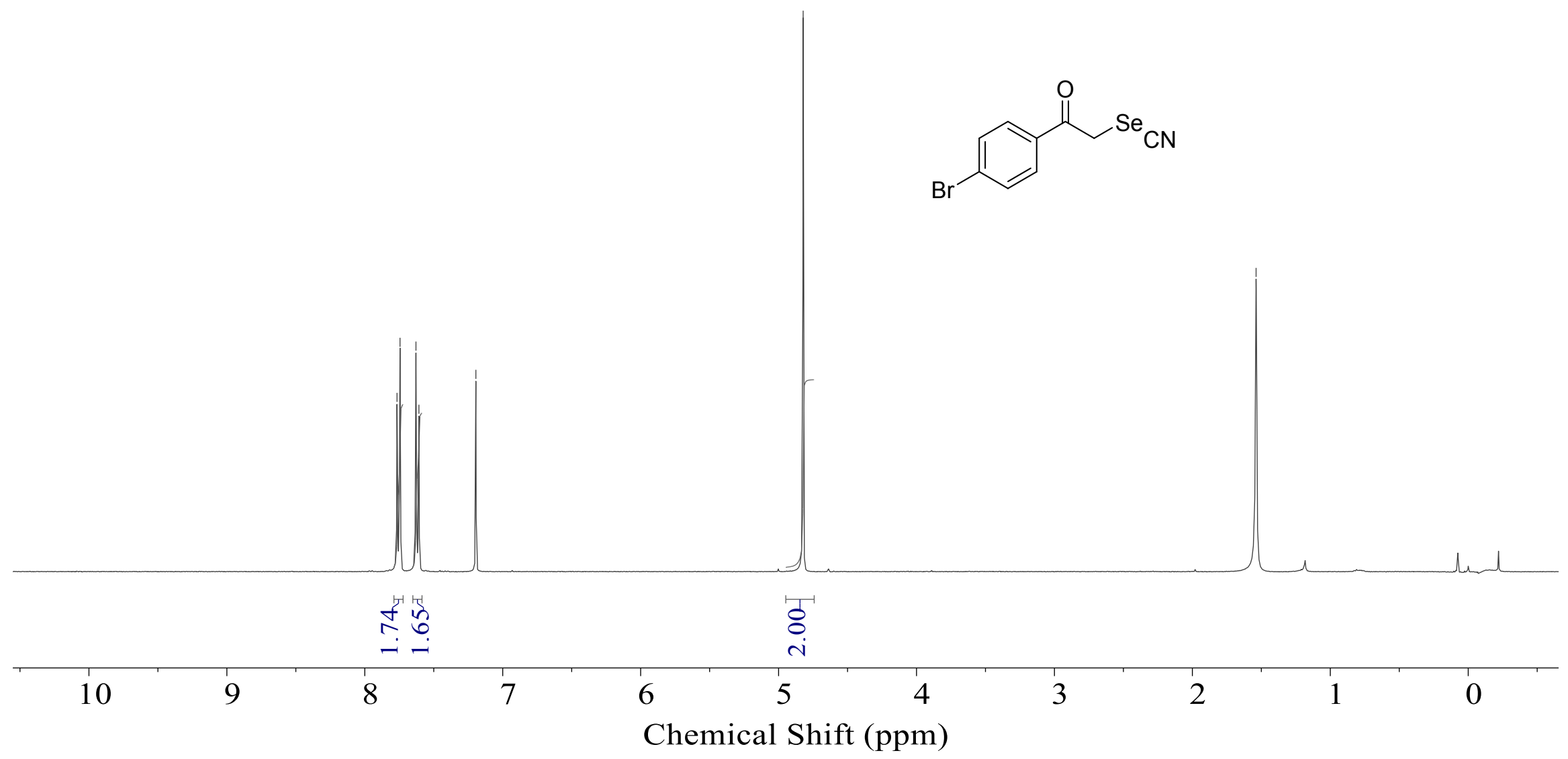


${ }^{13} \mathrm{C}\left\{{ }^{1} \mathrm{H}\right\}$ NMR (100 MHz, $\mathrm{CDCl}_{3}$ : DMSO- $\left.d_{6}\right)$ spectrum of 1-(4-bromophenyl)-2-selenocyanatoethan-1-one (3f)

\begin{tabular}{|c|c|c|c|c|}
\hline & 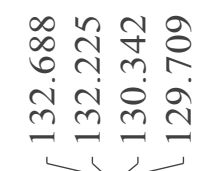 & 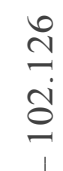 & 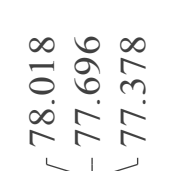 & 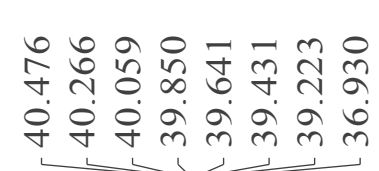 \\
\hline
\end{tabular}
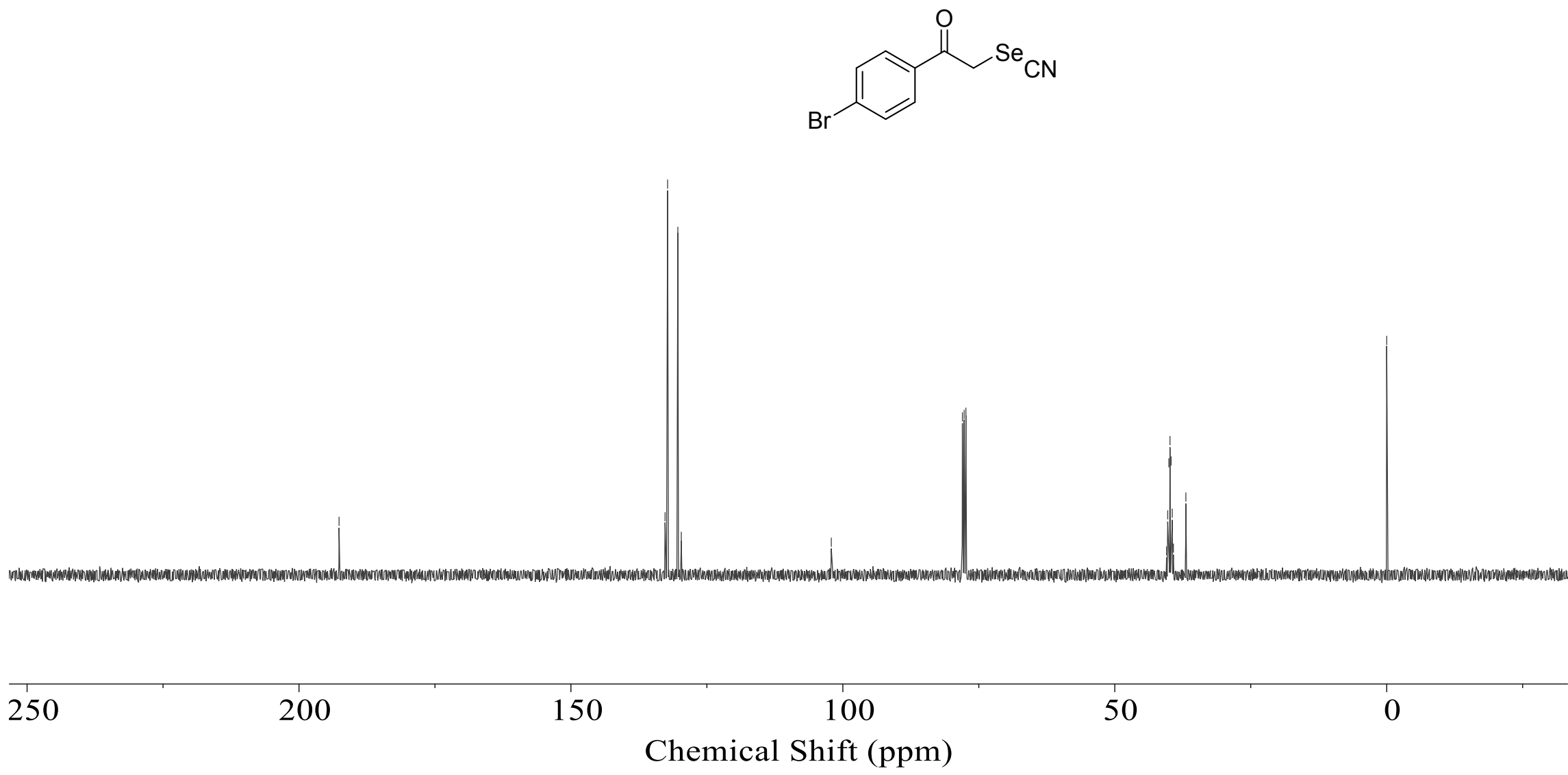
${ }^{1} \mathrm{H}$ NMR (400 MHz, $\mathrm{CDCl}_{3}$ ) spectrum of 1-(4-fluorophenyl)-2-selenocyanatoethan-1-one (3g)

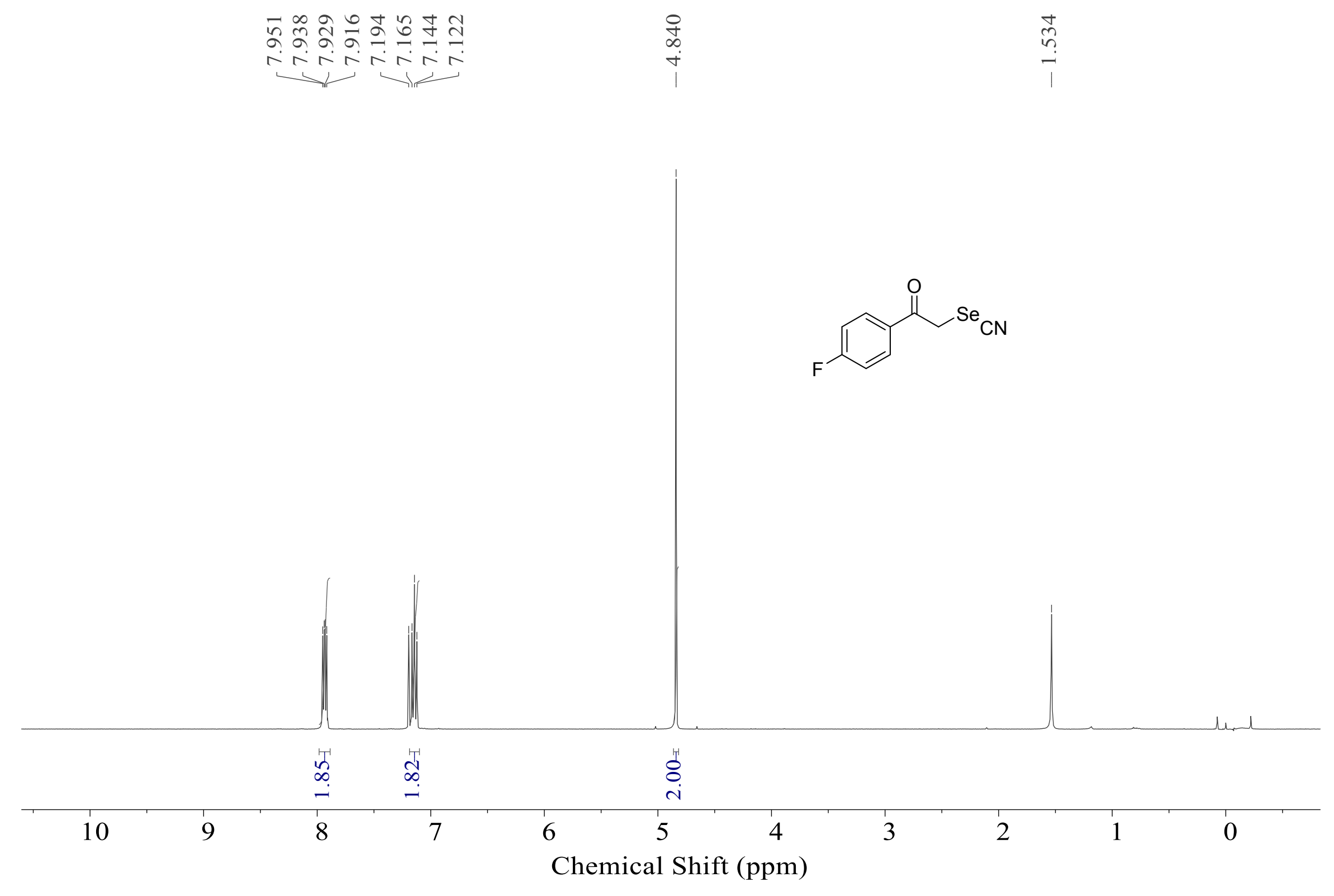


${ }^{13} \mathrm{C}\left\{{ }^{1} \mathrm{H}\right\} \mathrm{NMR}\left(100 \mathrm{MHz}, \mathrm{CDCl}_{3}\right)$ spectrum of 1-(4-fluorophenyl)-2-selenocyanatoethan-1-one (3g)

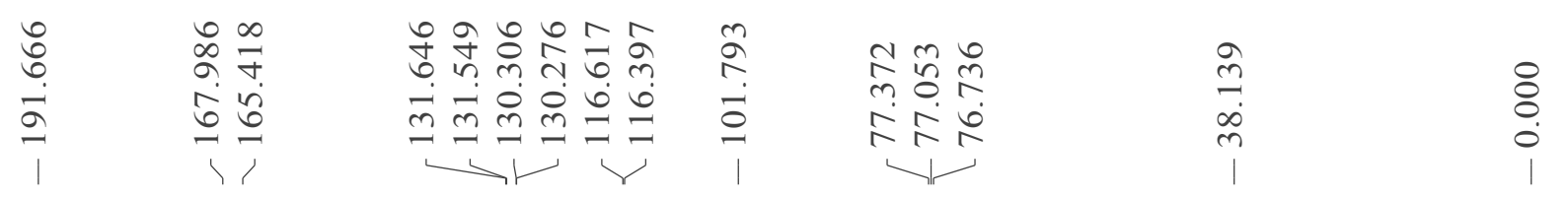
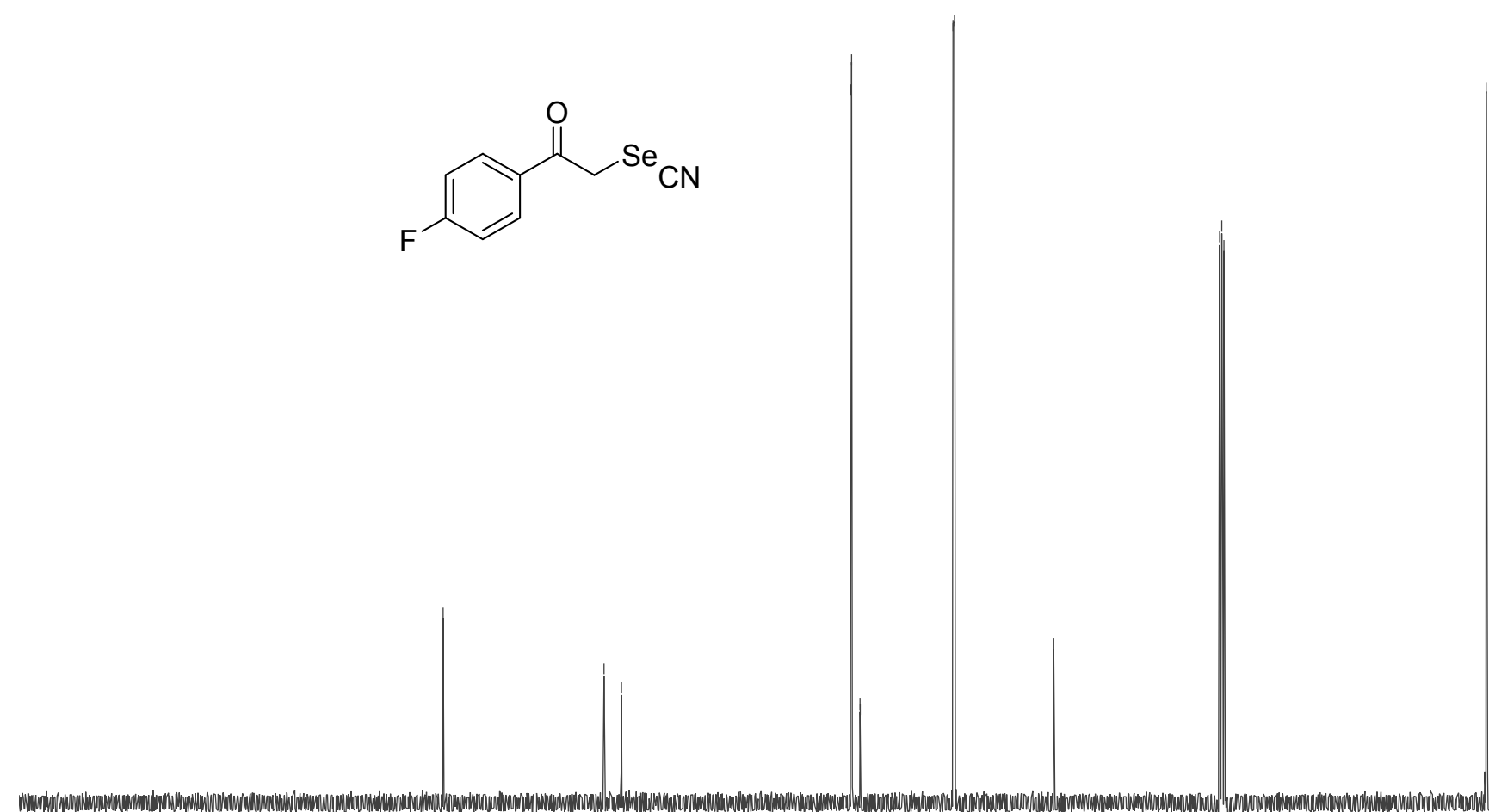

M.

\section{Chemical Shift (ppm)}


${ }^{1} \mathrm{H}$ NMR (400 MHz, $\mathrm{CDCl}_{3}$ ) spectrum of 1-(3-methoxyphenyl)-2-selenocyanatoethan-1-one (3h)

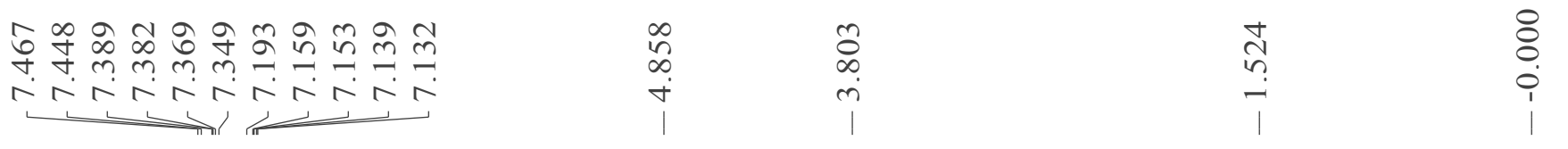

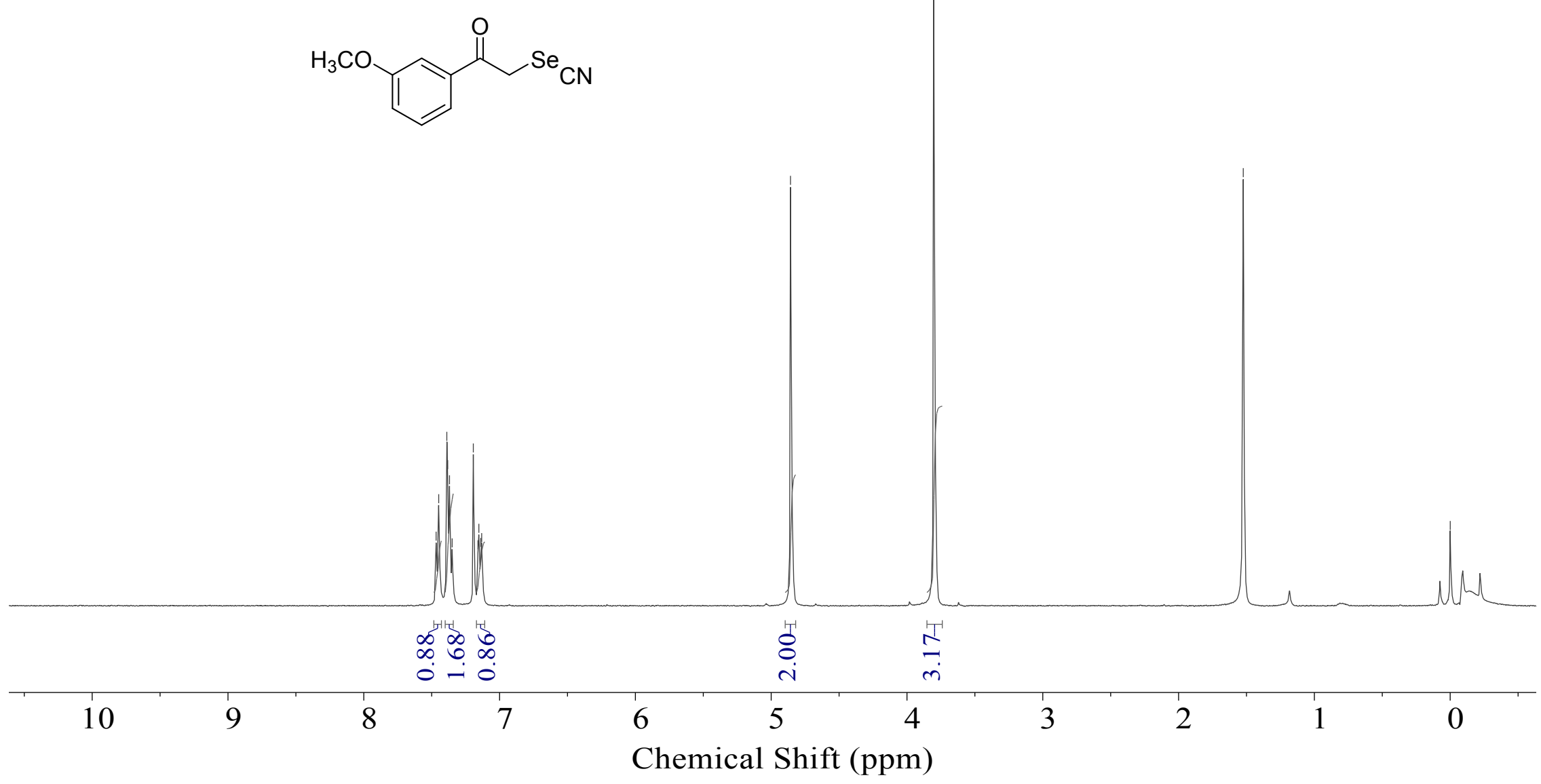


${ }^{13} \mathrm{C}\left\{{ }^{1} \mathrm{H}\right\}$ NMR (100 MHz, $\left.\mathrm{CDCl}_{3}\right)$ spectrum of 1-(3-methoxyphenyl)-2-selenocyanatoethan-1-one (3h)

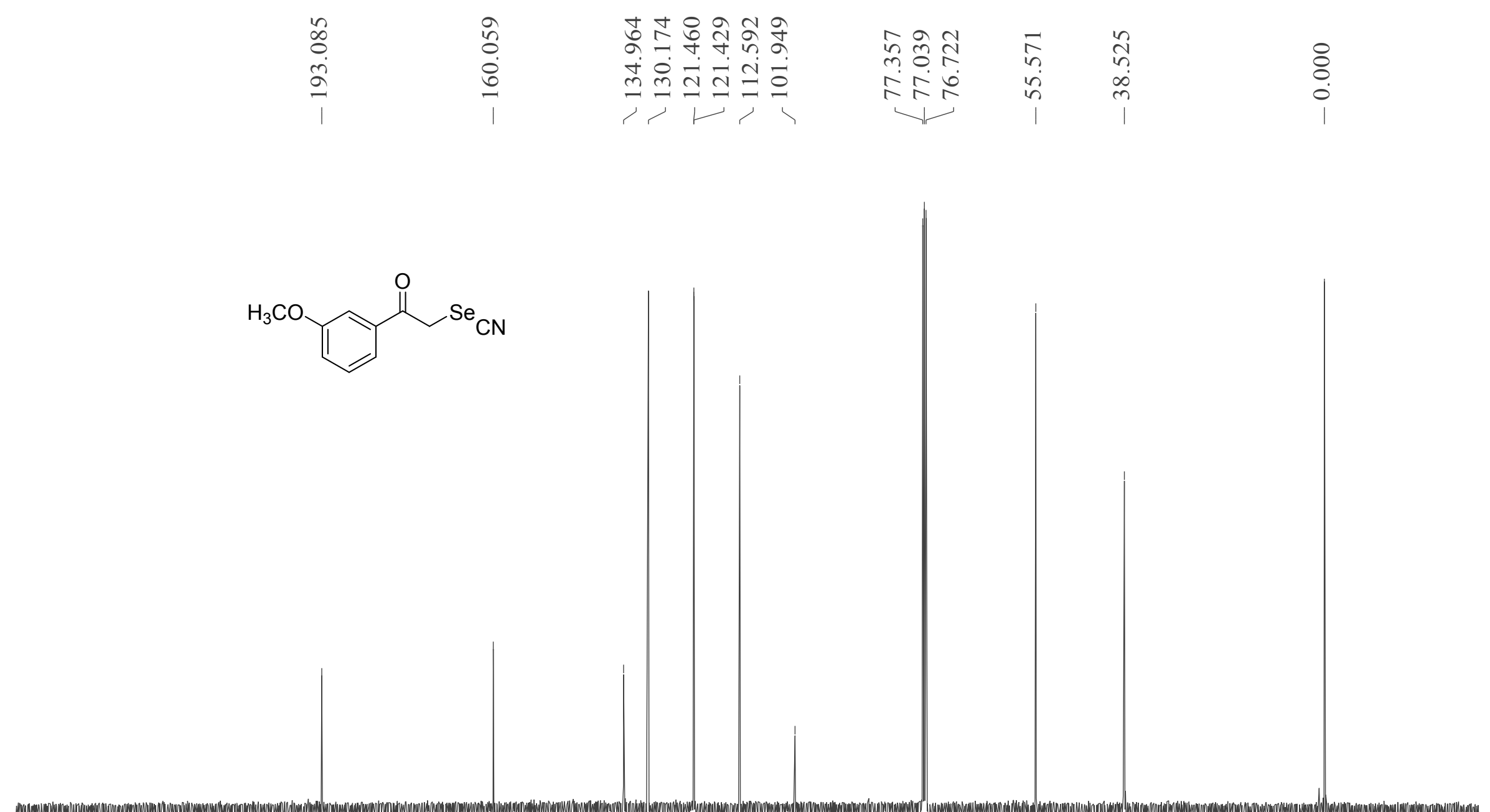

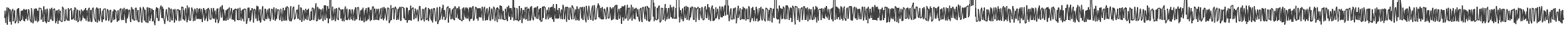

$? 50$

200

150

100

50

0

Chemical Shift (ppm) 
${ }^{1} \mathrm{H}$ NMR (400 MHz, $\mathrm{CDCl}_{3}$ ) spectrum of 1-(3-nitrophenyl)-2-selenocyanatoethan-1-one (3i)

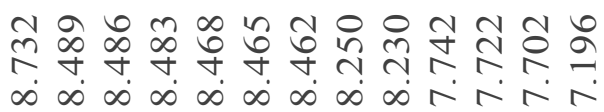

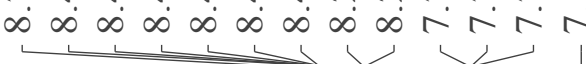
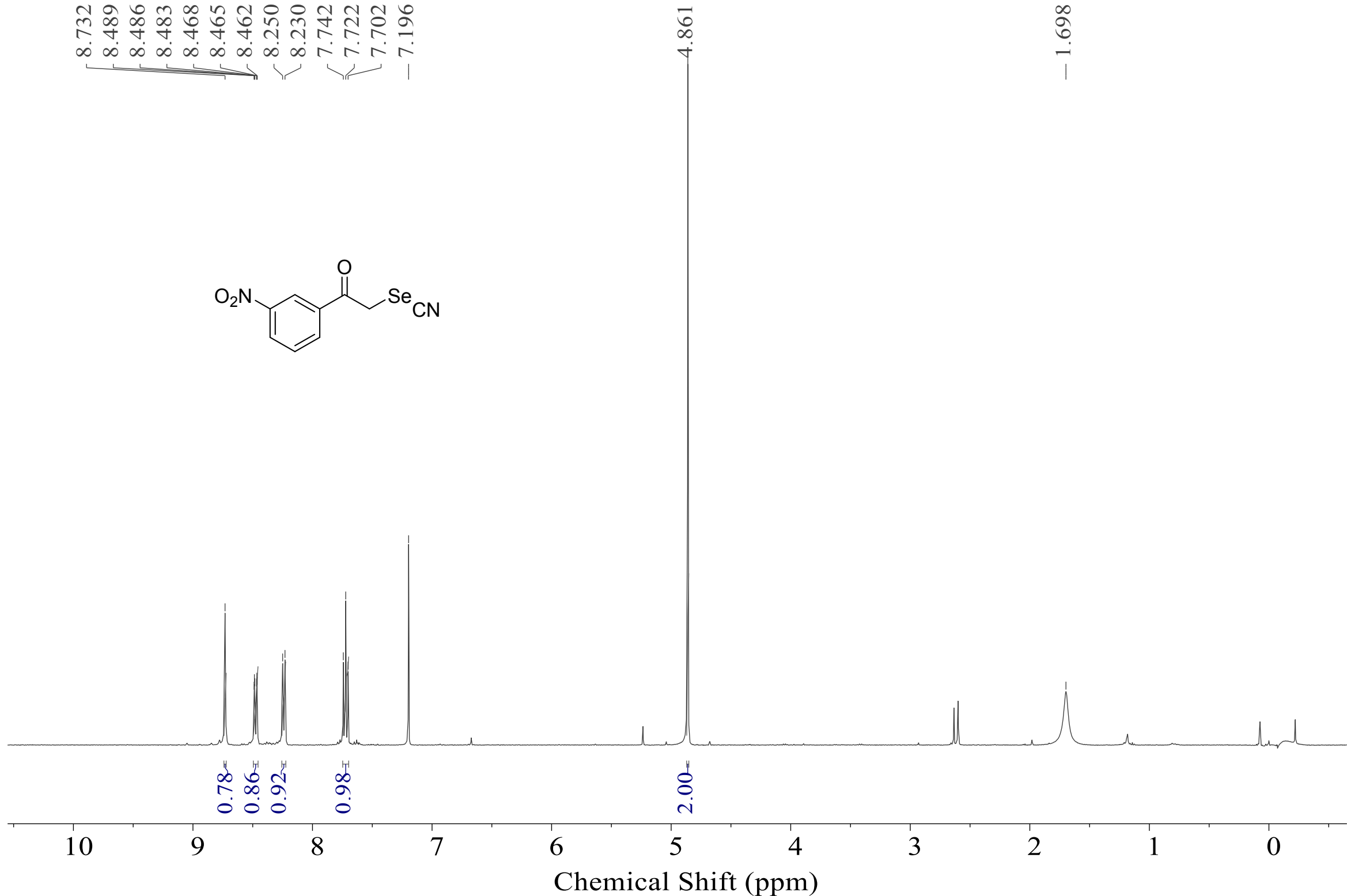
${ }^{13} \mathrm{C}\left\{{ }^{1} \mathrm{H}\right\}$ NMR (100 MHz, $\mathrm{CDCl}_{3}$ : DMSO- $\left.d_{6}\right)$ spectrum of 1-(3-nitrophenyl)-2-selenocyanatoethan-1-one (3i)

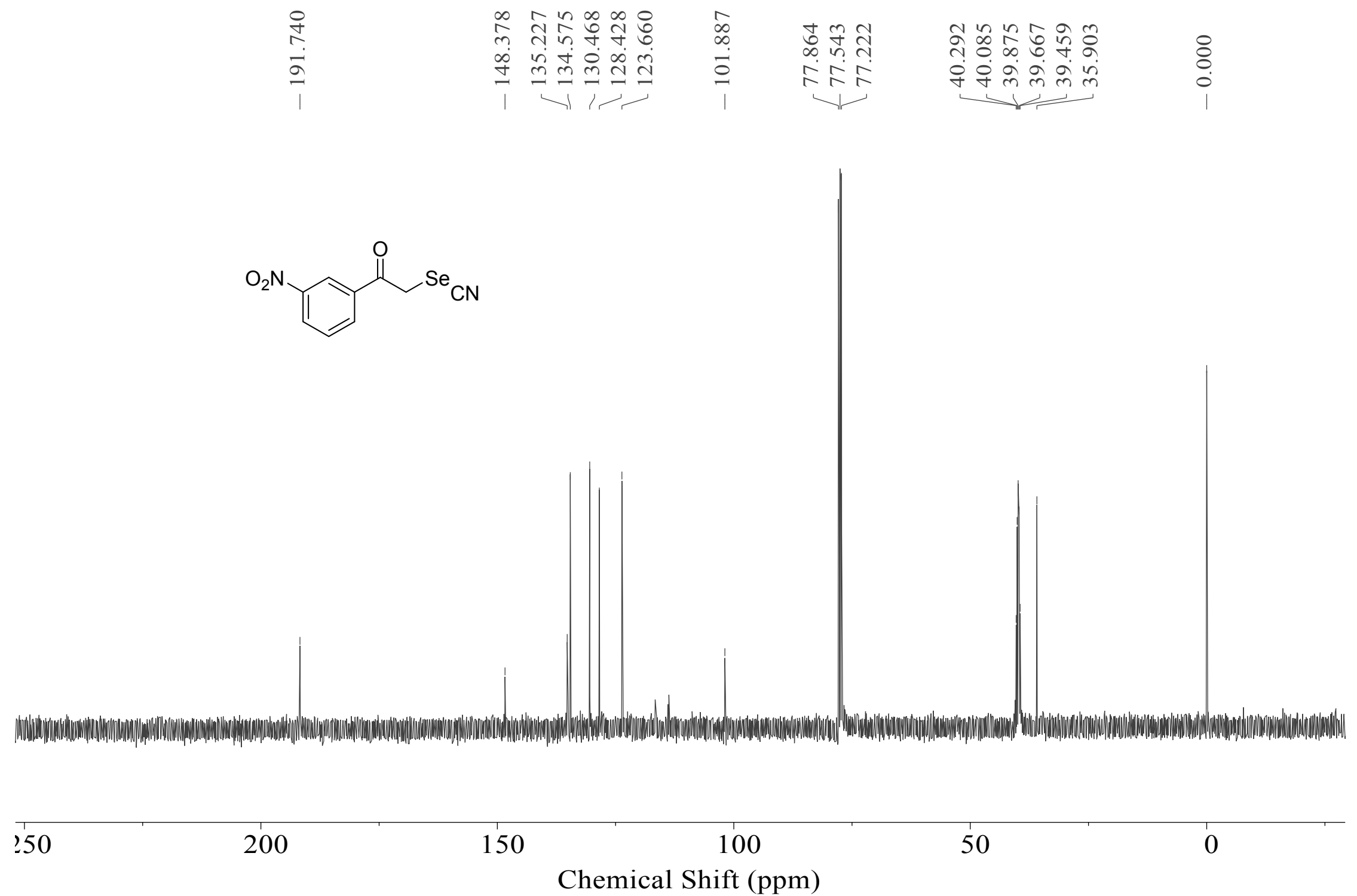


${ }^{1} \mathrm{H}$ NMR (400 MHz, $\mathrm{CDCl}_{3}$ ) spectrum of 1-(3-chlorophenyl)-2-selenocyanatoethan-1-one (3j)
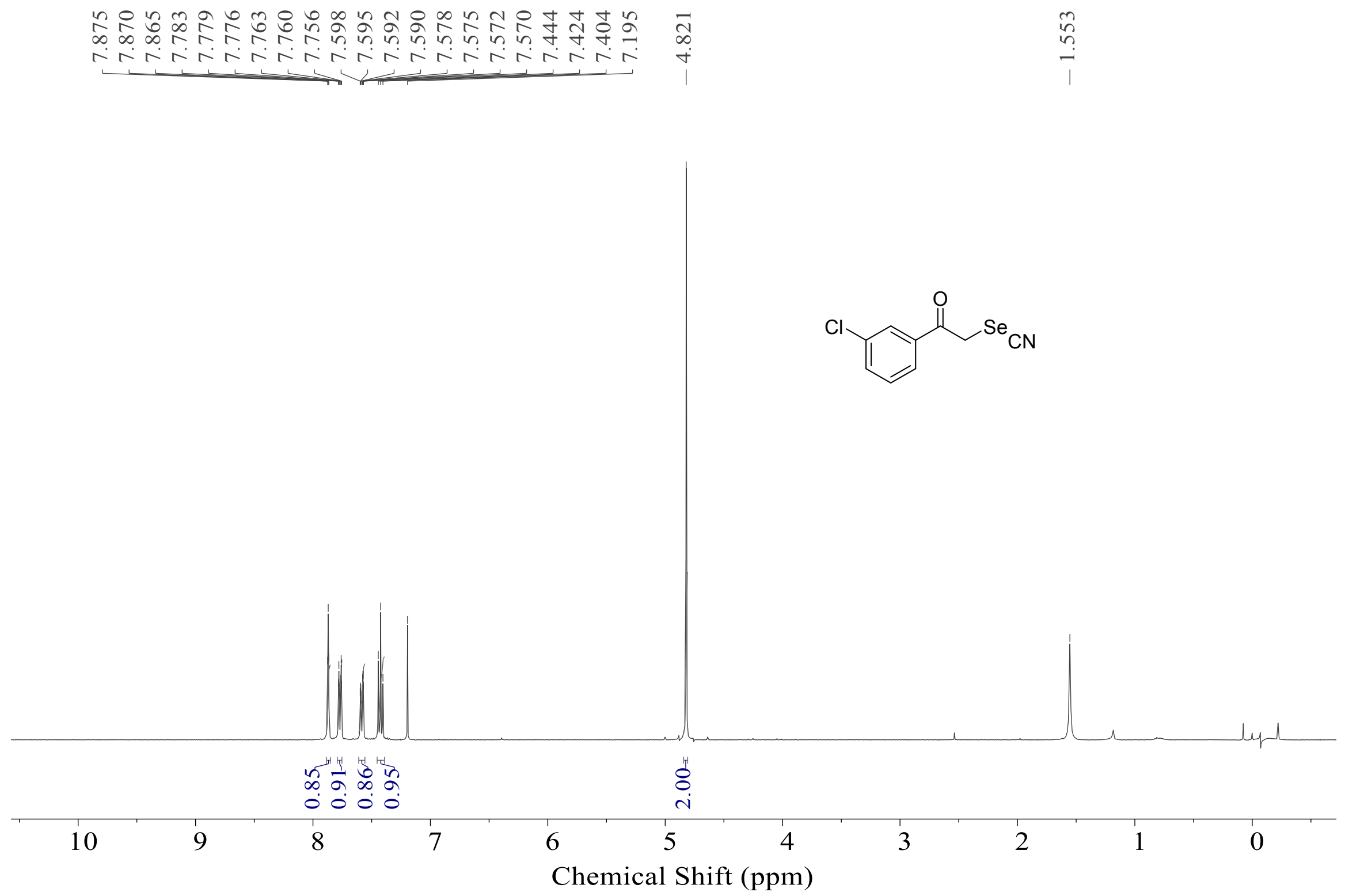
${ }^{13} \mathrm{C}\left\{{ }^{1} \mathrm{H}\right\}$ NMR (100 MHz, $\left.\mathrm{CDCl}_{3}\right)$ spectrum of 1-(3-chlorophenyl)-2-selenocyanatoethan-1-one (3j)

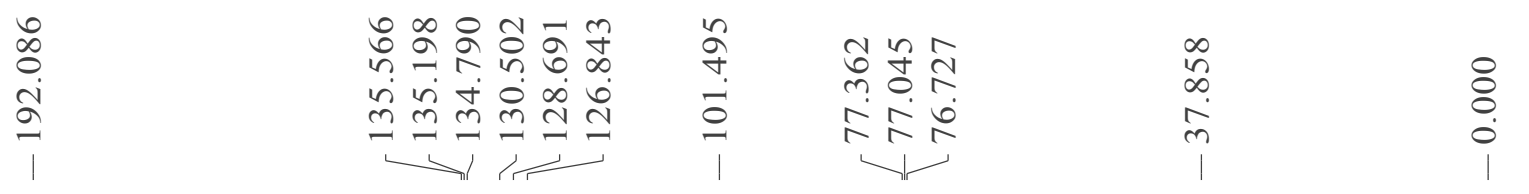<smiles>N#[13C]CC(=O)c1cccc(Cl)c1</smiles>

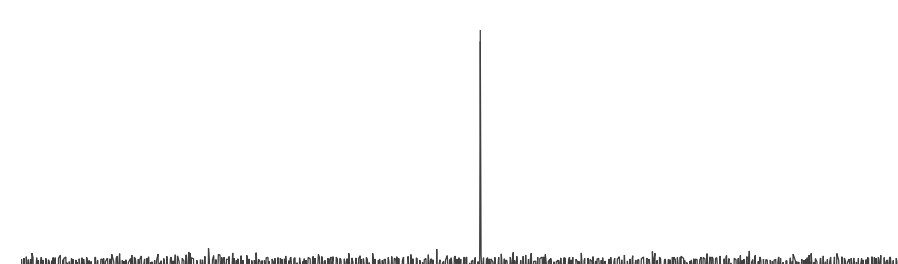
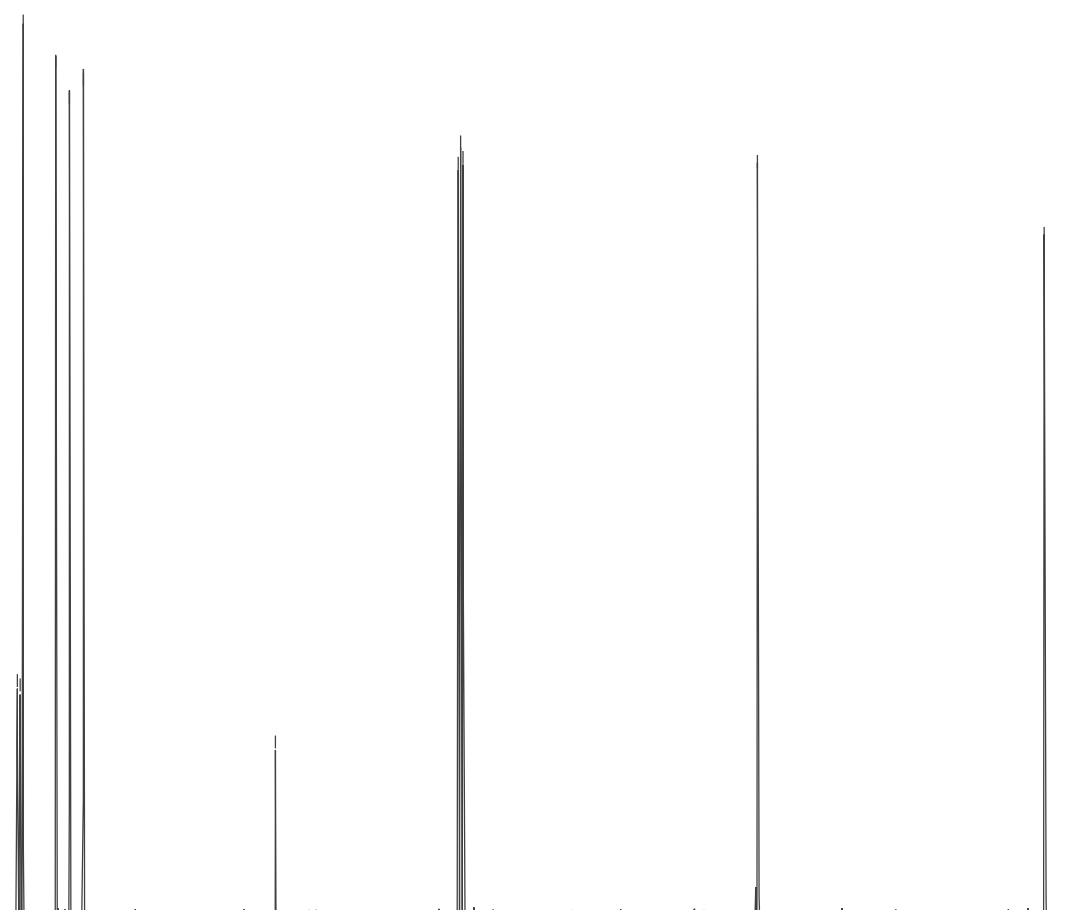

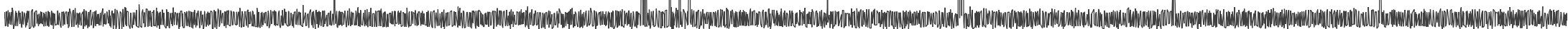


${ }^{1} \mathrm{H}$ NMR (400 MHz, $\mathrm{CDCl}_{3}$ ) spectrum of 1-(2-methoxyphenyl)-2-selenocyanatoethan-1-one (3k)

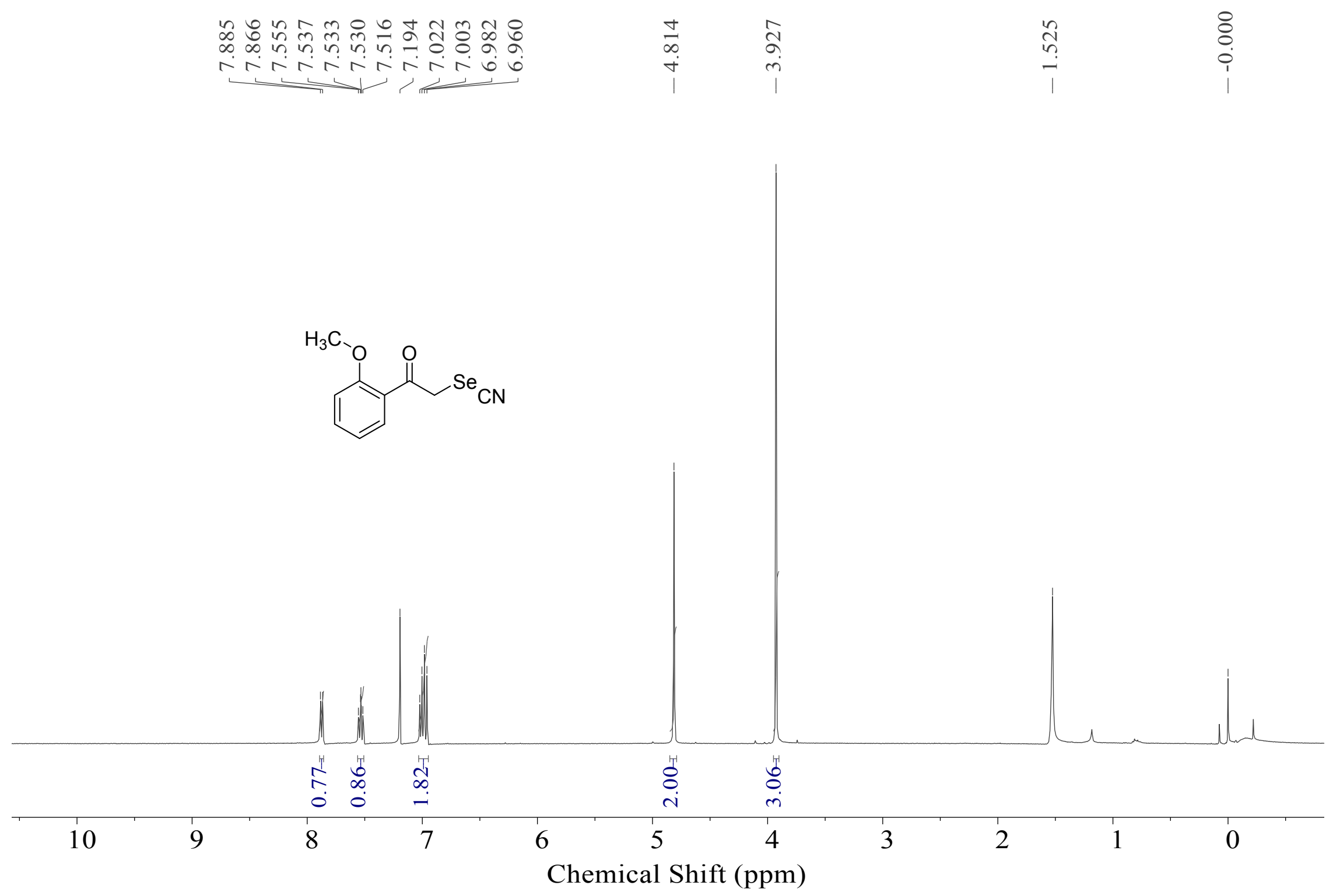


${ }^{13} \mathrm{C}\left\{{ }^{1} \mathrm{H}\right\} \mathrm{NMR}\left(100 \mathrm{MHz}, \mathrm{CDCl}_{3}\right)$ spectrum of 1-(2-methoxyphenyl)-2-selenocyanatoethan-1-one (3k)

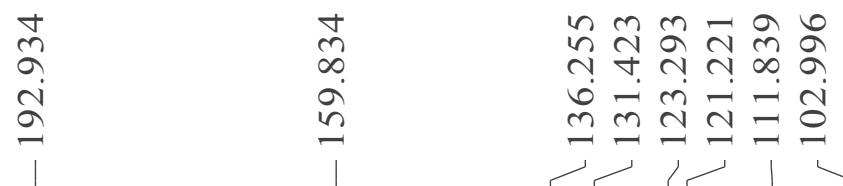

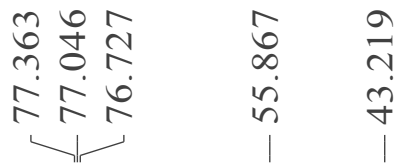

$\stackrel{8}{:}$<smiles>COc1ccccc1C(=O)C[Se]#N</smiles>

Chemical Shift (ppm) 
${ }^{1} \mathrm{H}$ NMR (400 MHz, $\mathrm{CDCl}_{3}$ ) spectrum of 2-selenocyanato-1-(o-tolyl)ethan-1-one (31)

\begin{tabular}{|c|c|c|}
\hline 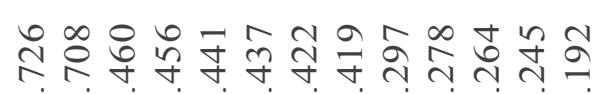 & $\vec{\infty}$ & $\begin{array}{l}\infty \\
\stackrel{a}{+}\end{array}$ \\
\hline 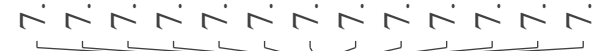 & $\dot{\sigma}$ & $\dot{\sim}$ \\
\hline
\end{tabular}

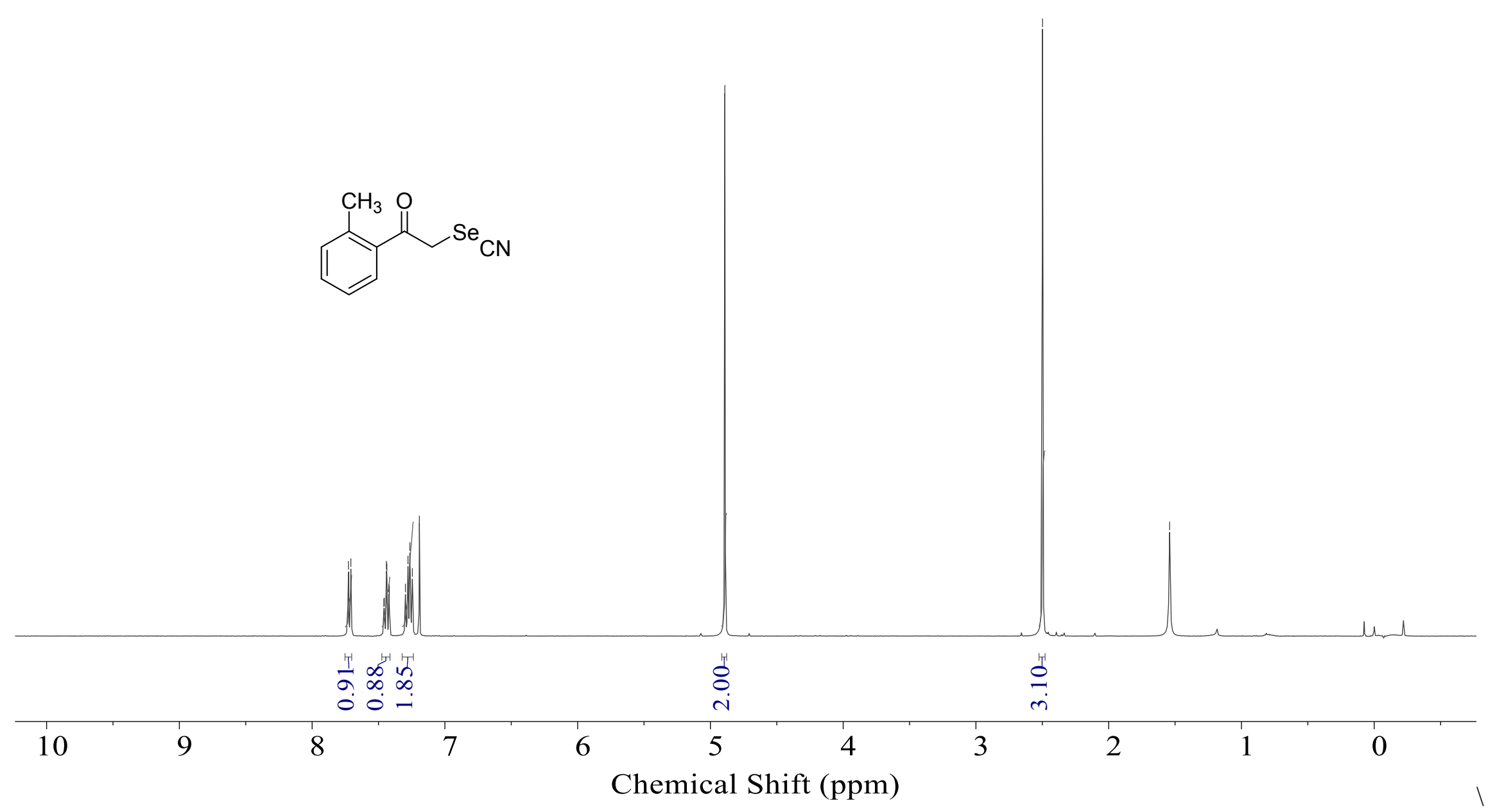


${ }^{13} \mathrm{C}\left\{{ }^{1} \mathrm{H}\right\}$ NMR $\left(100 \mathrm{MHz}, \mathrm{CDCl}_{3}\right)$ spectrum of 2-selenocyanato-1-(o-tolyl)ethan-1-one (3I)

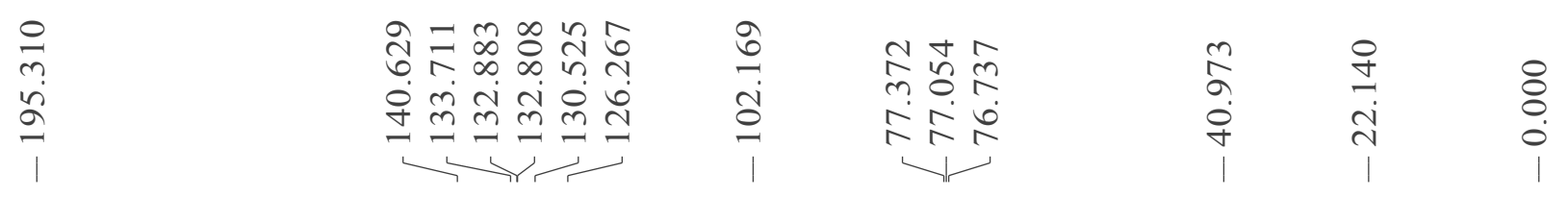
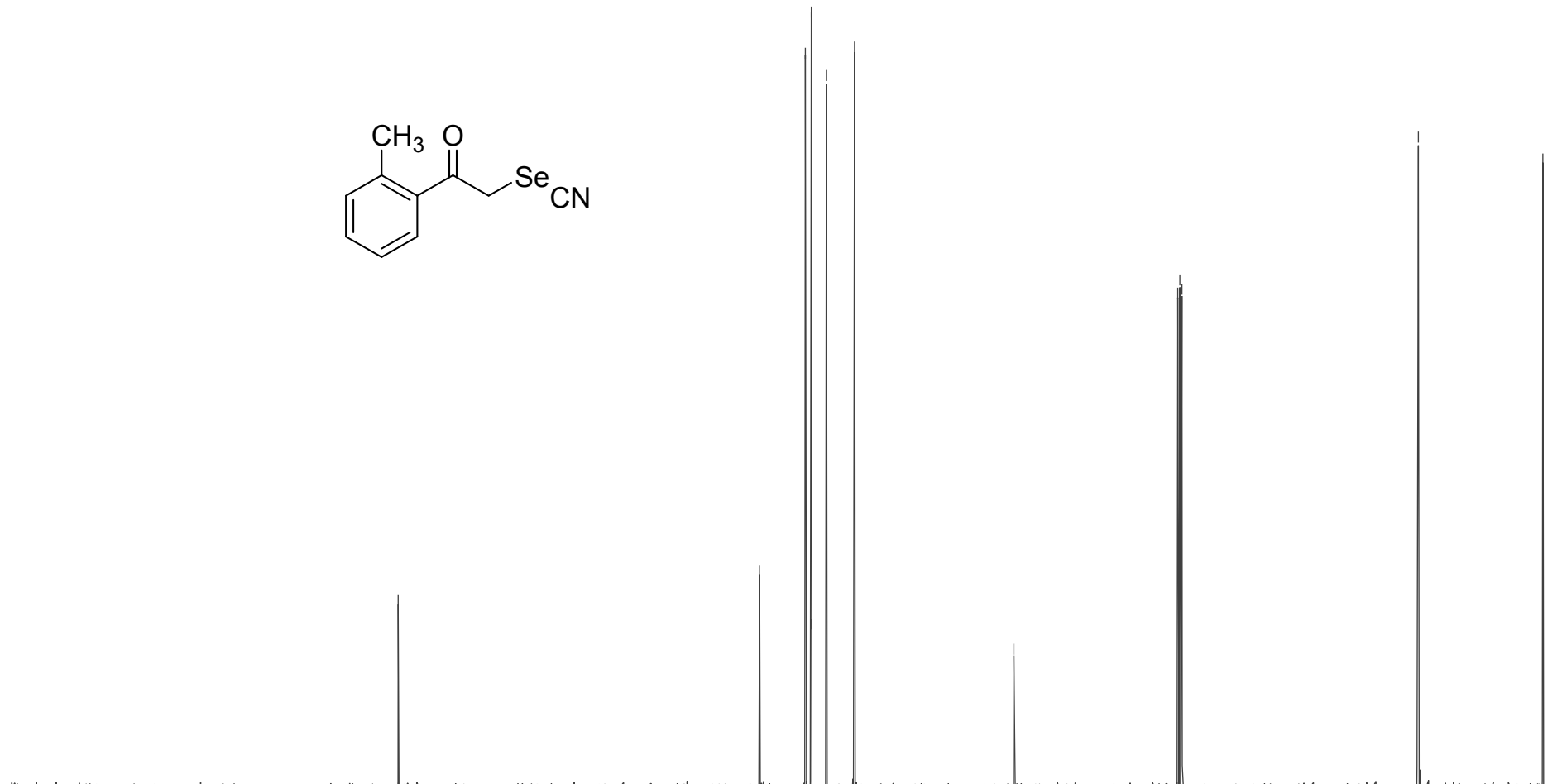

104

Chemical Shift (ppm) 
${ }^{1} \mathrm{H}$ NMR (400 MHz, $\mathrm{CDCl}_{3}$ ) spectrum of 1-(2-chlorophenyl)-2-selenocyanatoethan-1-one (3m)
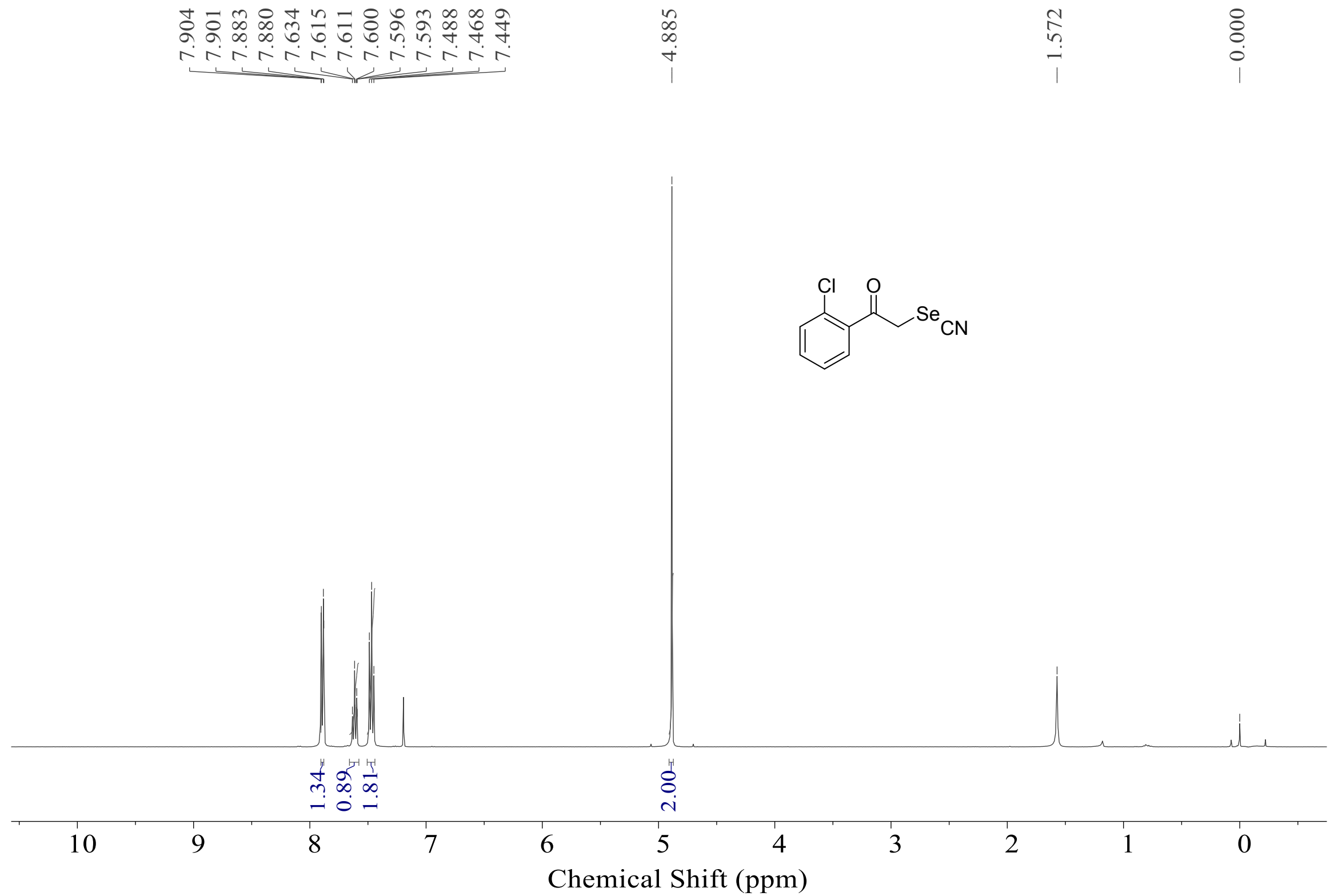
${ }^{13} \mathrm{C}\left\{{ }^{1} \mathrm{H}\right\}$ NMR (100 MHz, $\left.\mathrm{CDCl}_{3}\right)$ spectrum of 1-(2-chlorophenyl)-2-selenocyanatoethan-1-one (3m)

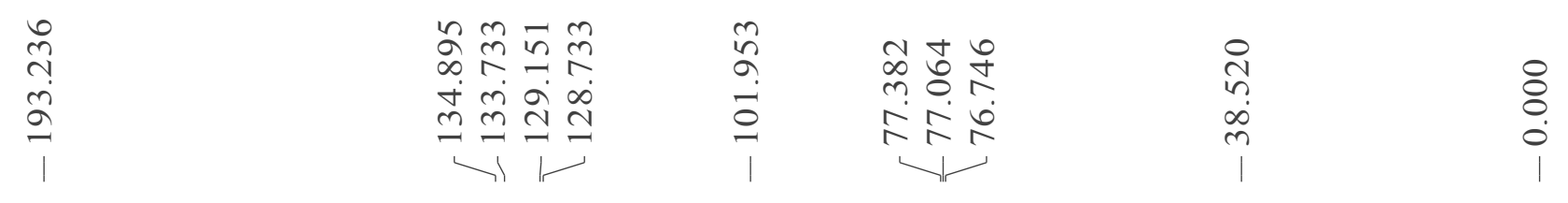

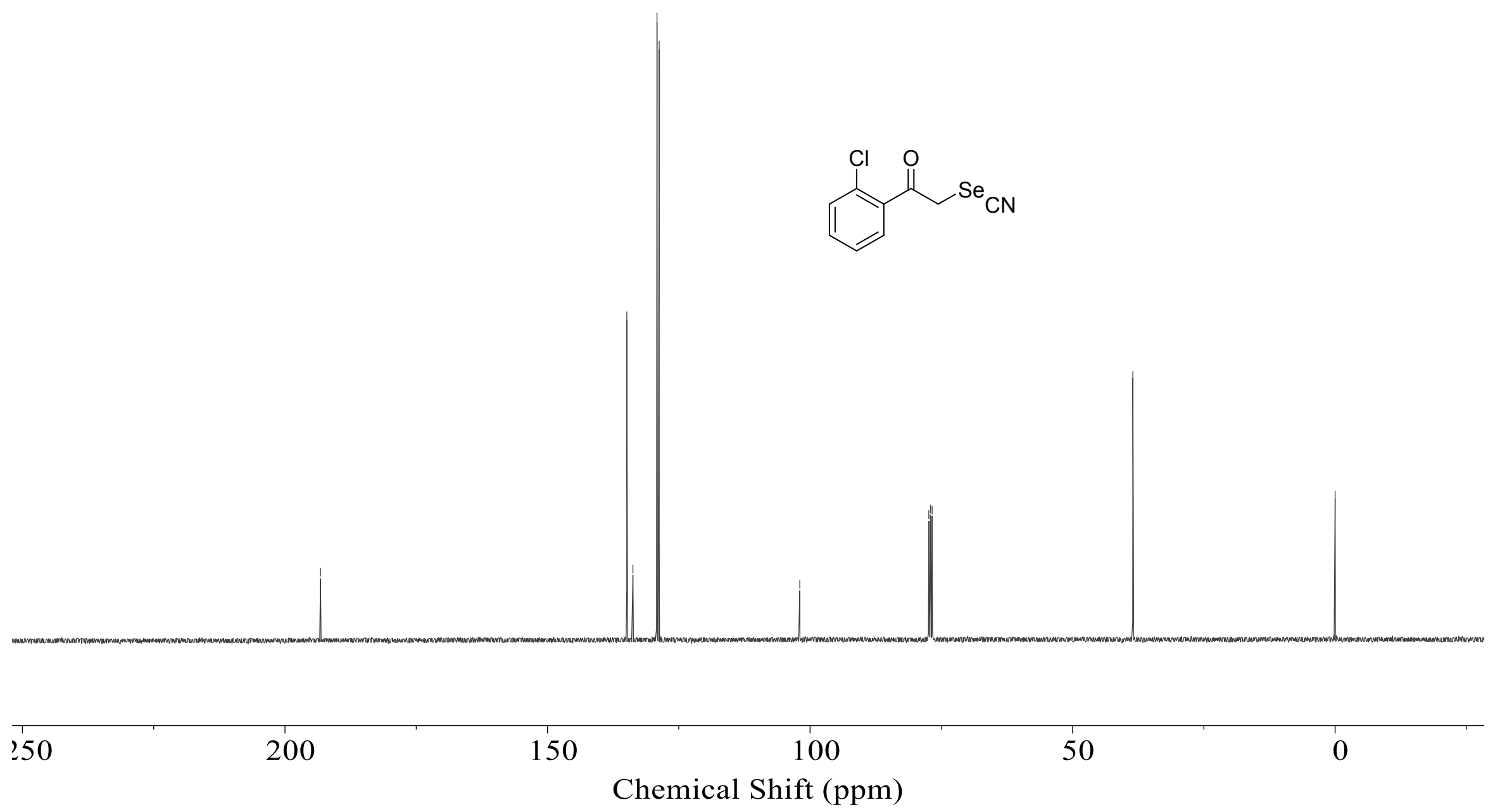


${ }^{1} \mathrm{H}$ NMR (400 MHz, $\mathrm{CDCl}_{3}$ ) spectrum of 1-(2-bromophenyl)-2-selenocyanatoethan-1-one (3n)
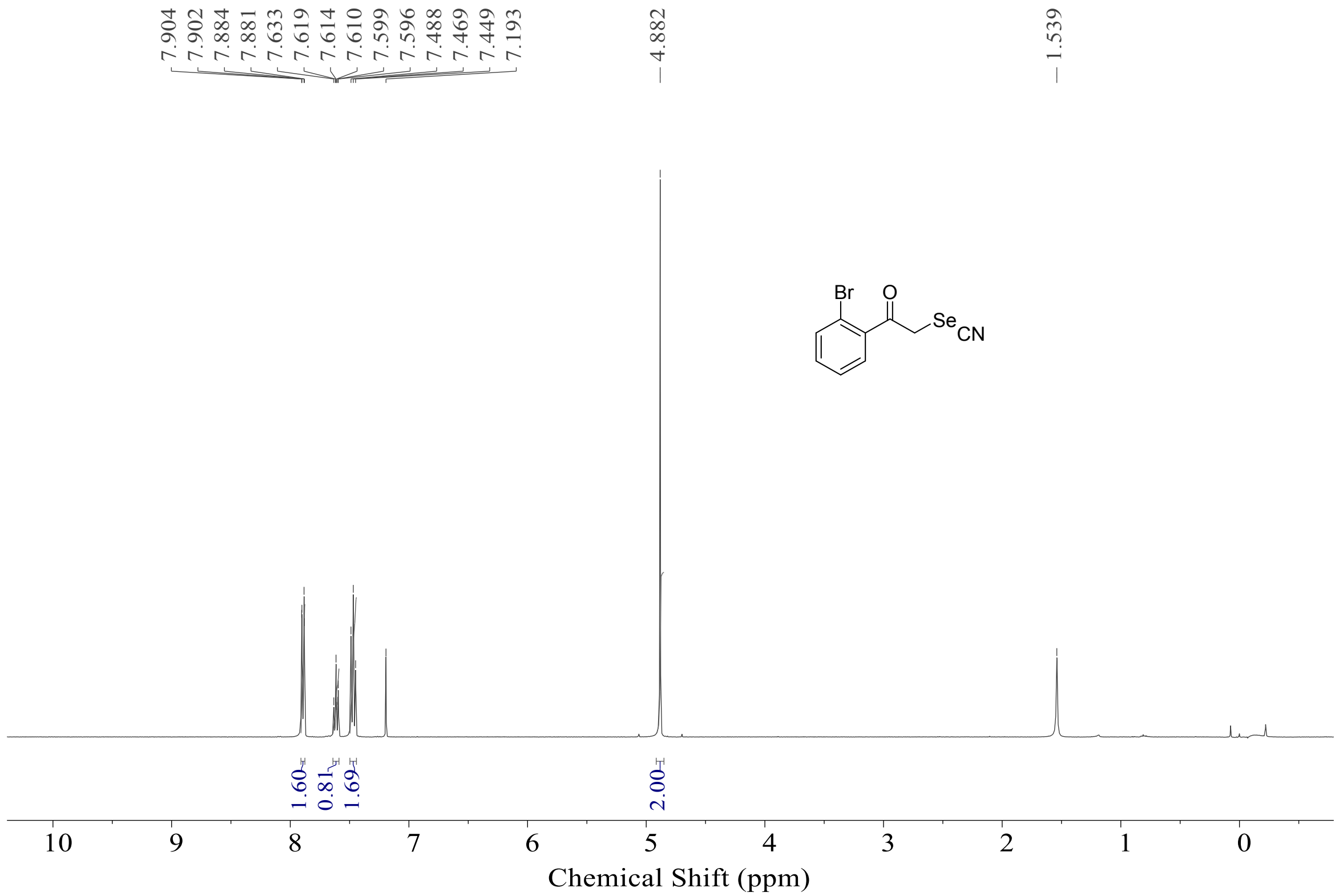
${ }^{13} \mathrm{C}\left\{{ }^{1} \mathrm{H}\right\}$ NMR (100 MHz, $\left.\mathrm{CDCl}_{3}\right)$ spectrum of 1-(2-bromophenyl)-2-selenocyanatoethan-1-one (3n)

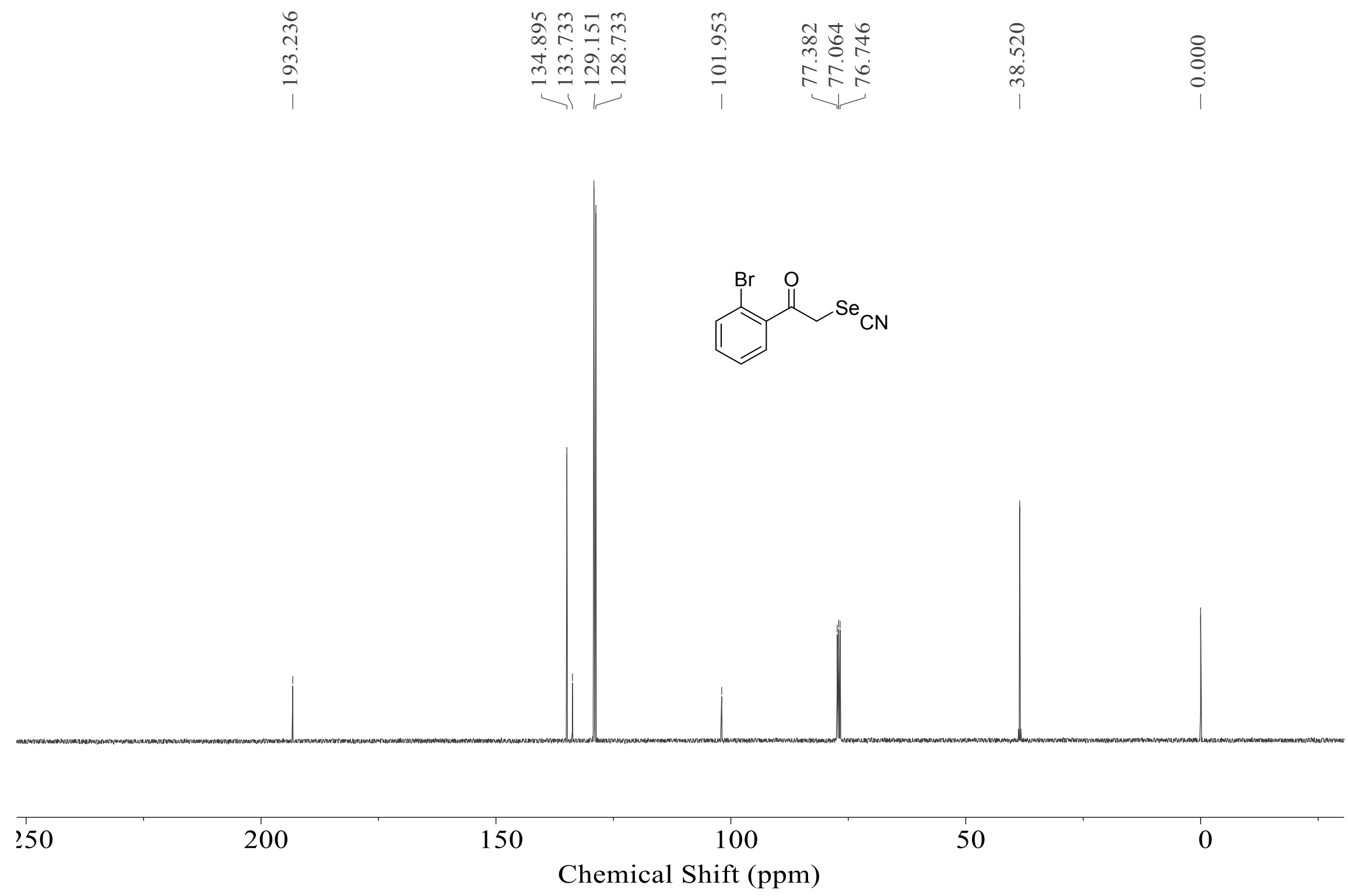


${ }^{1} \mathrm{H}$ NMR (400 MHz, $\mathrm{CDCl}_{3}$ ) spectrum of 1-(2,4-dimethoxyphenyl)-2-selenocyanatoethan-1-one (3o)

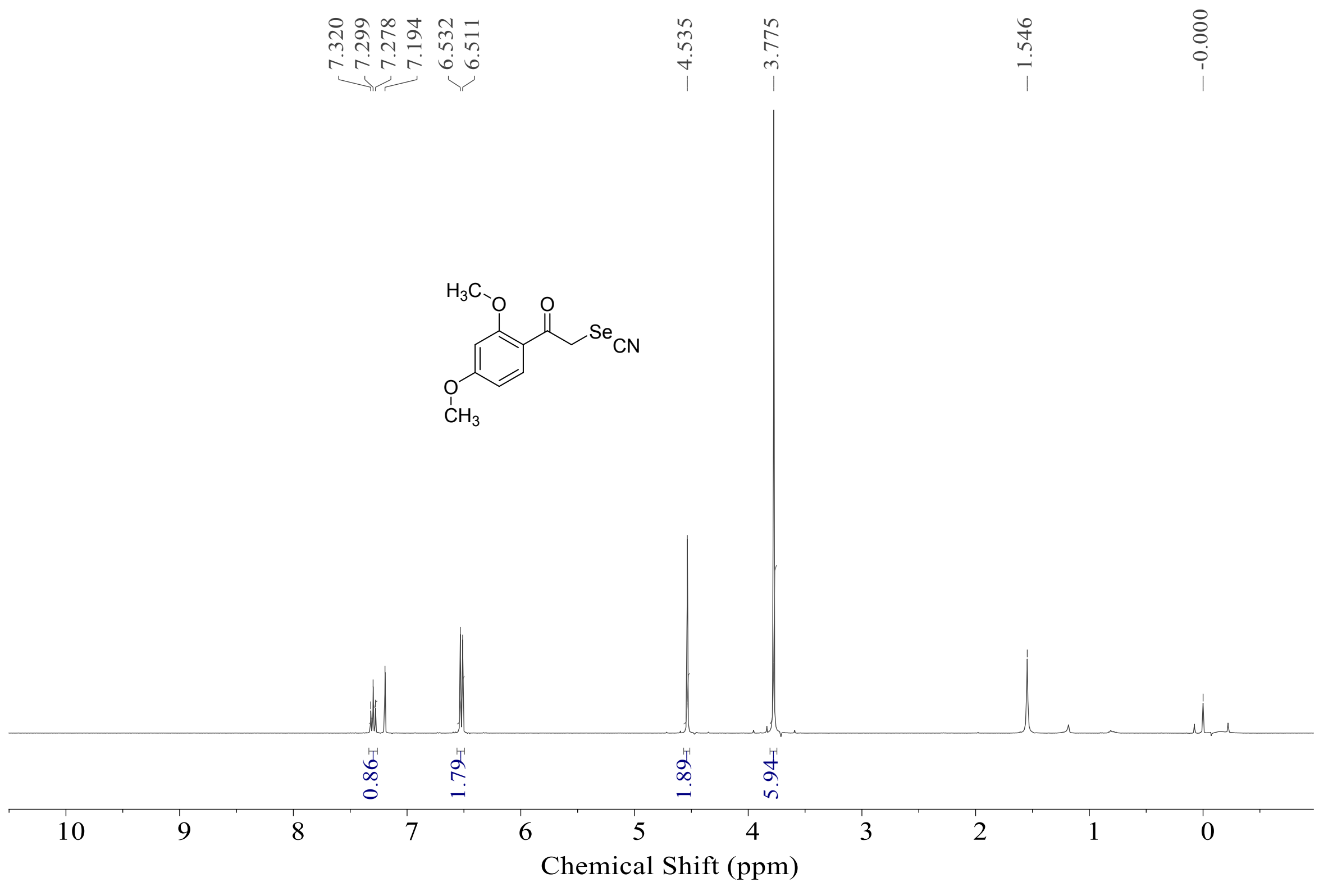


${ }^{13} \mathrm{C}\left\{{ }^{1} \mathrm{H}\right\}$ NMR $\left(100 \mathrm{MHz}, \mathrm{CDCl}_{3}\right)$ spectrum of 1-(2,4-dimethoxyphenyl)-2-selenocyanatoethan-1-one (3o)

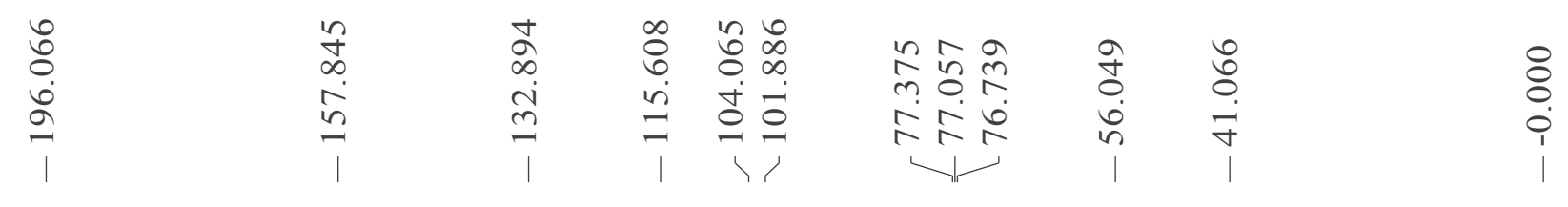

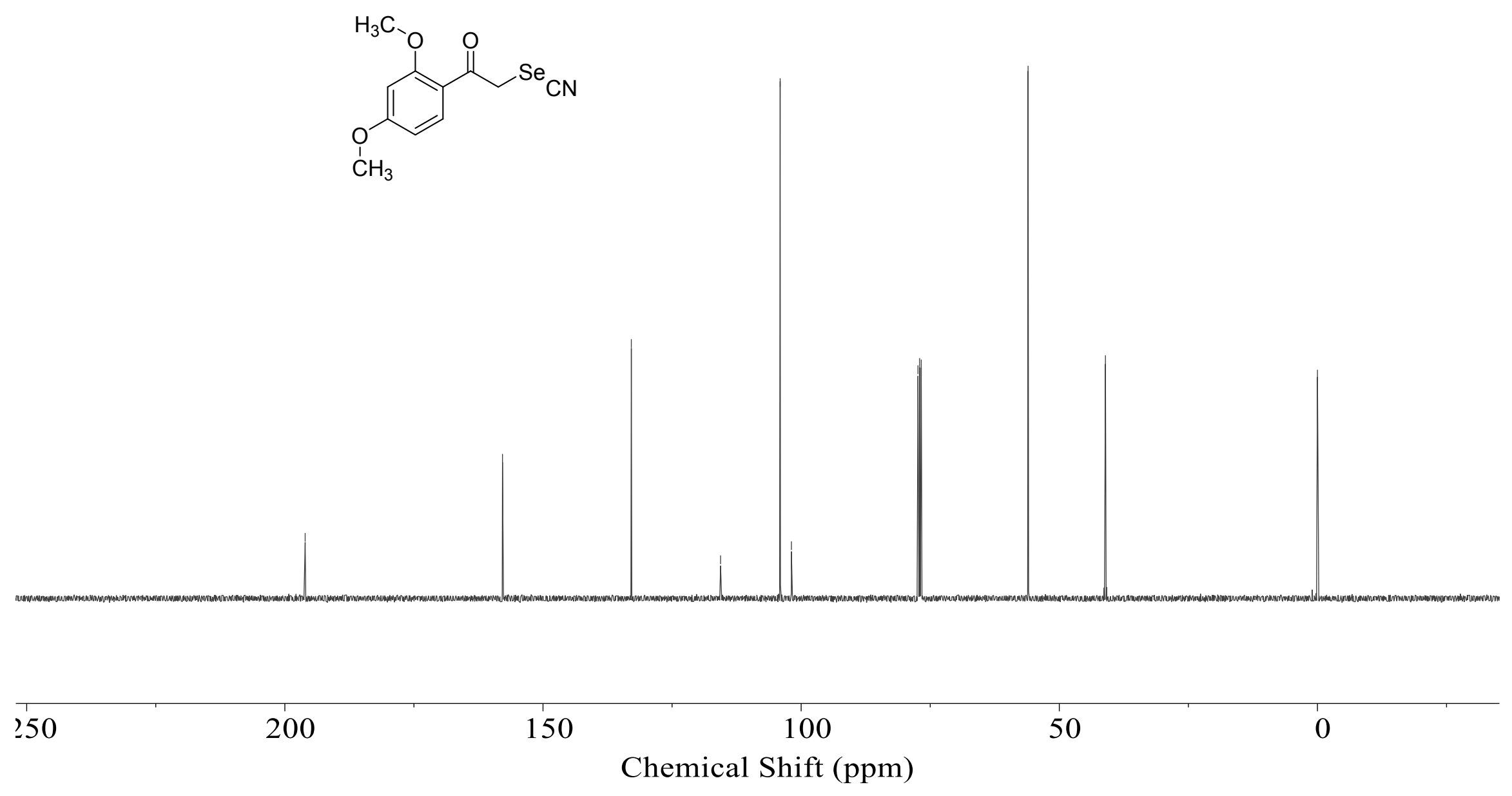


${ }^{1} \mathrm{H}$ NMR (400 MHz, $\mathrm{CDCl}_{3}$ ) spectrum of 1-(2,4-dimethylphenyl)-2-selenocyanatoethan-1-one (3p)

\begin{tabular}{|c|c|c|}
\hline 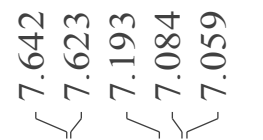 & $\begin{array}{l}8 \\
\& \\
\dot{+}\end{array}$ & 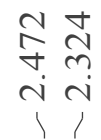 \\
\hline
\end{tabular}

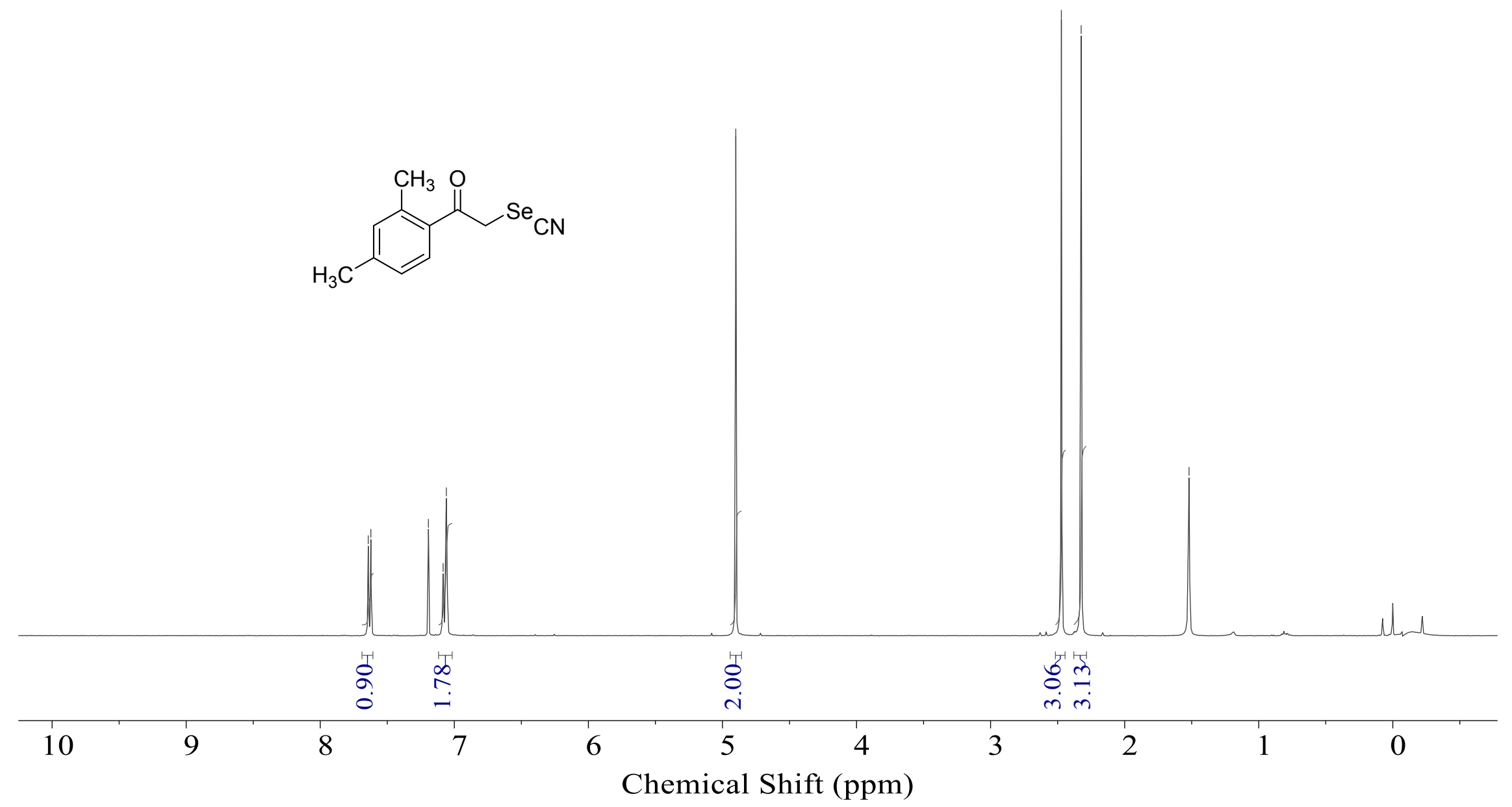


${ }^{13} \mathrm{C}\left\{{ }^{1} \mathrm{H}\right\}$ NMR (100 MHz, $\left.\mathrm{CDCl}_{3}\right)$ spectrum of 1-(2,4-dimethylphenyl)-2-selenocyanatoethan-1-one (3p)

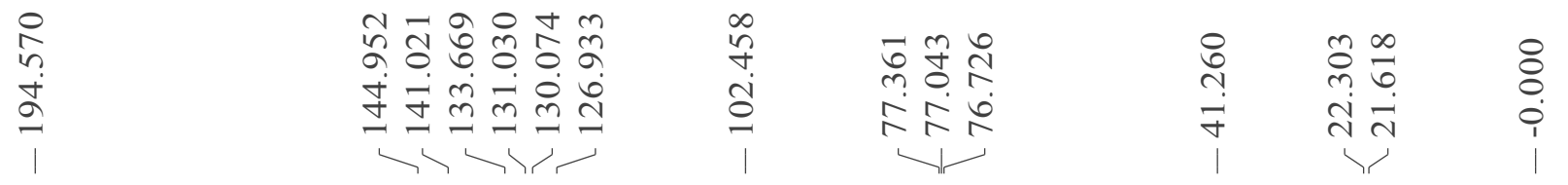<smiles>Cc1ccc(C(=O)C[Se])c(C)c1</smiles>

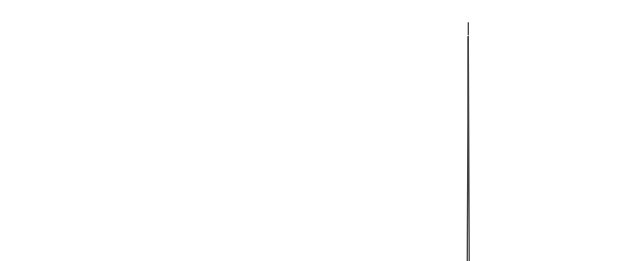

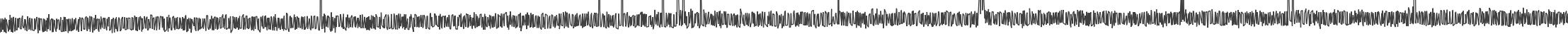

\section{Chemical Shift (ppm)}


${ }^{1} \mathrm{H} \mathrm{NMR}\left(400 \mathrm{MHz}, \mathrm{CDCl}_{3}\right.$ ) spectrum of 1-mesityl-2-selenocyanatoethan-1-one (3q)

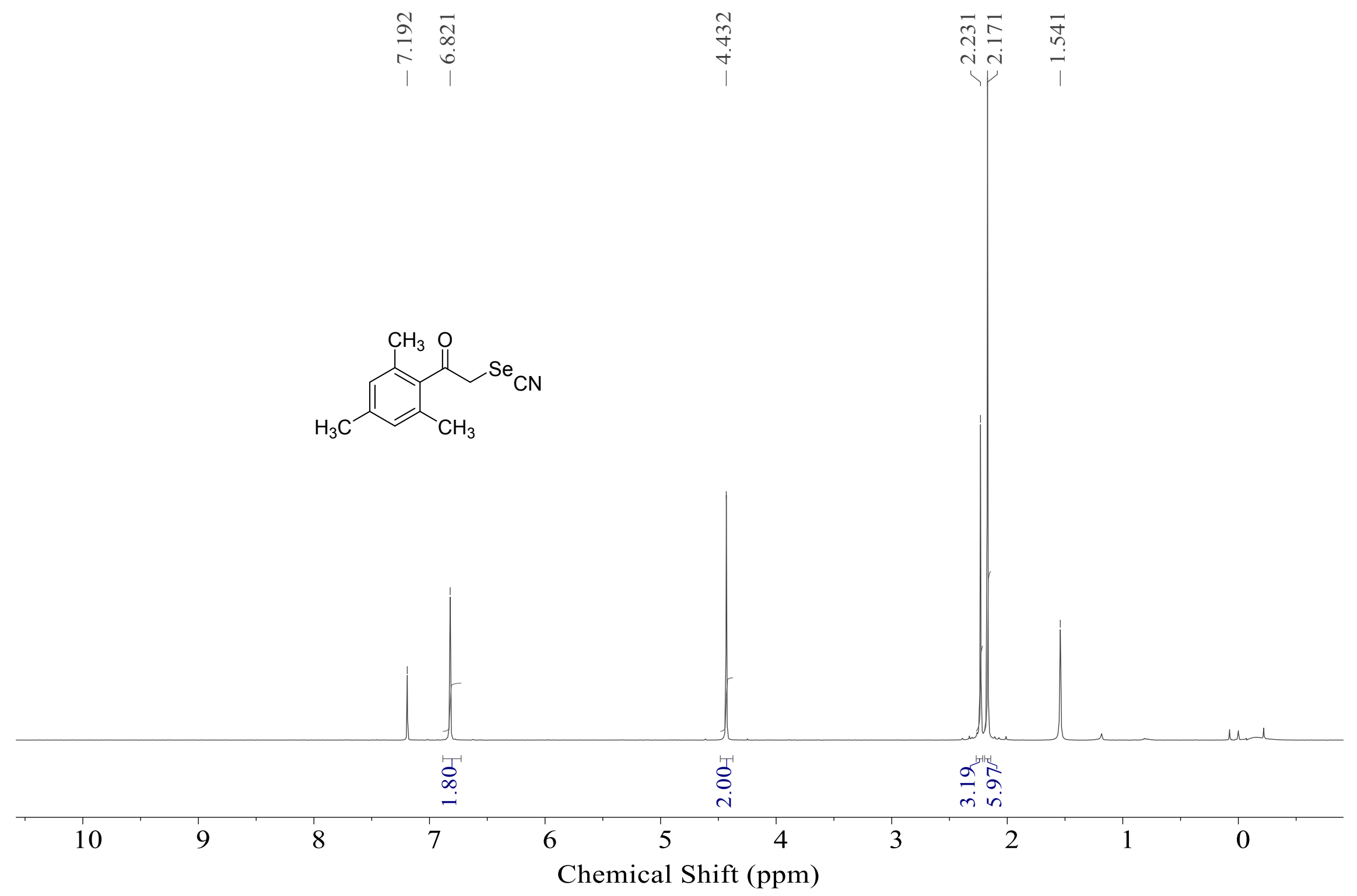


${ }^{13} \mathrm{C}\left\{{ }^{1} \mathrm{H}\right\}$ NMR (100 MHz, $\left.\mathrm{CDCl}_{3}\right)$ spectrum of 1-mesityl-2-selenocyanatoethan-1-one (3q)

\begin{tabular}{|c|c|c|c|c|c|}
\hline $\begin{array}{l}\text { ơ } \\
\text { ô } \\
\text { ì } \\
\text { ते }\end{array}$ & 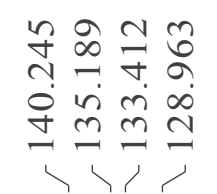 & 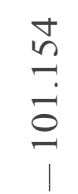 & 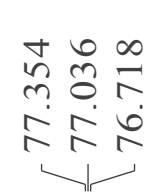 & $\begin{array}{c}\hat{ָ} \\
\dot{\sigma} \\
\dot{\sigma}\end{array}$ & 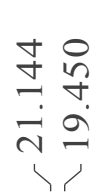 \\
\hline
\end{tabular}

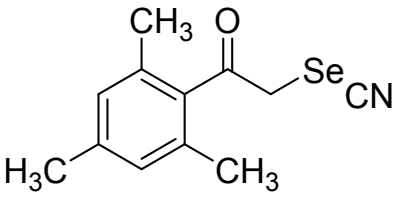

\section{Chemical Shift (ppm)}


${ }^{1} \mathrm{H}$ NMR (400 MHz, $\mathrm{CDCl}_{3}$ ) spectrum of 1-(furan-2-yl)-2-selenocyanatoethan-1-one (3r)

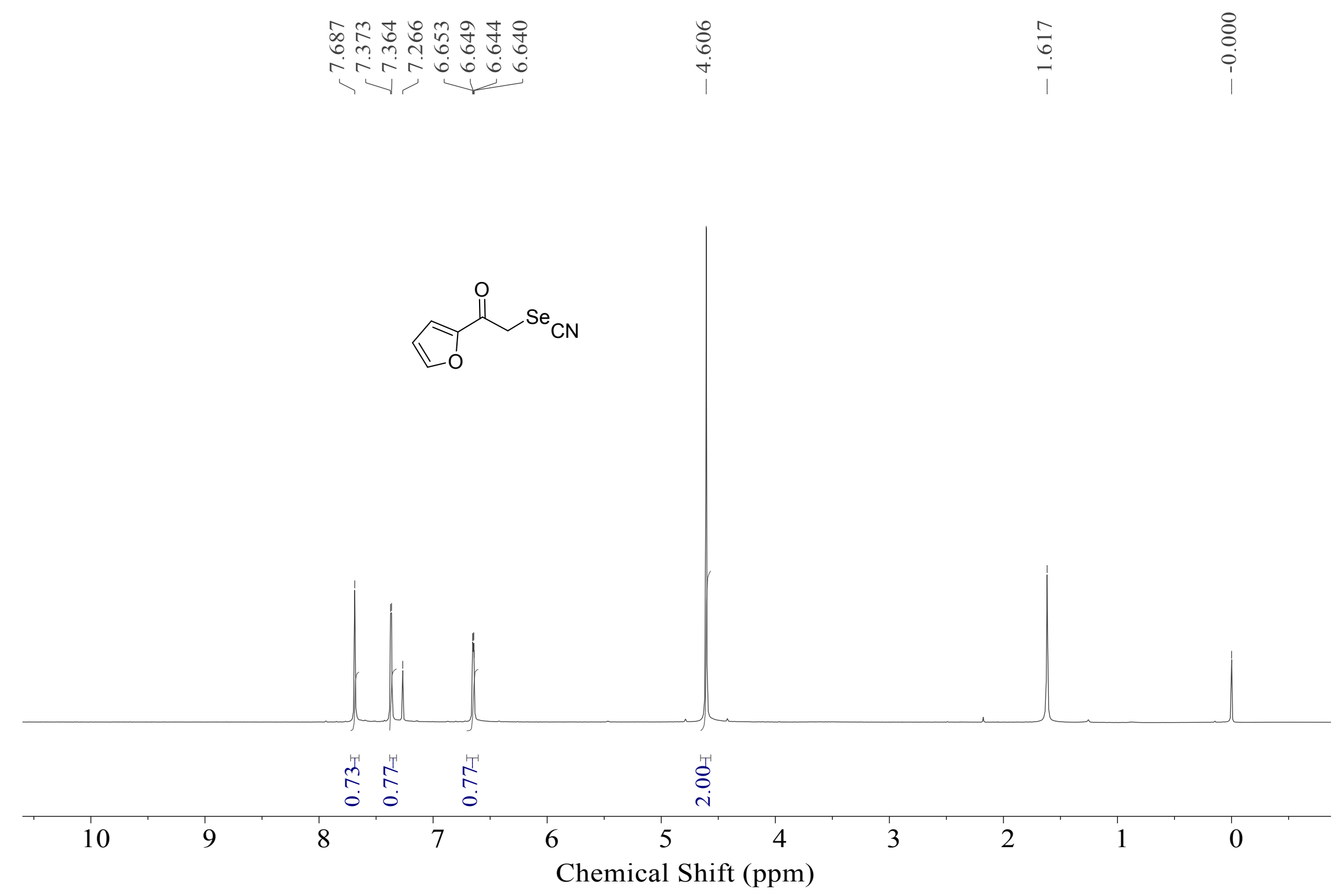


${ }^{13} \mathrm{C}\left\{{ }^{1} \mathrm{H}\right\}$ NMR (100 MHz, $\left.\mathrm{CDCl}_{3}\right)$ spectrum of 1-(furan-2-yl)-2-selenocyanatoethan-1-one (3r)

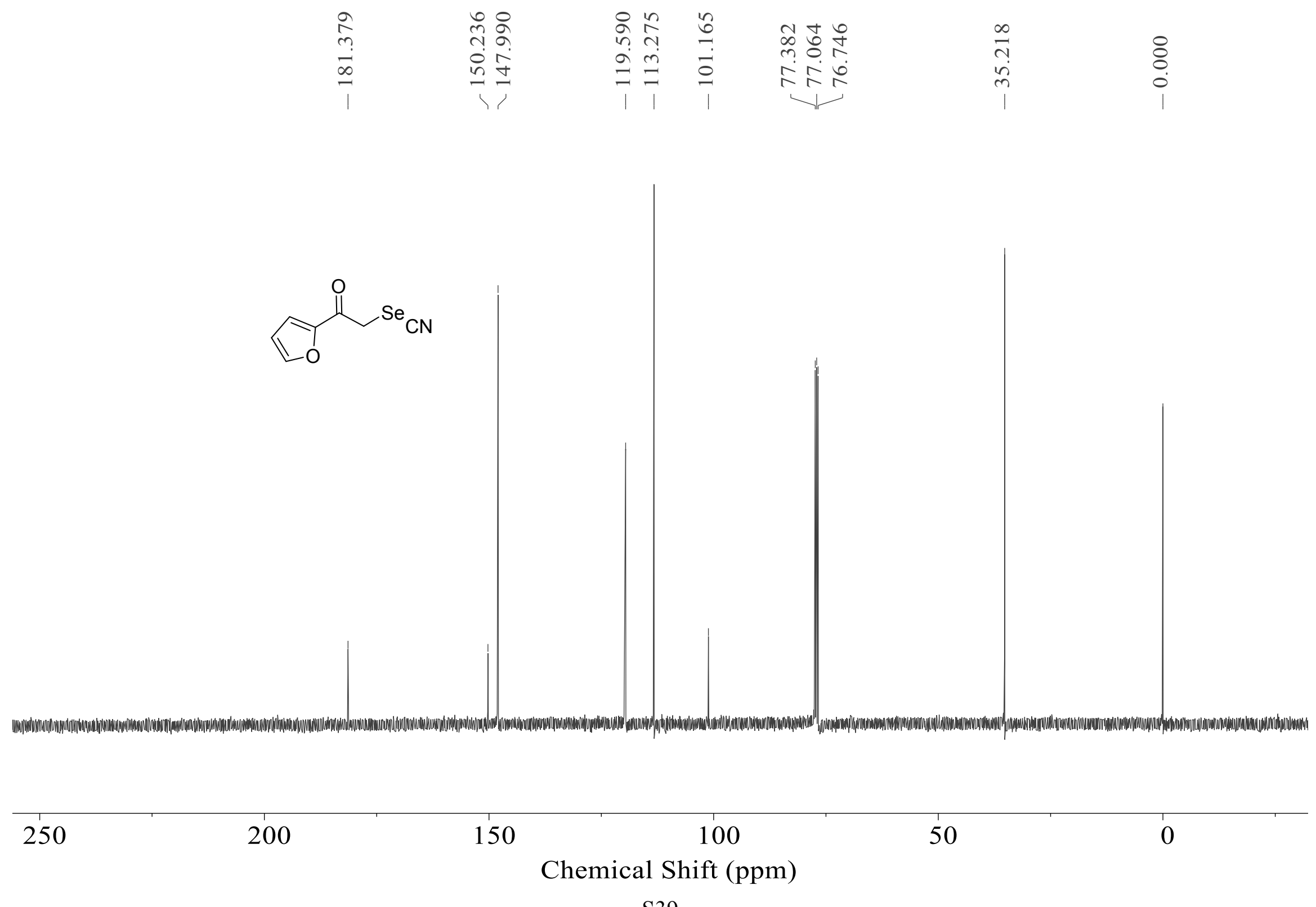


${ }^{1} \mathrm{H}$ NMR (400 MHz, $\mathrm{CDCl}_{3}$ ) spectrum of 2-selenocyanato-1-(thiophen-2-yl)ethan-1-one (3s)
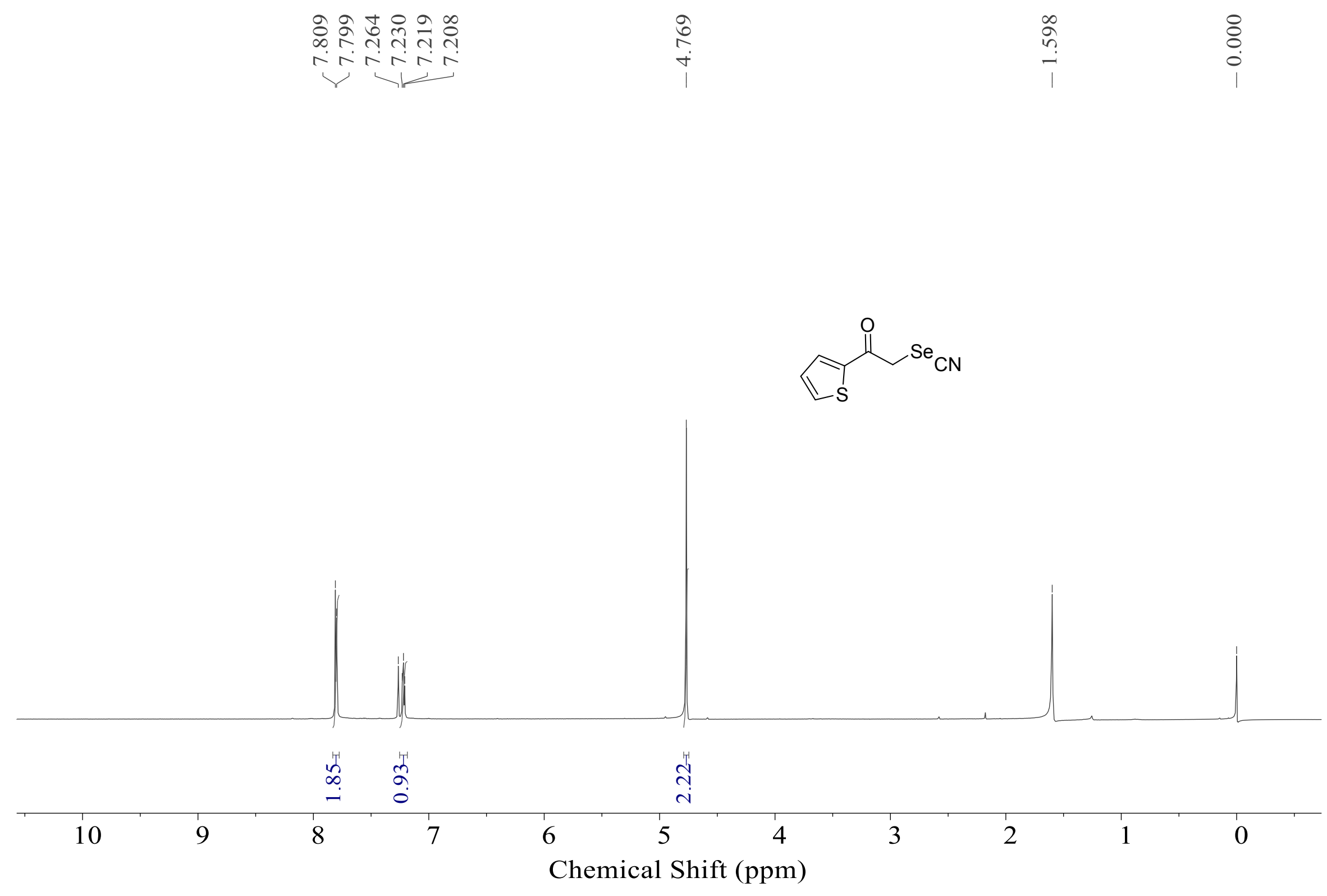
${ }^{13} \mathrm{C}\left\{{ }^{1} \mathrm{H}\right\}$ NMR $\left(100 \mathrm{MHz}, \mathrm{CDCl}_{3}\right)$ spectrum of 2-selenocyanato-1-(thiophen-2-yl)ethan-1-one (3s)

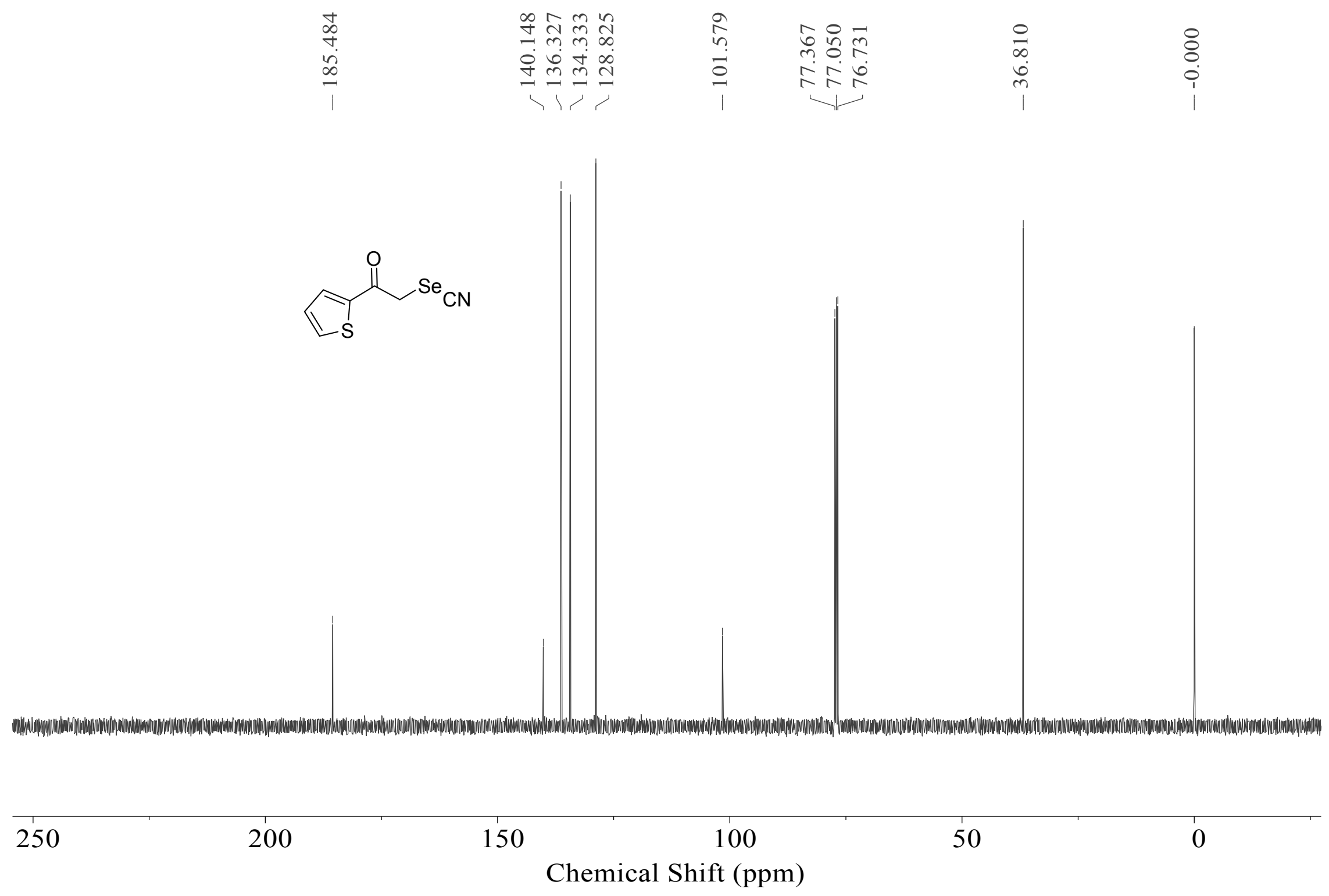


${ }^{1} \mathrm{H}$ NMR (400 MHz, $\mathrm{CDCl}_{3}$ ) spectrum of 4-phenyl-1-selenocyanatobut-3-en-2-one (5a)

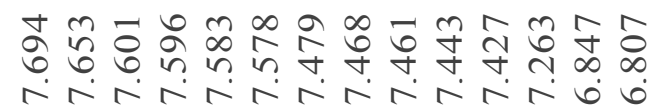

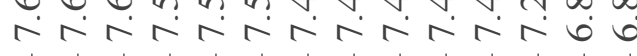

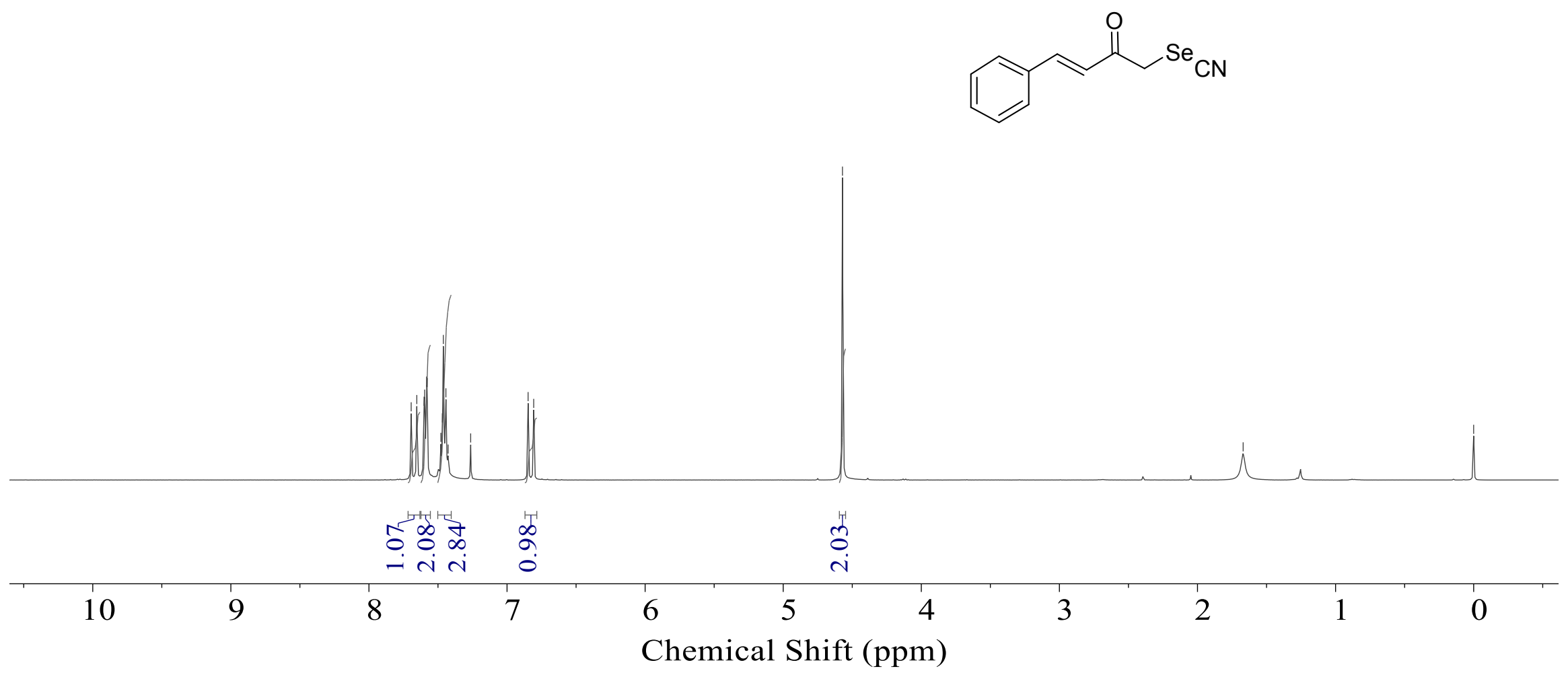


${ }^{13} \mathrm{C}\left\{{ }^{1} \mathrm{H}\right\}$ NMR (100 MHz, $\mathrm{CDCl}_{3}$ : DMSO-d ${ }_{6}$ ) spectrum of 4-phenyl-1-selenocyanatobut-3-en-2-one (5a)

\begin{tabular}{|c|c|c|c|c|c|}
\hline & $t$ & 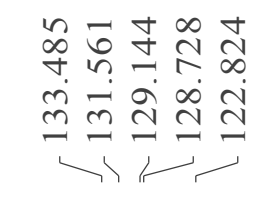 & 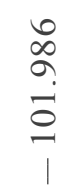 & 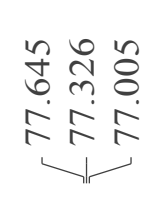 & 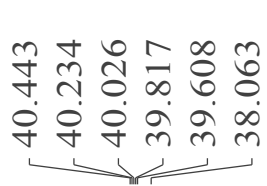 \\
\hline
\end{tabular}

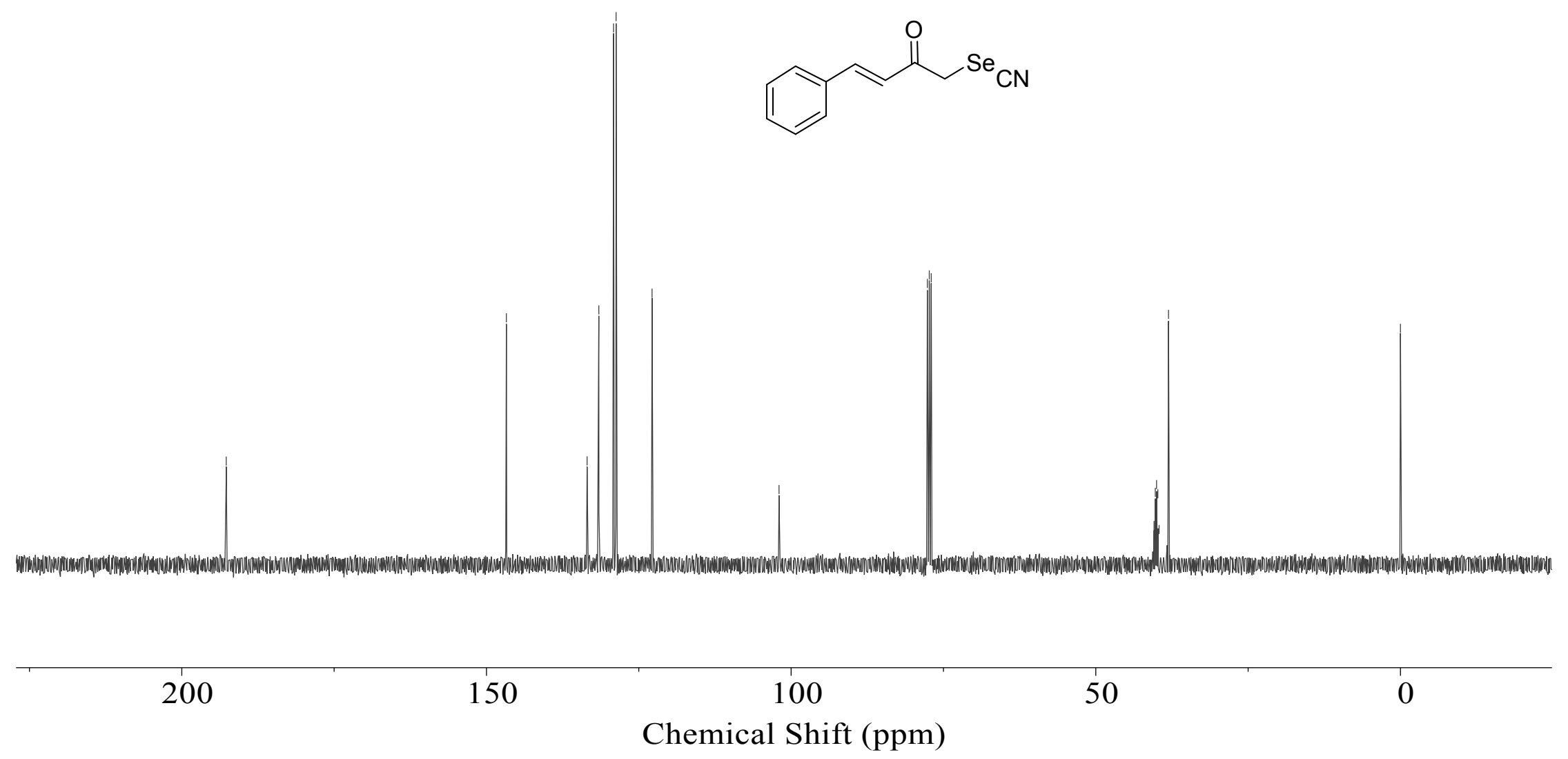


${ }^{1} \mathrm{H}$ NMR (400 MHz, $\mathrm{CDCl}_{3}$ ) spectrum of 1-selenocyanato-4-(p-tolyl)but-3-en-2-one (5b)
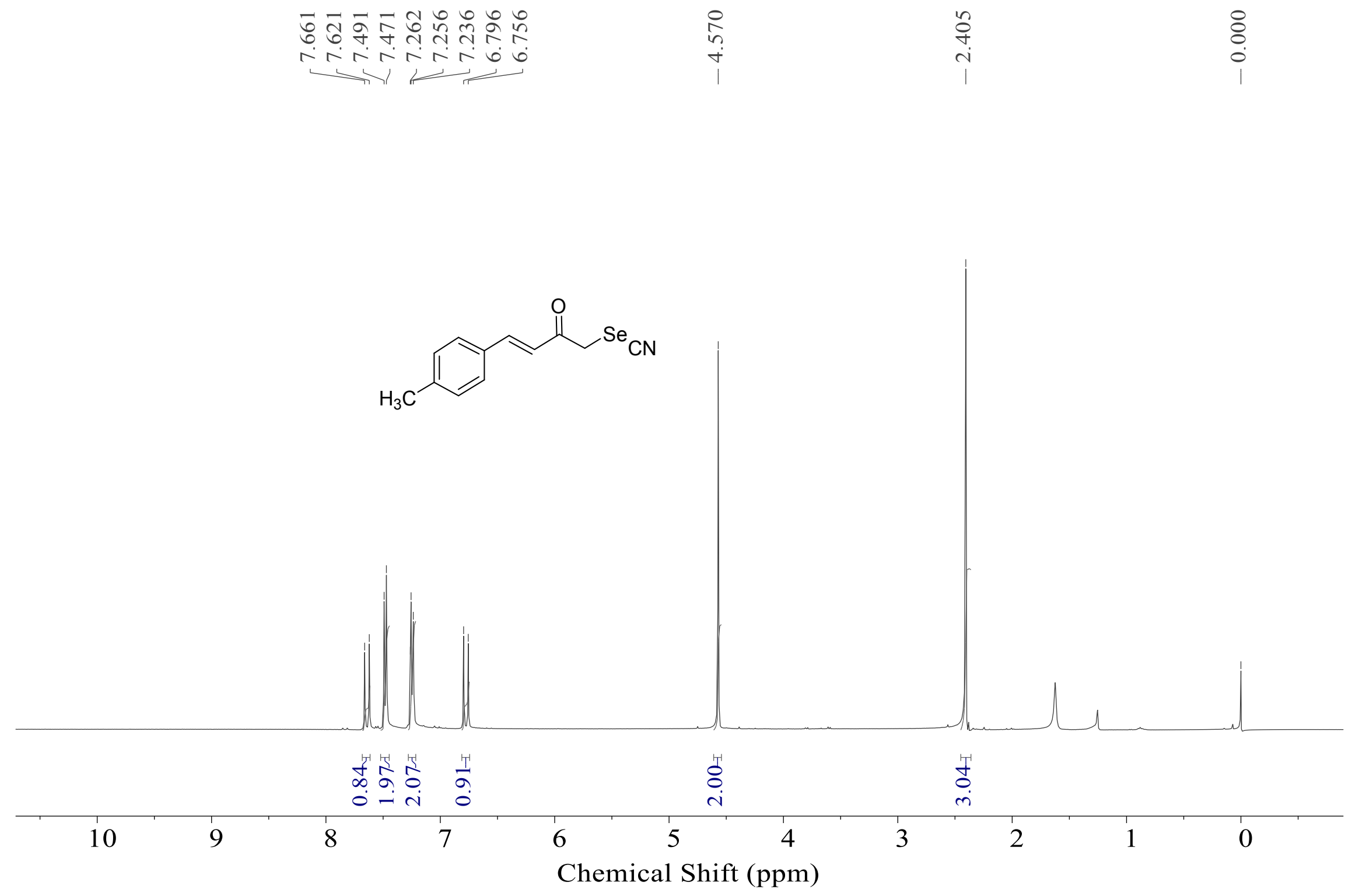
${ }^{13} \mathrm{C}\left\{{ }^{1} \mathrm{H}\right\}$ NMR (100 MHz, $\left.\mathrm{CDCl}_{3}\right)$ spectrum of 1-selenocyanato-4-(p-tolyl)but-3-en-2-one (5b)

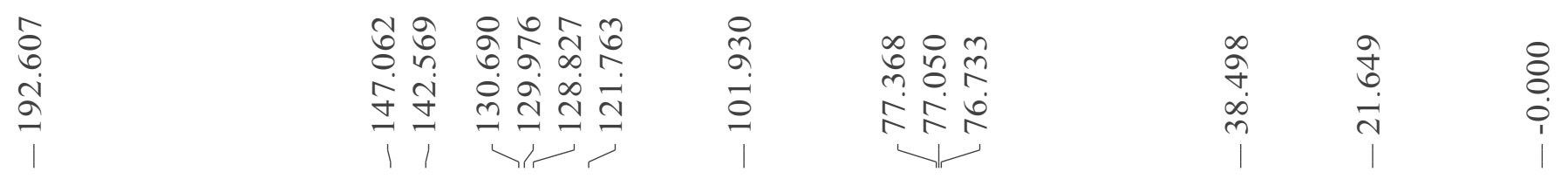

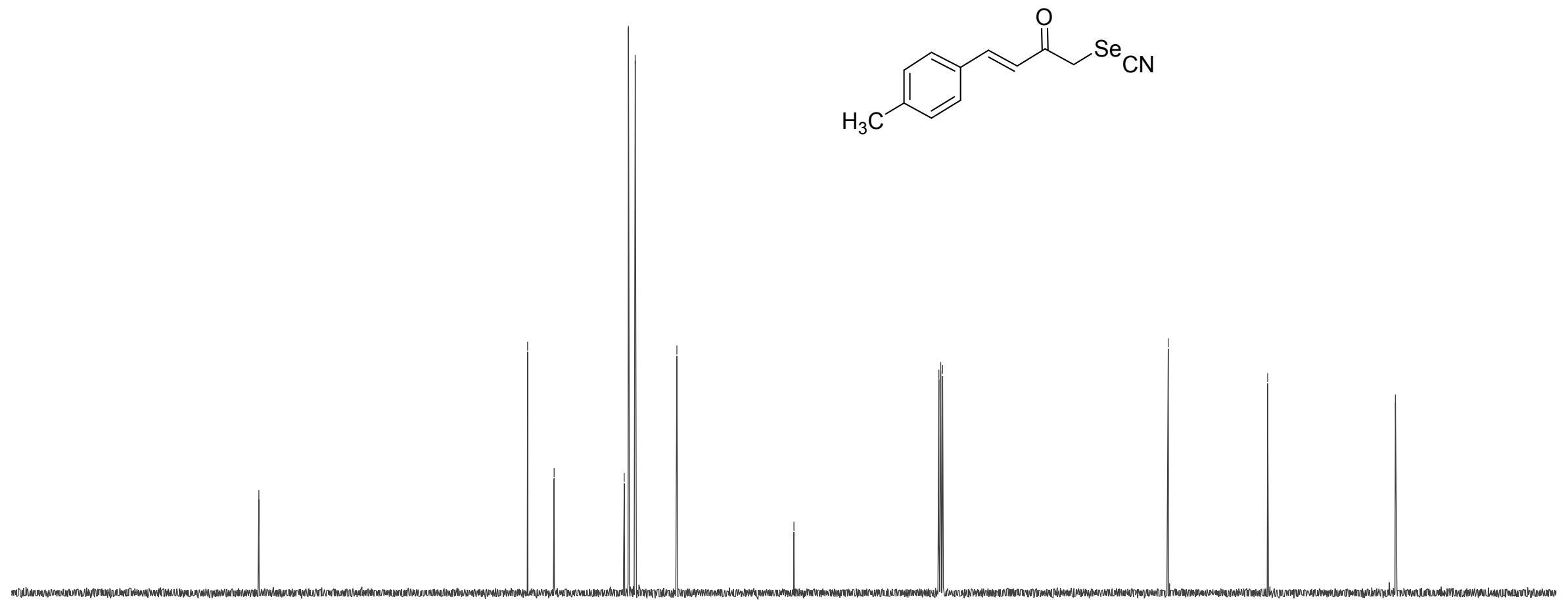

200

150

100

50

0

Chemical Shift (ppm) 
${ }^{1} \mathrm{H}$ NMR (400 MHz, $\mathrm{CDCl}_{3}$ ) spectrum of 4-(4-methoxyphenyl)-1-selenocyanatobut-3-en-2-one (5c)

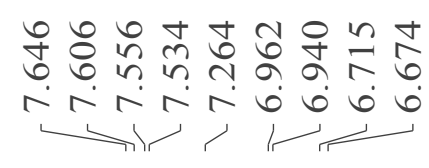

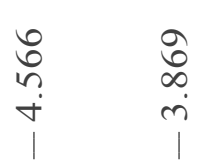

$\stackrel{0}{\frac{0}{6}}$

$\stackrel{8}{8}$

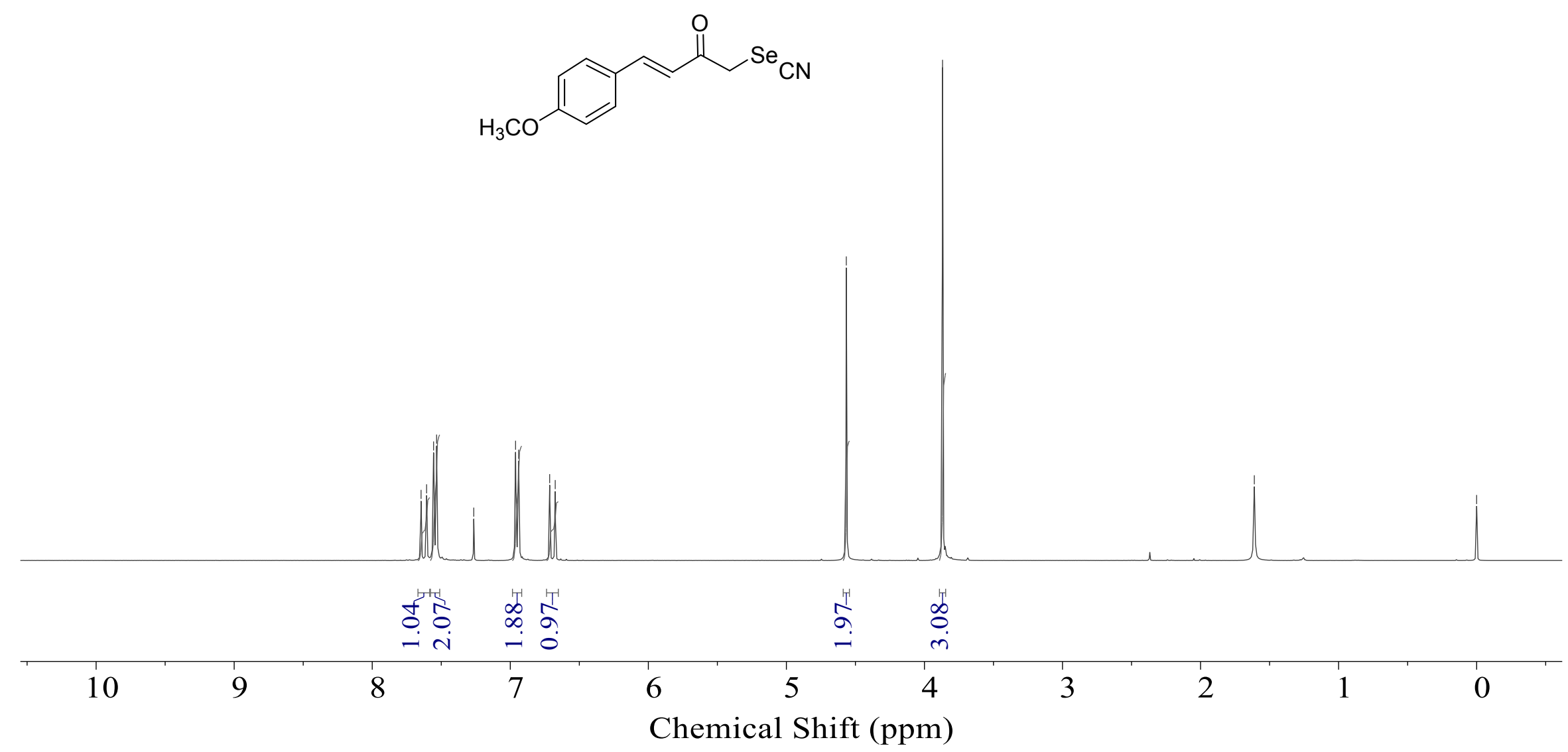


${ }^{13} \mathrm{C}\left\{{ }^{1} \mathrm{H}\right\}$ NMR (100 MHz, $\left.\mathrm{CDCl}_{3}\right)$ spectrum of 4-(4-methoxyphenyl)-1-selenocyanatobut-3-en-2-one (5c)

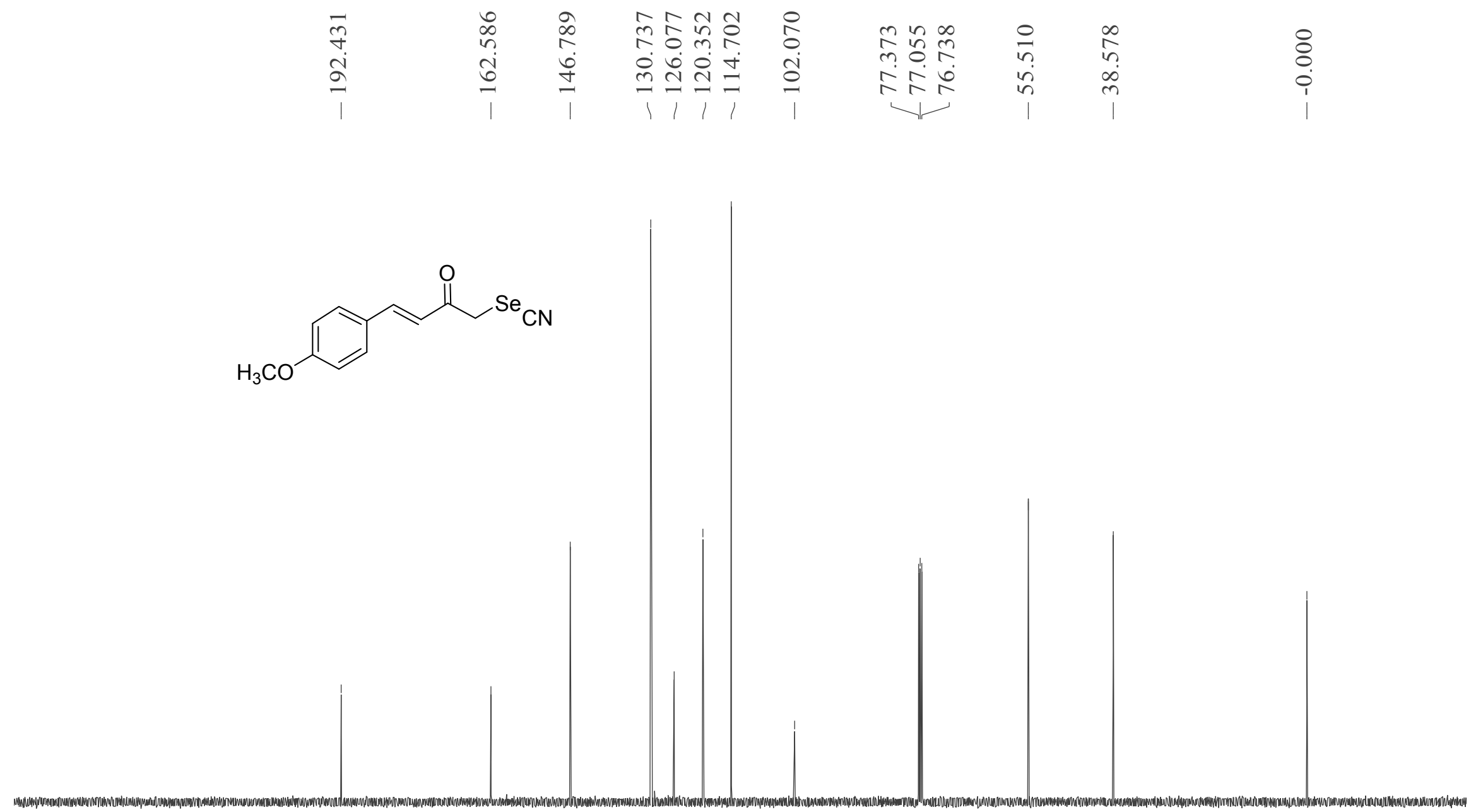

\section{0}

200

150

Chemical Shift (ppm)

50

0 
${ }^{1} \mathrm{H}$ NMR (400 MHz, $\mathrm{CDCl}_{3}$ ) spectrum of 4-(4-fluorophenyl)-1-selenocyanatobut-3-en-2-one (5d)

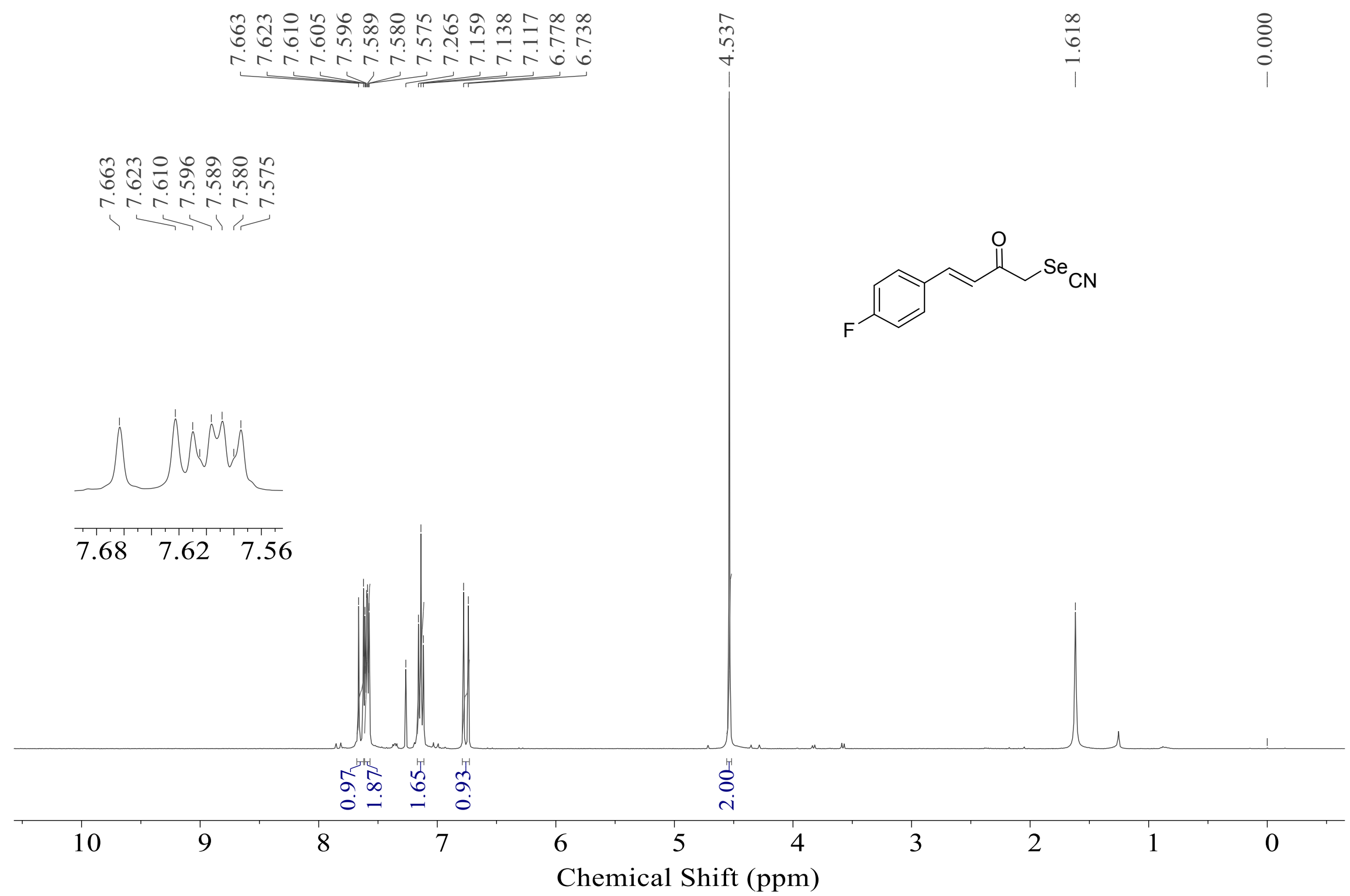


${ }^{13} \mathrm{C}\left\{{ }^{1} \mathrm{H}\right\}$ NMR (100 MHz, $\left.\mathrm{CDCl}_{3}\right)$ of spectrum 4-(4-fluorophenyl)-1-selenocyanatobut-3-en-2-one (5d)

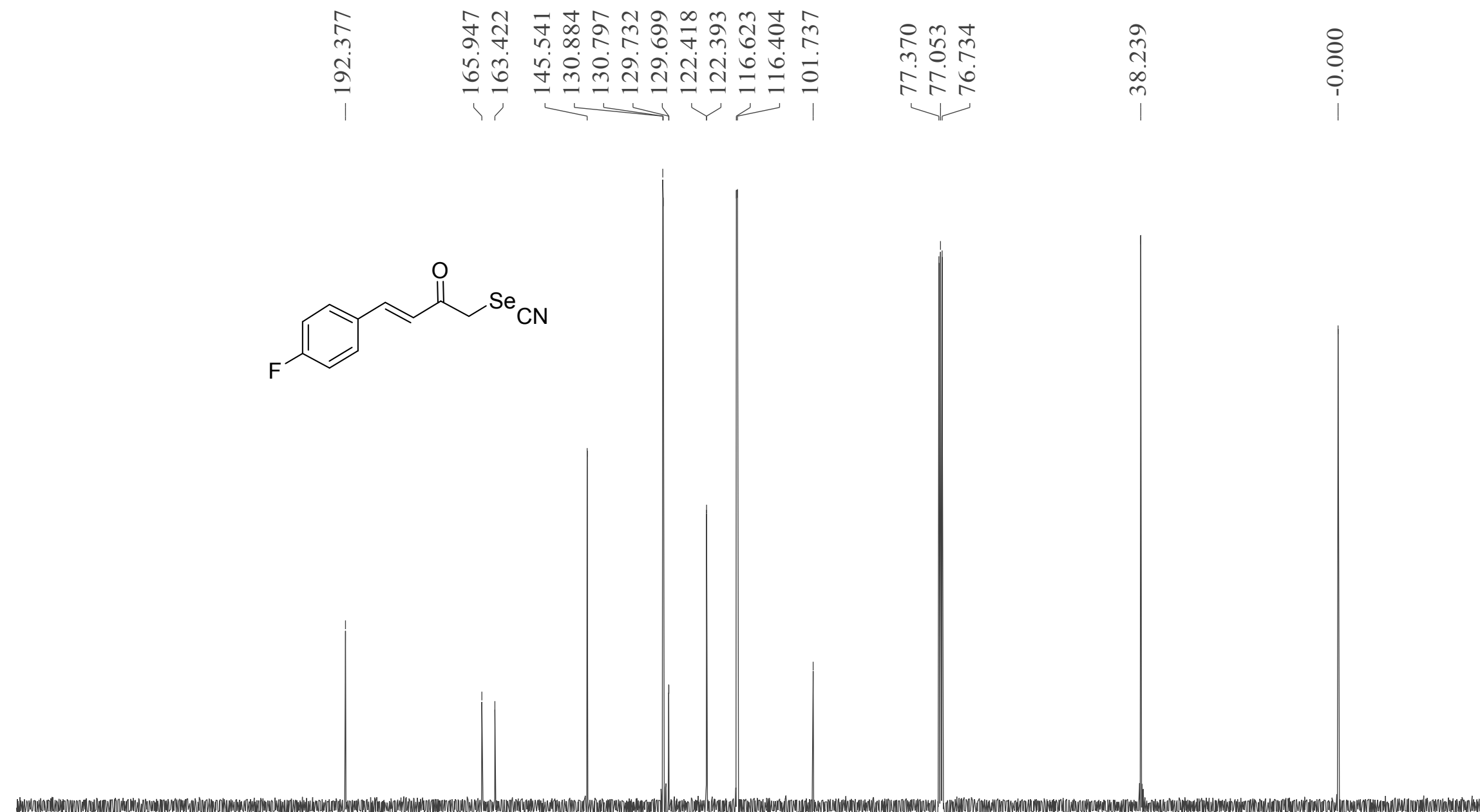

Chemical Shift (ppm) 
${ }^{1} \mathrm{H}$ NMR (400 MHz, $\mathrm{CDCl}_{3}$ ) spectrum of 4-(4-bromophenyl)-1-selenocyanatobut-3-en-2-one (5e)
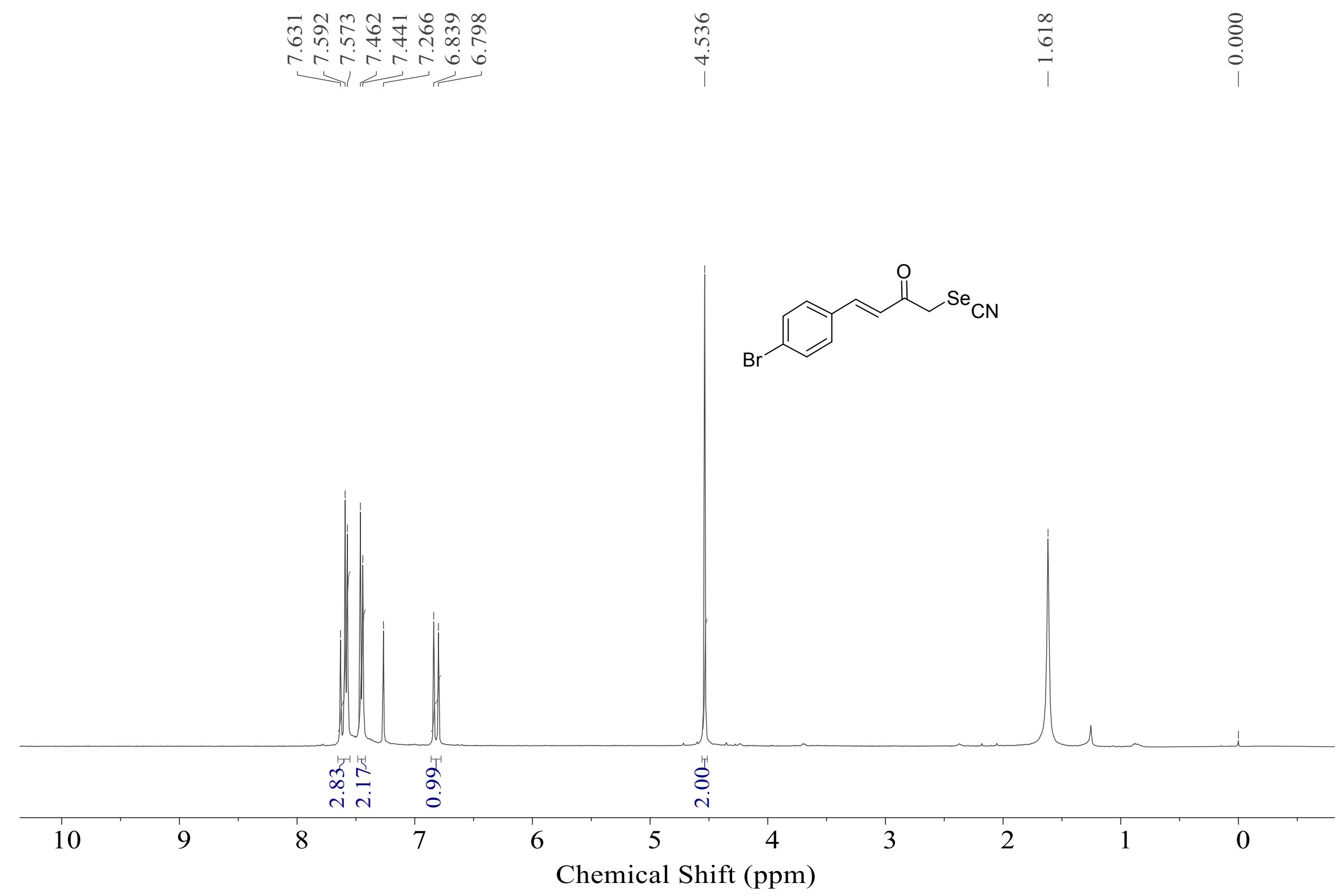
${ }^{13} \mathrm{C}\left\{{ }^{1} \mathrm{H}\right\}$ NMR (100 MHz, $\left.\mathrm{CDCl}_{3}\right)$ spectrum of 4-(4-bromophenyl)-1-selenocyanatobut-3-en-2-one (5e)

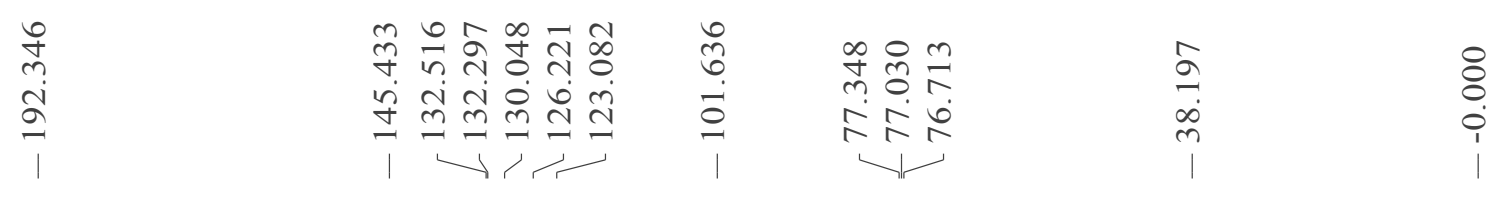

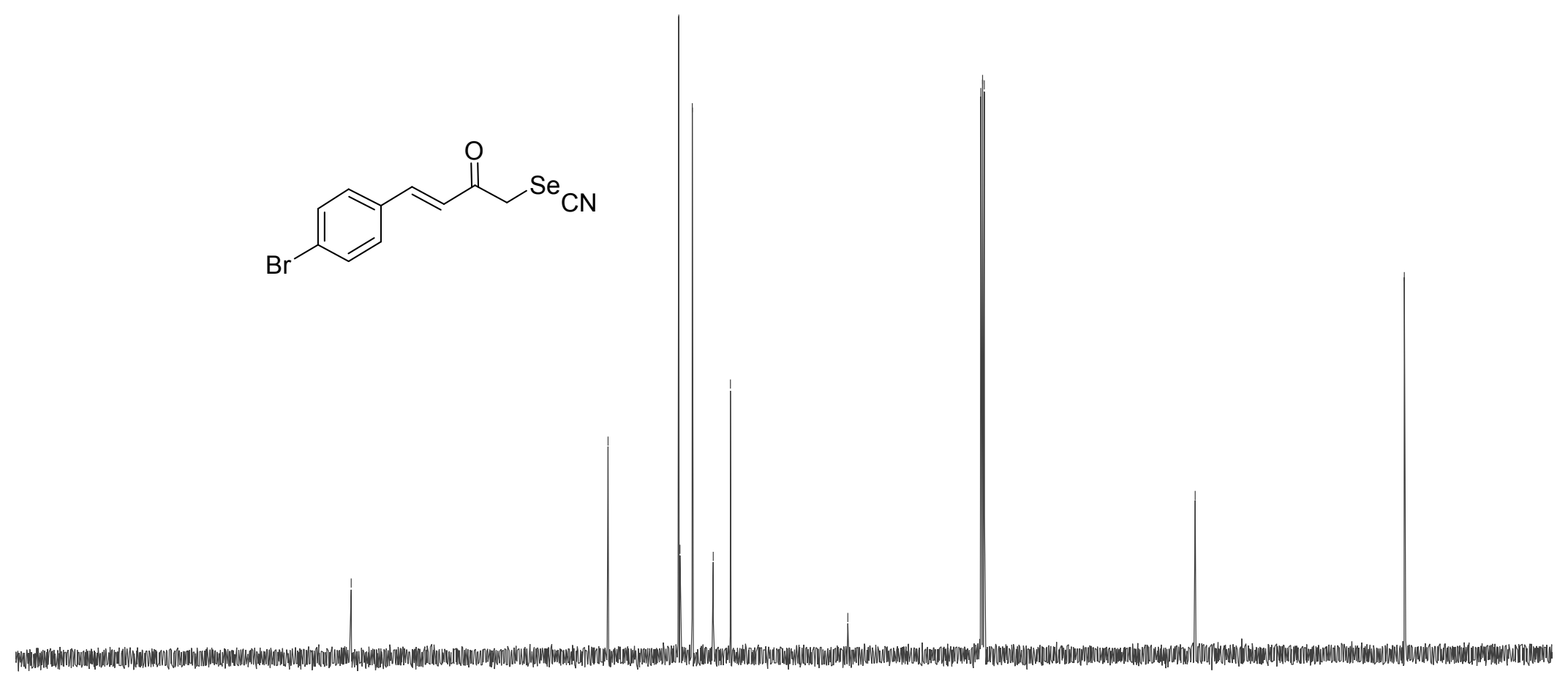

Chemical Shift (ppm)

50

0 
${ }^{1} \mathrm{H}$ NMR (400 MHz, $\mathrm{CDCl}_{3}$ ) spectrum of 4-(4-chlorophenyl)-1-selenocyanatobut-3-en-2-one (5f)

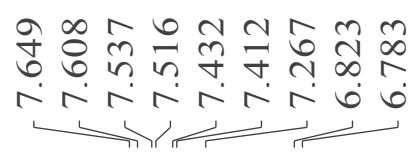

nิ

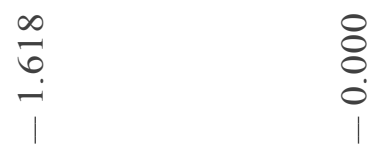
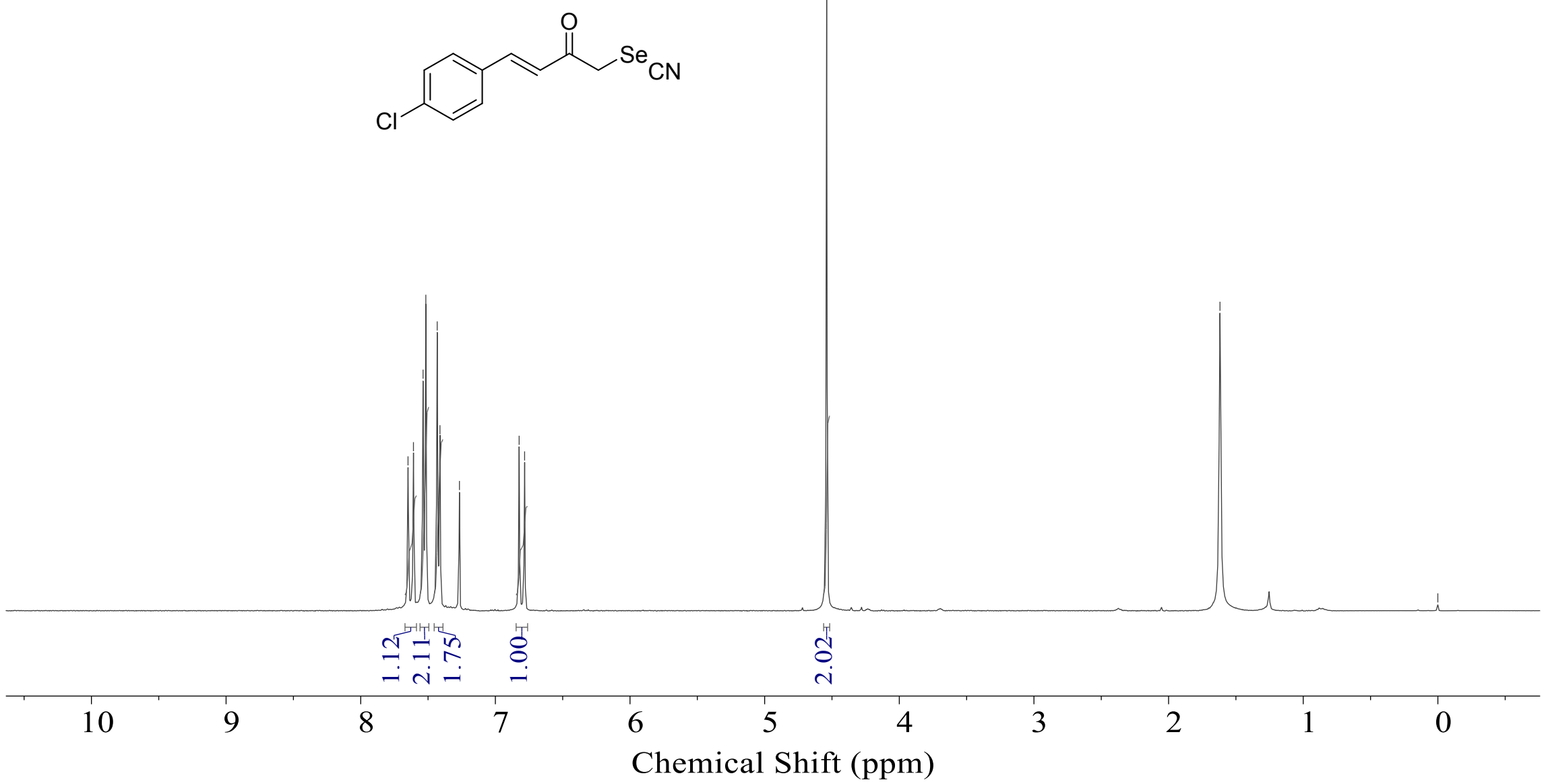
${ }^{13} \mathrm{C}\left\{{ }^{1} \mathrm{H}\right\}$ NMR (100 MHz, $\left.\mathrm{CDCl}_{3}\right)$ spectrum of 4-(4-chlorophenyl)-1-selenocyanatobut-3-en-2-one (5f)

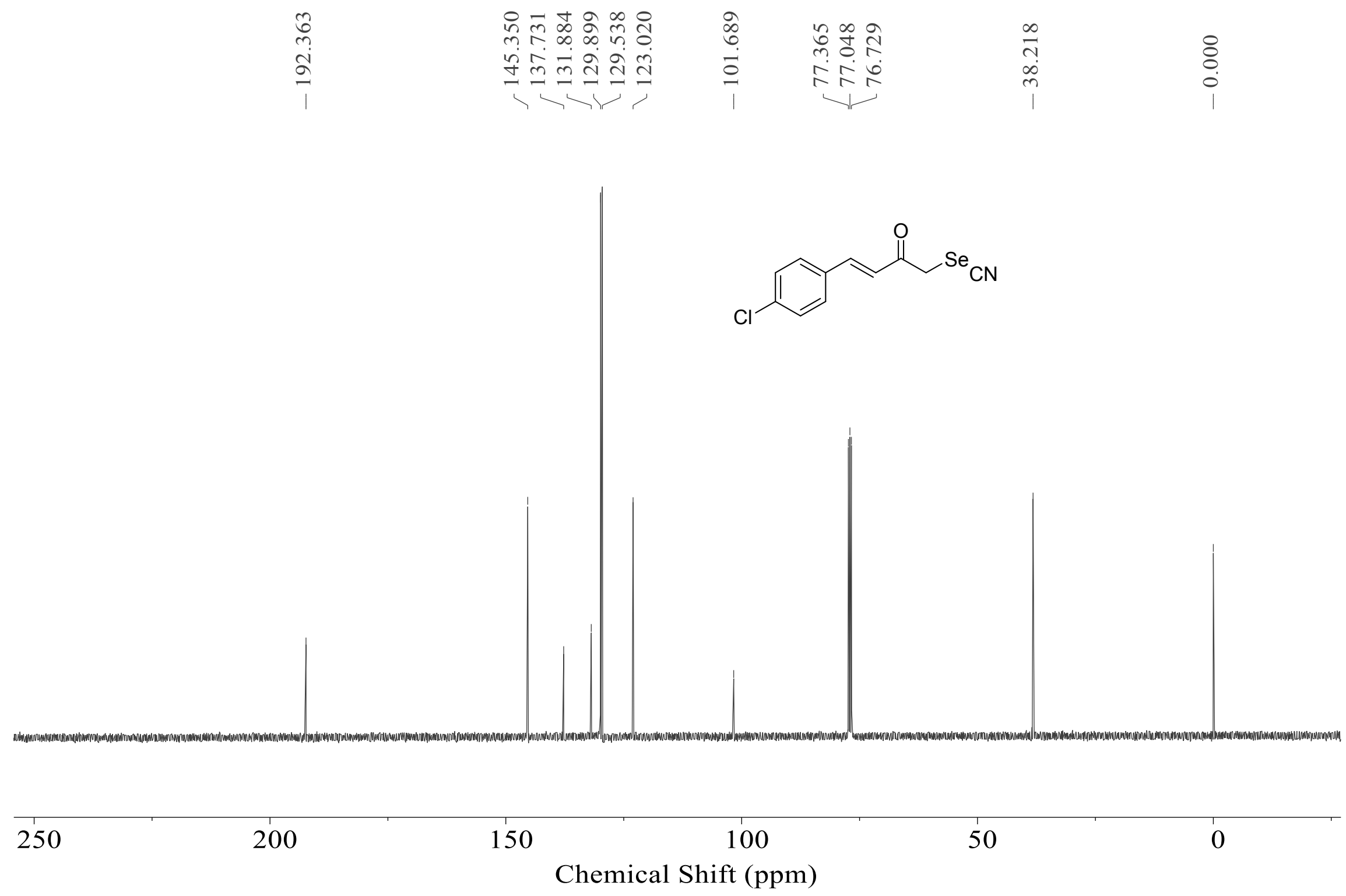


${ }^{1} \mathrm{H}$ NMR (400 MHz, $\mathrm{CDCl}_{3}$ ) spectrum of 4-(2-chlorophenyl)-1-selenocyanatobut-3-en-2-one (5g)

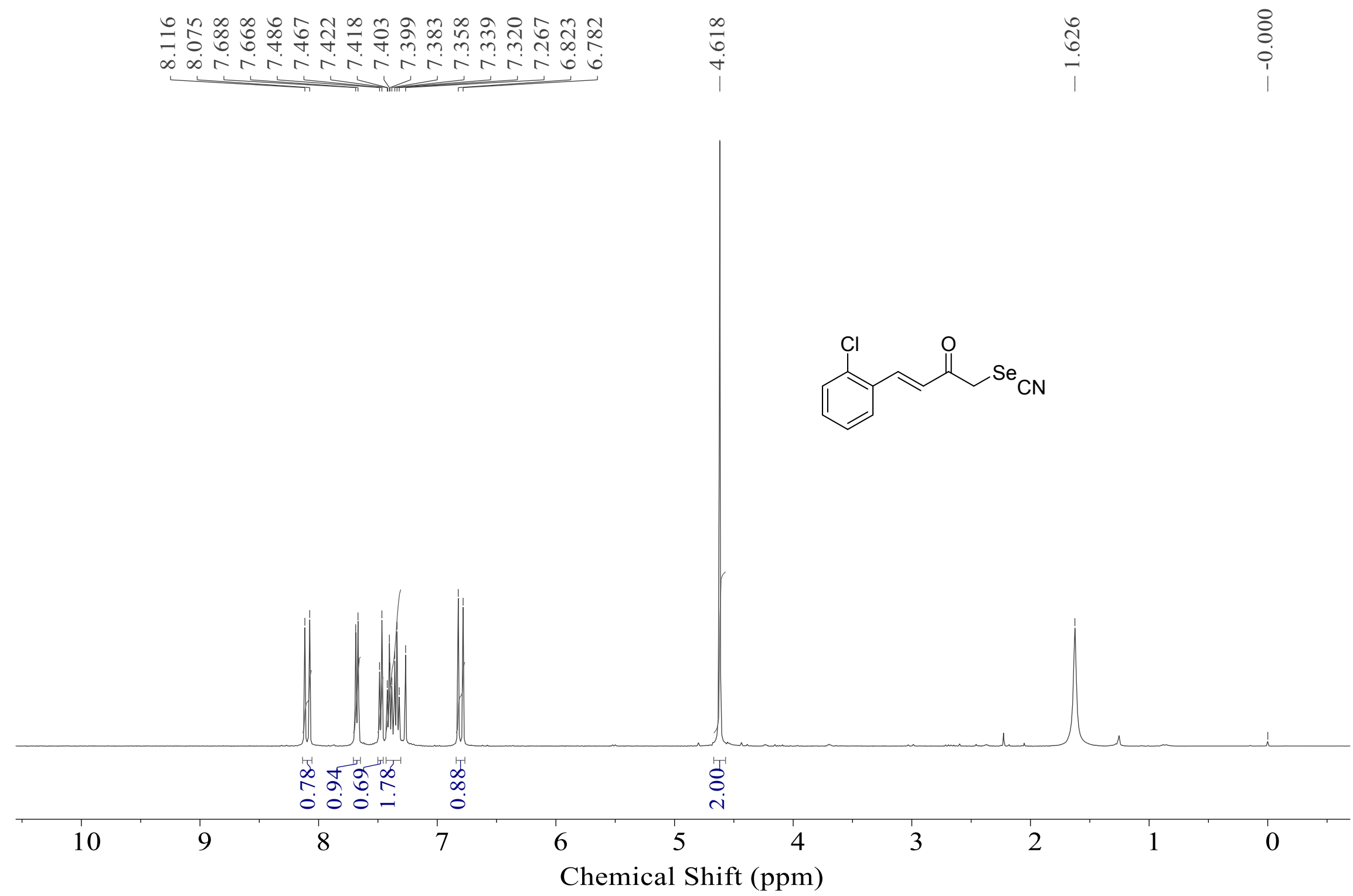


${ }^{13} \mathrm{C}\left\{{ }^{1} \mathrm{H}\right\}$ NMR $\left(100 \mathrm{MHz}, \mathrm{CDCl}_{3}\right)$ of spectrum 4-(2-chlorophenyl)-1-selenocyanatobut-3-en-2-one (5g)

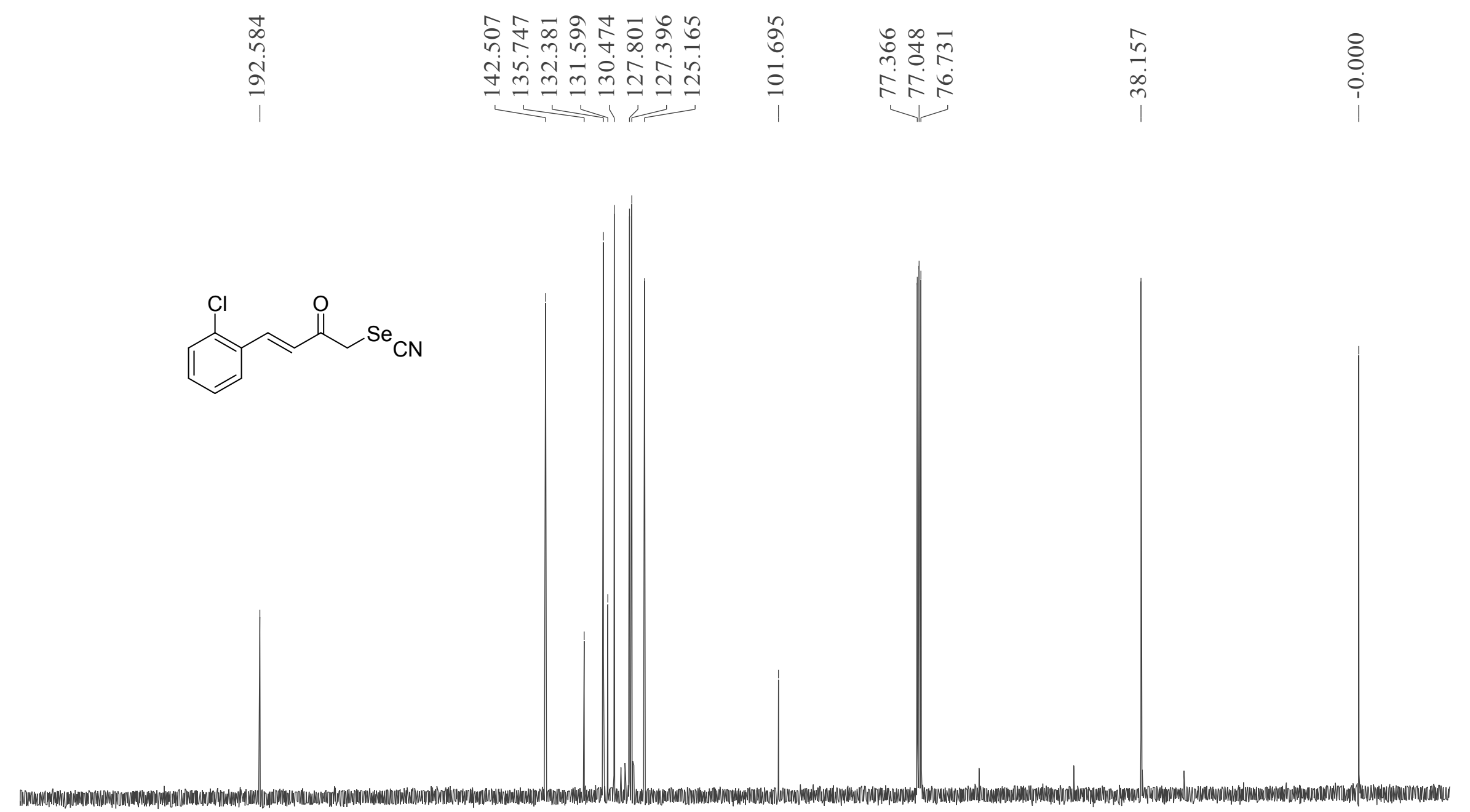


${ }^{1} \mathrm{H}$ NMR (400 MHz, $\mathrm{CDCl}_{3}$ ) spectrum of 4-(2,4-dimethoxyphenyl)-1-selenocyanatobut-3-en-2-one (5h)

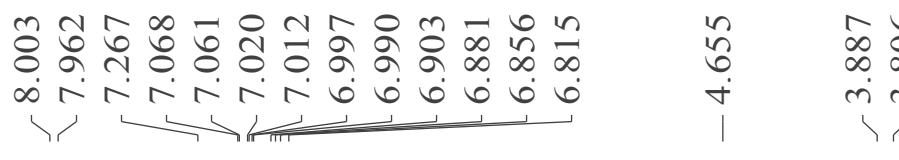

$\begin{array}{ll}n & 8 \\ & \stackrel{0}{0} \\ -1 & \end{array}$
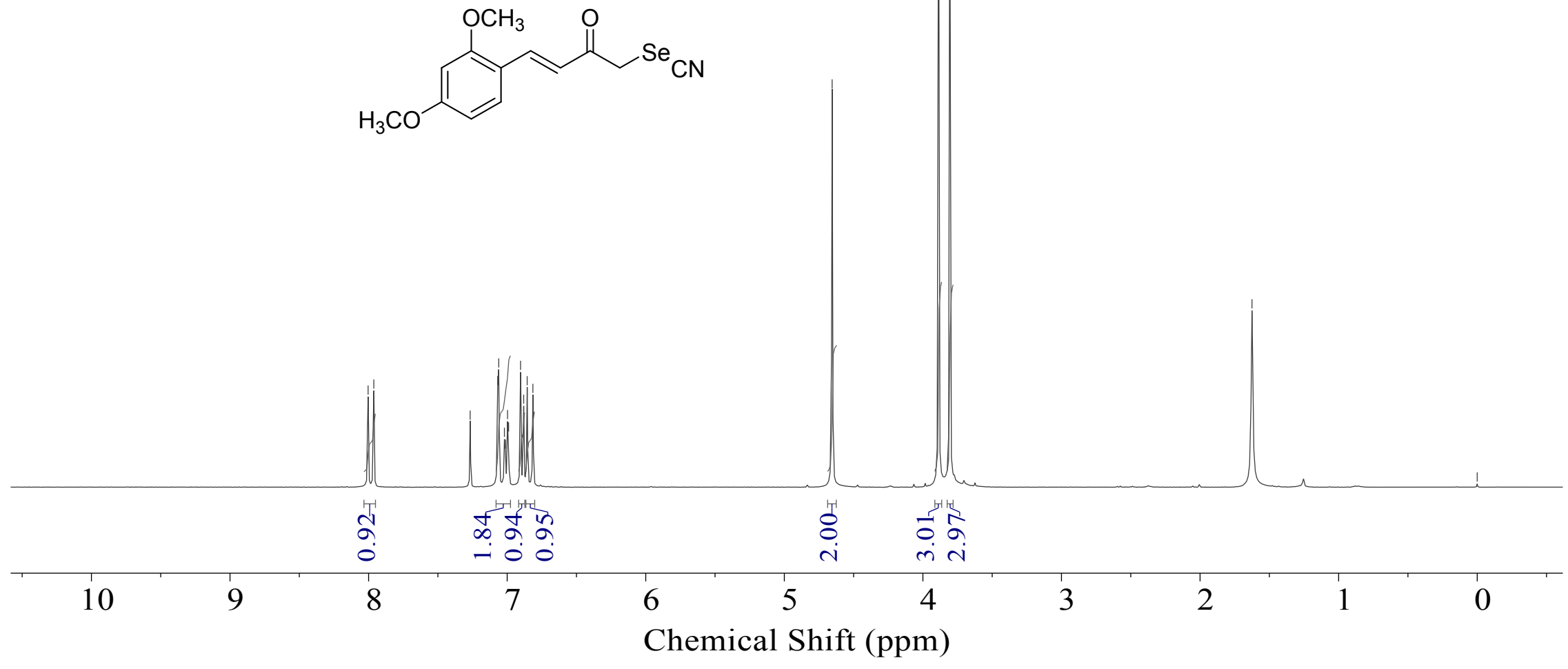
${ }^{13} \mathrm{C}\left\{{ }^{1} \mathrm{H}\right\}$ NMR $\left(100 \mathrm{MHz}, \mathrm{CDCl}_{3}\right)$ spectrum of 4-(2,4-dimethoxyphenyl)-1-selenocyanatobut-3-en-2-one (5h)

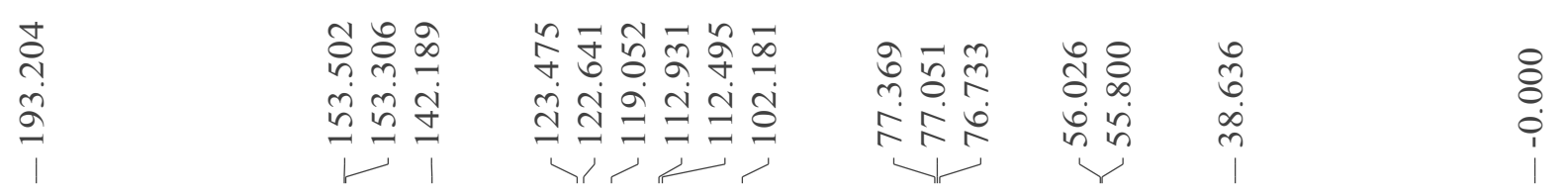<smiles>COc1ccc(/C=C/C(=O)C[Se][Na])c(OC)c1</smiles>

1.

$\begin{array}{ccc}200 & 150 & 100 \\ & & \text { Chemical Shift (ppm) }\end{array}$


${ }^{1} \mathrm{H}$ NMR (400 MHz, $\mathrm{CDCl}_{3}$ ) spectrum of 4-methyl-1-selenocyanatopent-3-en-2-one (5i)

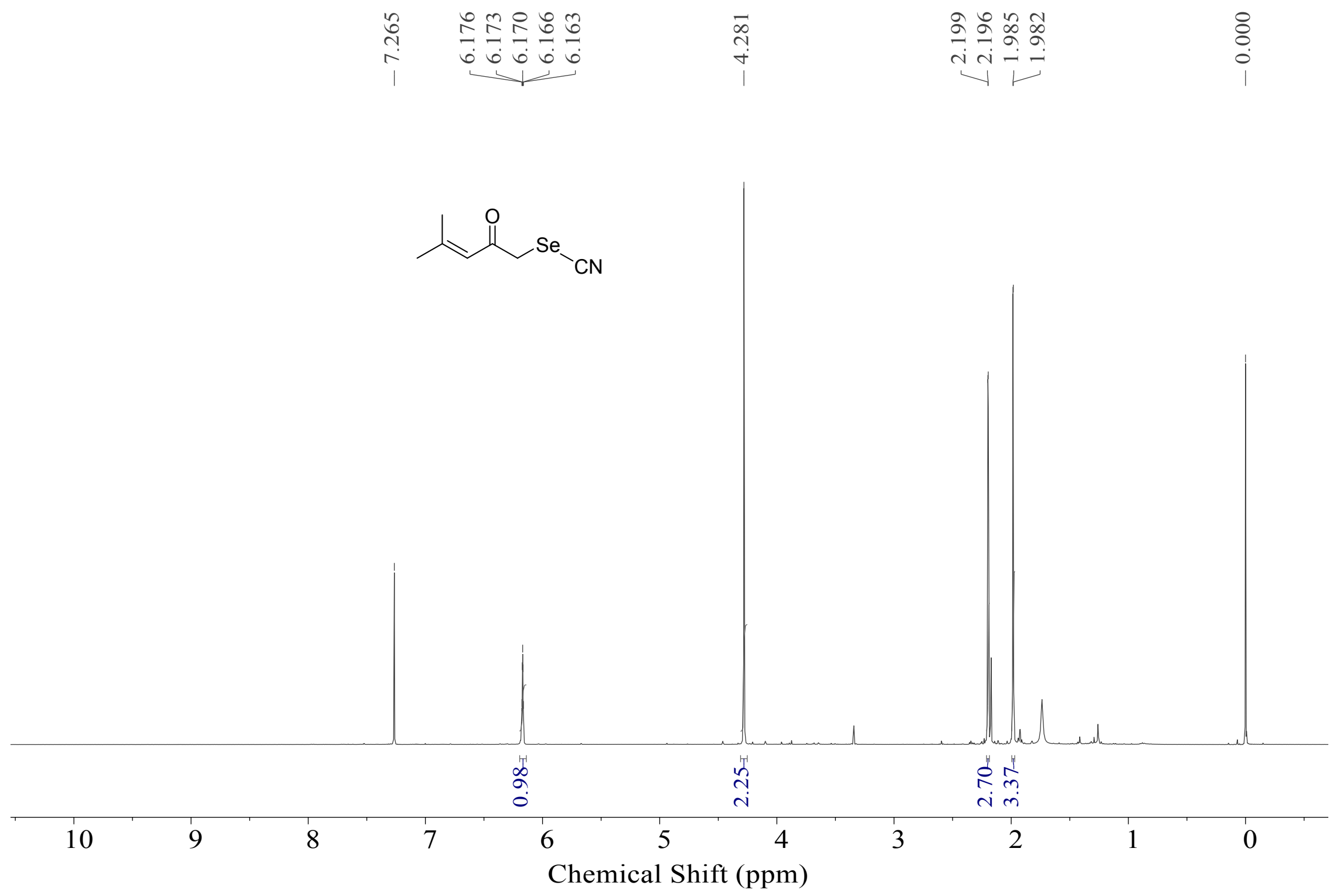


${ }^{13} \mathrm{C}\left\{{ }^{1} \mathrm{H}\right\}$ NMR $\left(100 \mathrm{MHz}, \mathrm{CDCl}_{3}\right)$ spectrum of 4-methyl-1-selenocyanatopent-3-en-2-one (5i)

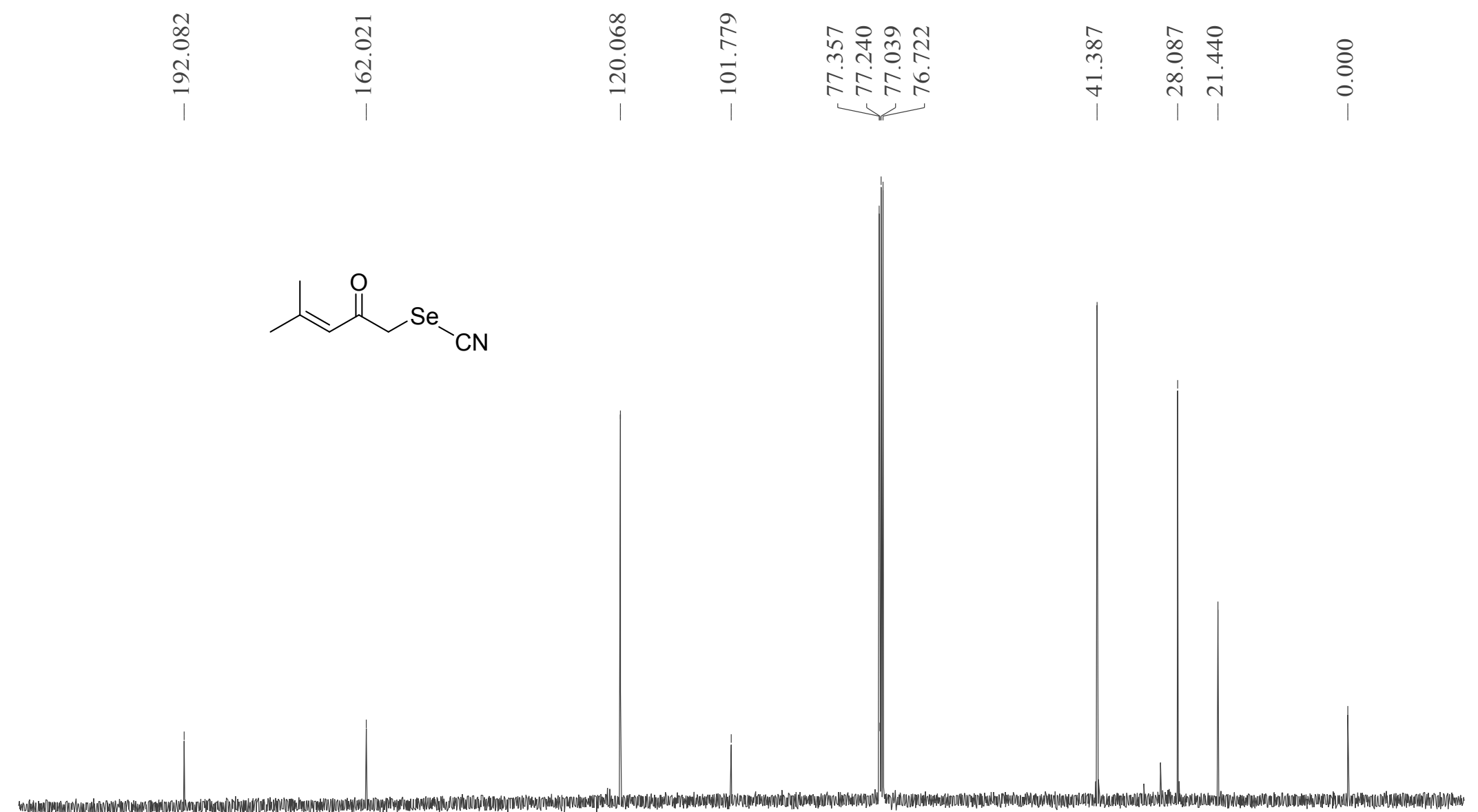

\begin{tabular}{llllllllll}
\hline 200 & 180 & 160 & 140 & $\begin{array}{l}120 \\
\text { Chemical Shift }(\mathrm{ppm})\end{array}$
\end{tabular}

